
\title{
R Research Soure \\ Pattern Analysis of Continuous Analytic Wavelet Transforms of the COVID19 Spreading and Death
}

Yanshuo Wang ( $\sim$ bill.wang@lllw-llc.com )

IIIw Ilc https://orcid.org/0000-0001-6797-3307

\section{Method Article}

Keywords: COVID19, Wavelet, Frequency, Spectrum, Time Series, Scale, Magnitude, Location

Posted Date: May 20th, 2020

DOl: https://doi.org/10.21203/rs.3.rs-29516/v1

License: (9) This work is licensed under a Creative Commons Attribution 4.0 International License. Read Full License

Version of Record: A version of this preprint was published on October 9th, 2020. See the published version at http://dx.doi.org/10.3934/bdia.2020003. 


\title{
Pattern Analysis of Continuous Analytic Wavelet Transforms of the COVID19 Spreading and Death
}

\author{
Yanshuo Wang*, Data Mining and Reliability Consultant
}

*Correspondence to: bill.wang@11lw-1lc.com

Abstract:

Wavelet transform is frequently used for time series data to extract the frequency localization information in last two decades. The wavelet transform with Morse wavelet is applied to the daily infected cases and deaths of COVID19 at different countries/places. There are multiple scales (frequencies) of COVID19 spreading with different magnitude at the specific time which can be identified in Wavelet magnitude scalogram plots. For example, in China, the highest magnitude (2000 to 2500) spreading was happened on 2/12/202 with scale around 329 to 434 , but in U.S.A the highest magnitude (4000 to 4500) spreading was happened during $4 / 25 / 2020$ and 4/28/2020 with scale 137 to 183 . The summary of the wavelet magnitude and scale at specific period for different countries/places is presented in this paper.

Summary:

This paper is to use the wavelet transform method with Morse wavelet to analyze the daily confirmed cases and deaths of COVID in China, U.S.A, Spain, Italy, France, Germany, U.K., South Korea, Michigan and New York City.

\section{Introduction:}

It was announced by the "WHO" that COVID19 was first localized in Wuhan, Hubei Province, China in December, 2019, and it has been a significant human threat to the public health around the globe. As of May 6, 2020: globally, there have been about 3.854 million confirmed case, and about 266,000 reported deaths [3].In U.S.A., there are about 1,266,442 confirm cases, and about 74,948 reported deaths [3].In the state of Michigan, there are about 45,054 confirmed cases and about 4,250 reported death at the time author writing this paper [3][4]. The COVID19 is affecting 210 countries and territories around the world and 2 international conveyances. The COVID19 is spreading into all the 50 states, District of Columbia and its territories in United States. Because of the contagious of this disease, most of the states such as Michigan have issued the staying home order to reduce the infectious speed.

The wavelet transform is frequently used for time series data to extract the frequency localization information in last two decades. It is also used to extract information from epidemiological time series. Numerous studies in epidemiology consist of analyzing time series to extract information and to identify scales of different patterns [1] [9]. The wavelet transform is well adapted to studying non-stationary signals, so we can develop the wavelet transform to extract the information on the COVID19 spreading/death time series data. Wavelet analysis provides both scale and time information of the analyzed signal and allows an objective separation of different structures over different scales at different times. The novel pandemic COVID19 needs more study for researchers to understand its traveling waves and spatial hierarchies. The intention of this paper is attempt to understand the spreading characteristics of COVID19 from a spectrum 40 point of view. The spreading pattern of COVID19 is different from different countries/places. Both government executive orders and culture behavior are playing an important role to slow 
down the spreading of the disease. The wavelet spectrum will give us a fresh view of COVID19 spreading wave and pattern in different places.

The author has used the wavelet analysis for the boundary layer turbulence data in his Master of Science thesis research in 1997 [7-9]. The author believes wavelet transform is a very useful mathematical tool to extract the valuable information from the time series data. That is the reason why the author attempts to use the wavelet analysis to find the spreading or death pattern of COVID19.

\section{Wavelet Transform:}

\section{Continuous Wavelet Transform:}

The wavelet transform of a function $f(t)$ with finite energy is defined as the integral transform with a family of functions $\psi_{s, t}^{*}(u)=\frac{1}{\sqrt{s}} \psi^{*}\left(\frac{u-t}{s}\right)(1)$. It is

$$
W f(s, t)=<f(u), \psi_{\mathrm{s}, \mathrm{t}}>=\int_{-\infty}^{+\infty} f(u) \psi_{\mathrm{s}, \mathrm{t}}^{*}(u) d u=\int_{-\infty}^{+\infty} f(u) \frac{1}{\sqrt{s}} \psi^{*}\left(\frac{u-t}{s}\right) d u
$$

Where $s$ is a scale parameter, $t$ is a location parameter and the function $\psi^{*}{ }_{s, t}(u)=\frac{1}{\sqrt{s}} \psi^{*}\left(\frac{u-t}{s}\right)$ are called wavelet. $\psi^{*}\left(\frac{u-t}{s}\right)$ is called the "mother wavelet". The parameters $s(s \neq 0)$ and $t$ are used to adjust the shape and location of the wavelets respectively.

\section{The Morse Wavelets:}

The generalized Morse wavelets are a family of exactly analytic wavelets. They are useful for analyzing modulated signals, which are signals with time-varying amplitude and frequency [19]. They are also useful for analyzing localized discontinuities, and isolating and extracting quantified features in time-frequency domain. Morse wavelets are defined in the frequency domain as

$\psi_{P, \Upsilon}(\omega)=U(\omega) a_{P, Y} \omega^{\frac{P^{2}}{\Upsilon}} e^{-\omega^{\Upsilon}}$

where $U(\omega)$ is the unit step, $a_{P, Y}$ is a normalizing constant, $P^{2}$ is the time-bandwidth product, and $r>0$ is the shape parameter and characterizes the symmetry of the Morse wavelet. Much of the literature about Morse wavelets uses the oscillation control parameter $\beta>0$, which can be viewed as a decay or compactness parameter, rather than the time-bandwidth product, $P^{2}=\beta r$. The equation for the Morse wavelet in the Fourier domain parameterized by $\beta$ and $\gamma$ is as following:

$\psi_{\beta, r}(\omega)=\int_{-\infty}^{\infty} \psi_{\beta, \Upsilon}(t) e^{-i w t} d t=U(\omega) a_{\beta, \Upsilon} \omega^{\beta} e^{-\omega^{\Upsilon}}$

where $a_{\beta, \Upsilon} \triangleq 2\left(\frac{e \Upsilon}{\beta}\right)^{\frac{\beta}{\gamma}}$

(5) is a normalization constant ensuring a peak frequency of 2 [11-17]. The effect of parameter $Y$ and $P^{2}$ values on Morse Wavelet shape are shown in Fig 1. This figure is borrowed from Matlab Morse Wavelet Tutorial [19].

\section{Application of Wavelet Transform:}

Wavelet variance (also called wavelet spectrum): Wavelet variances are computed by integrating fluctuations of wavelet functions over time for every given time scale. The values of the wavelet variance indicate the relative intensities of spreading structures at different scales. 
Thus the wavelet variance may be useful for providing information about the time-scales of COVID19 spreading processes. Given the interpretation of the wavelet variance in terms of COVID19 spreading energy distribution, the scales corresponding to special peaks must contribute most to spreading energy.

Wavelet decomposition: The Mallat's multiresolution algorithm [18] can be used to retrieve the original from the whole set of wavelet coefficients. This "reconstruction" enables one to filter the original function, by splitting the integration domain into specific ranges of scales. Wavelet filtering could isolate the two phenomena in COVID19 spreading data, if they have different scales. The wavelet decomposition is not in the scope of this paper, but it can be done in future study of COVID19.

Jump detection: "Jump" means a sharp increase or decrease in a signal. Wavelet is a localized transform, so it is useful for jump detection. COVID19 may be associated with sudden changes or apparent discontinuities in data time series. The wavelet transform can provide information about these sudden changes and apparent discontinuities on different scales.

Time-Frequency Representation: Wavelets have been considered from both time and frequency points of view. For scientific applications, the localization in time and frequency domains is a key property in signal analysis. From the time-frequency plot for COVID19 spreading, the COVID19 spreading can be recognized at different scales with the passage of time. Time-frequency representation demonstrates the usefulness of wavelet analysis in decomposing spreading waves at different scales hidden in time series data. Using twodimensional unfolding in both time and scale, the techniques provides the location and localized spectrum for COVID19 spreading of interest. This method is especially useful for studying short lived transient events of COVID19.

\section{Wavelet Analysis:}

In China:

The figure 2 shows the daily infected cases and deaths in China. From 1/23/2020 (day 2) to $2 / 4 / 2020$ (day 14), the trend of the daily infected case in China is increasing, although there is small peak on $1 / 27 / 2020$ (day 6). From 2/4/2020 (day 14) to 2/11/2020 (day 21), the infectious trend is decreasing. There is a big jump on 2/12/2020 (day 22), the infected cases are up to 14,108. After this huge jump, the trend of the daily infected case is going down until 2/19/2020 (day 29). Then the trend of the daily infected cases is up and down until it is almost diminished. The Morse wavelet transform was applied to China daily infected cases and deaths. The magnitude scalogram is shown in figure 3 and figure 4 . The horizontal axis is represented the days when COVID19 spreading. The vertical axis is represented the scale or frequency of the infection or death. The color represents the magnitude or energy of the infection or death. From the figure 3, there is a strong magnitude of the infection around day $22(2 / 12 / 2020)$, and the scale is about from 329 to 434 for high magnitude (about 2000 to 2500) infection, in the meantime, the scale is from 183 to 329 and the magnitude is about 1500 to 2000 spreading as well. The wavelet scalogram is shown the different magnitude of COVID19 spreading with different scales at the specific time period. Overall, the COVID19 spreading in China is shown the highest speak at the maximum 434 scale around day $22(2 / 12 / 2020)$, the spreading energy is decreasing after about day $50(3 / 11 / 2020)$. Between day $30(2 / 20 / 2020)$ to day $50(3 / 11 / 2020)$, the magnitude (around $1000)$ is more concentrating on the scale from 206 to 30. After day $50(3 / 11 / 2020)$ the spreading magnitude (dark blue color in plot) is really decreasing. This can correlate the government 
isolation policy in China. The author believe we can learn the COVID19 spreading trend at the specific days on different spreading frequency/scale with different spreading magnitude/energy from the wavelet analysis.

But looking at the magnitude scalogram of death in China from figure 4 , the death peak (the magnitude about 320) is around day $87(4 / 17 / 2020)$ at the scale from 329 to 434. Between day 83 $(4 / 13 / 2020)$ to day $90(4 / 20 / 2020)$, the medium magnitude is from 200 to 250 with the scale from 206 to 329. The wavelet is an excellent tool to extract the COVID 19 spreading and deaths magnitude and scale patterns at different period. This may help us to understand the spreading and death nature of COVID19 and the effectiveness of the government policy.

\section{In U.S.A.:}

The Morse wavelet analysis is also applied to U.S.A daily infected cases and deaths. They are shown in figure 6 and figure 7. From U.S.A spreading magnitude scalogram plot, the highest peak (magnitude about 4000 to 4500 ) is happened around the scale from 137 to 183 at the day 71 $(4 / 25 / 2020)$ to day $74(4 / 28 / 2020)$. It seems after day $40(3 / 25 / 2020)$, the scale around 149 is the major threat which has the medium magnitude which changed to highest magnitude on day 71 (4/25/2020), and also the scale around 329 with magnitude about 3000 is also the threat at day 78 $(5 / 2 / 2020)$.

From the death magnitude scalogram (figure 7), the highest magnitude (500 to 550) of death is happened around the scale 137 to 164 at day $73(4 / 27 / 2020)$ to day 75 (4/29/2020). After day 50 $(4 / 4 / 2020)$ the scale around 150 is the major threat, and the scale around 274 at day 78 $(5 / 2 / 2020)$ with magnitude (350 to 400$)$ is also the threat.

\section{In Spain:}

The Morse wavelet analysis is also applied to Spain daily infected cases and deaths. They are shown in figure 9 and figure 10. The highest spreading peak (magnitude about 900 to 1070) is happened around the scale from 137 to 164 on day $53(4 / 7 / 2020)$ to day $73(4 / 27 / 2020)$. There is another peak around scale 412 with magnitude about 900 to 1070 at day 60 (4/14/2020) to day $70(4 / 24 / 2020)$. The medium spreading magnitude (around 600 to 700 ) is happened from day 35 $(3 / 20 / 2020)$ to day $77(5 / 1 / 2020)$ with different scales.

From the death magnitude scalogram (figure 10), the highest magnitude (70 to 80 ) of death is happened around the scale 274 to 411 on day 57 (4/11/2020) to day 65 (4/19/2020). Around day $40(3 / 25 / 2020)$, the scale around 42 also has the high magnitude (70 to 80$)$. After day 30 $(3 / 15 / 2020)$, the medium magnitude around 50 is also the threat with different scale.

\section{In Italy:}

The Morse wavelet analysis is also applied to Italy daily infected cases and deaths. They are shown in figure 12 and figure 13. The highest spreading peak (magnitude about 600 to 700 ) is happened around the scale from 126 to 164 on day $32(3 / 17 / 2020)$ to day $55(4 / 9 / 2020)$. The medium spreading magnitude (around 400) is happened from day 27 (3/12/2020) to day 72 (4/26/2020) with different scales.

From the death magnitude scalogram (figure 13), the highest magnitude ( 75 to 85 ) of death is happened around the scale 274 to 329 on day $35(3 / 20 / 2020)$ to day $45(3 / 30 / 2020)$. Around day $72(4 / 26 / 2020)$ to day $77(5 / 1 / 2020)$, the scale 235 to 274 also have the highest magnitude (75 to 
85). After day $30(3 / 15 / 2020)$, the medium magnitude from 60 to 70 is the threat with different scale.

\section{In France:}

The Morse wavelet analysis is also applied to France daily infected cases and deaths. They are shown in figure 15 and figure 16. The highest spreading peak (magnitude 3000 to 3500 ) is happened around the scale from 235 to 412 on day $47(4 / 1 / 2020)$ to day $53(4 / 7 / 2020)$. The medium spreading magnitude (2000 to 2500$)$ is happened from day $42(3 / 27 / 2020)$ to day 56 $(4 / 10 / 2020)$ with different scales.

From the death magnitude scalogram (figure 16), the highest magnitude (280 to 320) of death is happened around the scale 329 to 412 on day 49 (4/3/2020) to day 55 (4/9/2020). After day 45 (3/30/2020), the medium magnitude 200 to 250 is also the threat with different scale.

\section{In Germany:}

The Morse wavelet analysis is also applied to Germany daily infected cases and deaths. They are shown in figure 18 and figure 19. The highest spreading peak (magnitude about 1000 to 1100) is happened around the scale from 137 to 164 on day $38(3 / 23 / 2020)$ to day $58(4 / 12 / 2020)$. The medium spreading magnitude (750 to 900) is happened after day 32 (3/17/2020) with different scales.

From the death magnitude scalogram (figure 19), the highest magnitude (60 to 65) of death is happened around the scale 137 to 149 on day 53 (4/7/2020) to day 72 (4/26/2020). Around day $79(5 / 3 / 2020)$, the scale 412 also have the strong magnitude (around 60). After day 47 (4/1/2020), the medium magnitude from 40 to 60 is also the threat with different scale.

\section{In U.K.:}

The Morse wavelet analysis is also applied to U.K. daily infected cases and deaths. They are shown in figure 21 and figure 22. The highest spreading peak (magnitude about 1200 to 1300) is happened around the scale around 412 on day $50(4 / 4 / 2020)$ to $54(4 / 8 / 2020)$. The medium spreading magnitude (around 800 to 1100$)$ is happened after day $46(3 / 31 / 2020)$ with different scales.

From the death magnitude scalogram (figure 22), the highest magnitude (200 to 250) of death is happened around the scale 273 to 329 on day $63(4 / 17 / 2020)$ to day $74(4 / 28 / 2020)$. There is a strong medium magnitude (150 to 200) is happened after day 50 (4/4/2020) with different scales.

\section{In South Korea:}

The Morse wavelet analysis is also applied to South Korea daily infected cases and deaths. They are shown in figure 24 and figure 25. The highest spreading peak (magnitude about 140 to 150) is happened around the scale 412 on day $14(2 / 28 / 2020)$ to day $19(3 / 3 / 2020)$.

From the death magnitude scalogram (figure 25), the highest magnitude (around 3.5) of death is happened around the scale 412 on day $20(3 / 4 / 2020)$ to day $31(3 / 16 / 2020)$.

\section{In Michigan:}

The Morse wavelet analysis is also applied to Michigan daily infected cases and deaths. They are shown in figure 27 and figure 28. The highest spreading peak (magnitude about 250 to 275) is happened around the scale from 127 to 160 on day $36(4 / 14 / 2020)$ to day $47(4 / 25 / 2020)$. The 
medium spreading magnitude (around 150 to 200) is happened after day 22 (3/31/2020) with different scales.

From the death magnitude scalogram (figure 28), the highest magnitude (45 to 50) of death is happened around the scale 412 on day $42(4 / 20 / 2020)$ to day $51(4 / 29 / 2020)$. There is a strong medium magnitude (35 to 40 ) with scale 235 to 325 is also happened on day 42 (4/20/2020) to day $51(4 / 29 / 2020)$. After day $30(4 / 8 / 2020)$, the medium magnitude around 30 is also the threat with different scale.

\section{In New York City:}

The Morse wavelet analysis is also applied to New York daily infected cases and deaths. They are shown in figure 30 and figure 31 . The highest spreading peak (magnitude 700 to 800 ) is happened around the scale from 137 to 164 on day $12(3 / 20 / 2020)$ to day $46(4 / 23 / 2020)$. There is another peak around scale 236 to 277 with magnitude around 700 to 800 at day $16(3 / 24 / 2020)$ to day $32(4 / 9 / 2020)$. New York City has the longest spreading span with high magnitude. Form day $10(3 / 18 / 2020)$ to day $46(4 / 23 / 2020)$ the medium magnitude 500 to 650 are spreading in different scales.

From the death magnitude scalogram (figure 31), the highest magnitude (18 to 22) of death is happened around the scale 137 to 183 on day $24(4 / 1 / 2020)$ to day $40(4 / 17 / 2020)$. After day 20 $(3 / 28 / 2020)$, the medium magnitude around 14 to 18 is also the threat with different scale.

\section{Discussion:}

From the wavelet analysis above for all the places, the wavelet variance (spectrum) of COVID19 spreading and deaths can be easily seen by magnitude scalogram plot. It shows not only the magnitude of the spreading and death, but also the scale (frequency) of the spreading and death at specific time period. The policy makers may use the wavelet spectrum to decide the containment strategies. Also comparing the magnitude scalogram between different places, we may learn the effectiveness of the COVID19 containment.

U.S.A has the highest spreading and death magnitude comparing to other countries. China had the high magnitude at the earlier stage of the spreading as well as South Korea, but South Korea magnitude is much smaller, the Europe countries and U.S.A. had the high magnitude spreading at the later stage. New York City had the longest spreading span period with high magnitude. The spreading scale of the wave are different from different places, for example, at the peak magnitude, China has the scale 329 to 434; U.S.A has the scale 137 to 183; Spain, Germany and New York City have the same spreading scale which is 137 to 164; U.K., Spain and South Korea also have the same scale 412; Italy and Michigan have the similar scale; France and New York City have the similar scale.

COVID19 is traveled and spreading as different scale (frequency) waves, we need learn this from wavelet spectrum to see which scale of wave play the major role. The summary of wavelet analysis is in table 1.

Also as we mentioned in section 2, the jumps of the spreading and death can be easily identified from the colorful plots of magnitude scalogram.

40 In this paper, the generalize Morse wavelet is used for the analysis because it is good for the modulated signals, but it is worthwhile to apply the other orthogonal wavelets such as 
Daubechies in the study, and the wavelet multiresolution analysis should be explored in future study.

\section{Conclusion:}

The aim of this paper is to characterize the spreading and death waves for COVID19 by using the Morse wavelet analysis. The wavelet magnitude scalogram is the most simple and colorful way to explore the scale (frequency) and magnitude (energy) at the specific time period. The wavelet analysis can extract the useful information for the COVID19 spreading and death from time series data. By using the wavelet transform, the author hope to bring a novel view to study the COVID19 spreading and death, and help the public health professionals to concentrate the scale which has the high magnitude of spreading and death. Also this analysis can help the policy makers learn from their counterparts, and make a wise decision to help the public to fight COVID19 in a more effective ways in future.

\section{References and Notes:}

1. Cazelles B, Cazelles K, Chavez M. 2014, "Wavelet analysis in ecology and epidemiology: impact of statistical tests". J. R. Soc. Interface 11: 20130585. http://dx.doi.org/10.1098/rsif.2013.0585

2. Chui, C. K: 1992. "An Introduction to Wavelets", Academic Press, 264pp.

3. WorldOMeters: https://www.worldometers.info/coronavirus/country/us/

4. Click On Detroit News: https://www.clickondetroit.com/news/local/2020/03/20/michigan-covid-19-datatracking-case-count-cases-by-county-deaths-cases-by-age-tests/

5. Liu, Chun-Lin: February, 2010, "A Tutorial of the Wavelet Transform”, 71pp.

6. New York City Gov. https://www1.nyc.gov/site/doh/covid/covid-19-data.page

7. Wang, Yanshuo, "Coupling of Small Scale Turbulence near the Ground to Large Eddy Structures in a Desert Boundary-Layer". 1997. University of Connecticut. Master of Science Thesis

8. David R. Miller, Yanshuo Wang, and Ronald Cionco, "Ground Level Gust Frequency Coupled to PBL Large Eddies", Proceeding of 12th Symposium on Boundary Layers and Turbulence, University of British Columbia, Vancouver, BC, Canada, July, 1997

9. David R. Miller, Yanshuo Wang, and Ronald Cionco, "Detecting of PBL Forcing of Ground Level Winds in a Desert Using Wavelet Analysis Methods". DTIC ADA344056: Proceedings of the 1997 Battlespace Atmospherics Conference 2-4 December 1997

10. Grenfell BT, Bjørnstad ON, Kappey J. 2001, "Travelling waves and spatial hierarchies in measles epidemics". Nature 414, 716-723. (doi:10.1038/414716a)

11. Olhede, S. C., and A. T. Walden. "Generalized morse wavelets." IEEE Transactions on Signal Processing, Vol. 50, No. 11, 2002, pp. 2661-2670.

12. Lilly, J. M., and S. C. Olhede. "Higher-order properties of analytic wavelets." IEEE Transactions on Signal Processing, Vol. 57, No. 1, 2009, pp. 146-160.

13. Lilly, J. M., and S. C. Olhede. "On the analytic wavelet transform." IEEE Transactions on Information Theory, Vol. 56, No. 8, 2010, pp. 4135-4156.

14. Lilly, J. M., and S. C. Olhede. "Generalized Morse wavelets as a superfamily of analytic wavelets." IEEE Transactions on Signal Processing Vol. 60, No. 11, 2012, pp. 6036-6041.

15. Lilly, J. M. jLab: A data analysis package for Matlab, version 1.6.2., 2016. http://www.jmlilly.net/jmlsoft.html.

16. Lilly, J. M. "Element analysis: a wavelet-based method for analysing time-localized events in noisy time series." Proceedings of the Royal Society A. Volume 473: 20160776, 2017, pp. 1-28. dx.doi.org/10.1098/rspa.2016.0776.

17. M. P. Wachowiak, R. Wachowiak-Smolíková, M. J. Johnson, D. C. Hay, K. E. Power, F. M. Williams-Bell, "Quantitative feature analysis of continuous analytic wavelet transforms of electrocardiography and electromyography”, Philos Trans A Math Phys Eng Sci. 2018 Aug 13; 376(2126): 20170250. Published online 2018 Jul 9. doi: 10.1098/rsta.2017.0250

18. Mallat, Stephane, 1989, "A Theory for Multiresolution Signal Decomposition: The Wavelet Representation". IEEE Transactions on Pattern Analysis and Machine Intelligent, 11, 674-693

19. Mathlab wavelet help tutorial. MathWorks 
20. Yanshuo Wang, "Use NHPP (Non-Homogenous Poisson Process) Crow-AMSAA method to model the spreading and death rate of the COVID 19", presented in the SCIENTIFIC PERSPECTIVESON COVID19 conference on May 4th, 2020, Scientific Online Conference.

21. Yanshuo Wang, "Use Crow-AMSAA a method to predict the cases of the Coronavirus 19 in Michigan and U.S.A", medRxiv preprint, doi: https://doi.org/10.1101/2020.04.03.20052845. Submitted to Infectious Disease Modeling Journal under review.

\section{Acknowledgments:}

The author appreciates the data which provided by website in reference [3]. [4] and [6]. The thanks the MathWorks company to provide the Wavelet analysis in Matlab package.

Competing interests: The authors declare no competing interests. 
Supplementary Materials:
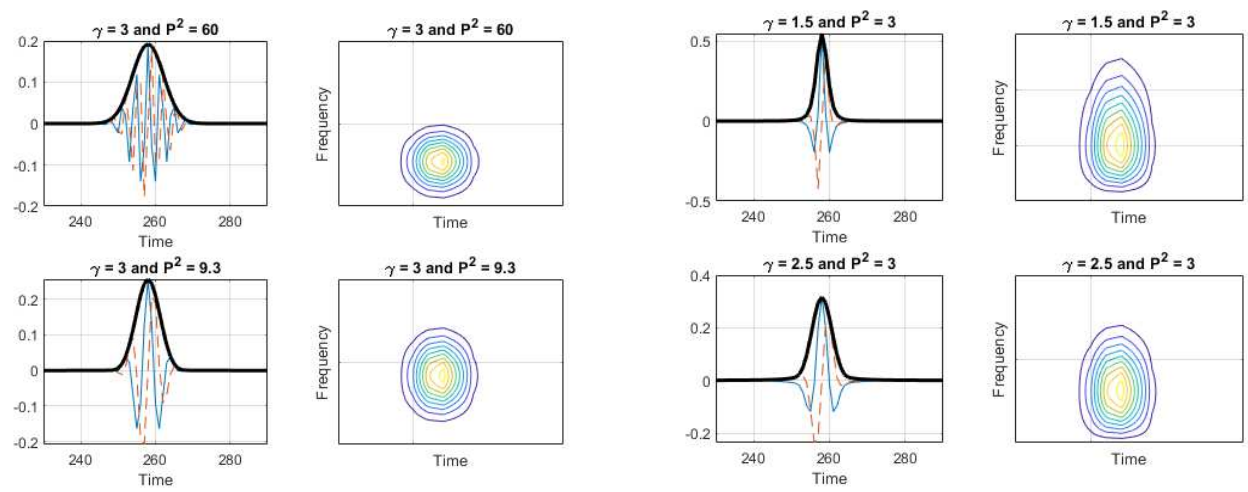

Figure 1: The effect of parameter $Y$ and $P^{2}$ values on Morse Wavelet shape. This figure is from Matlab.

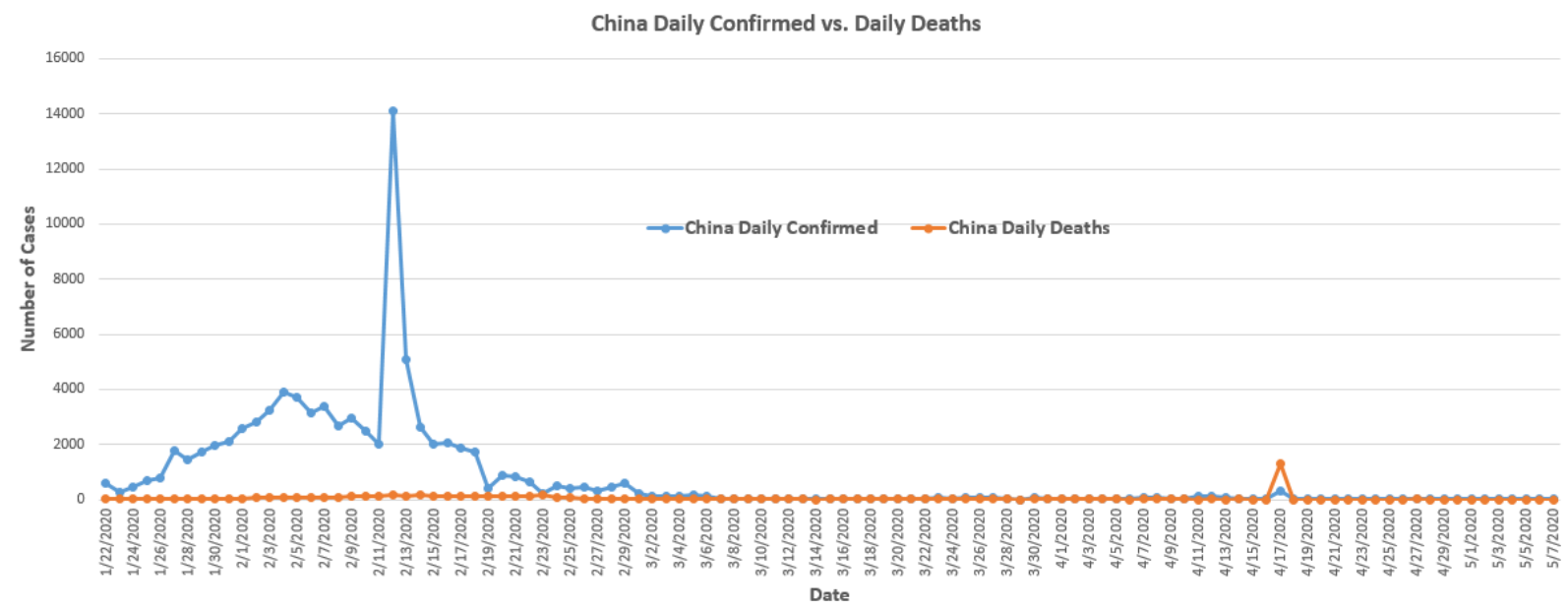

Figure 2: COVID19 - China Daily Infected Cases and Deaths, the date is from 1/22/2020 to 5/7/2020. 


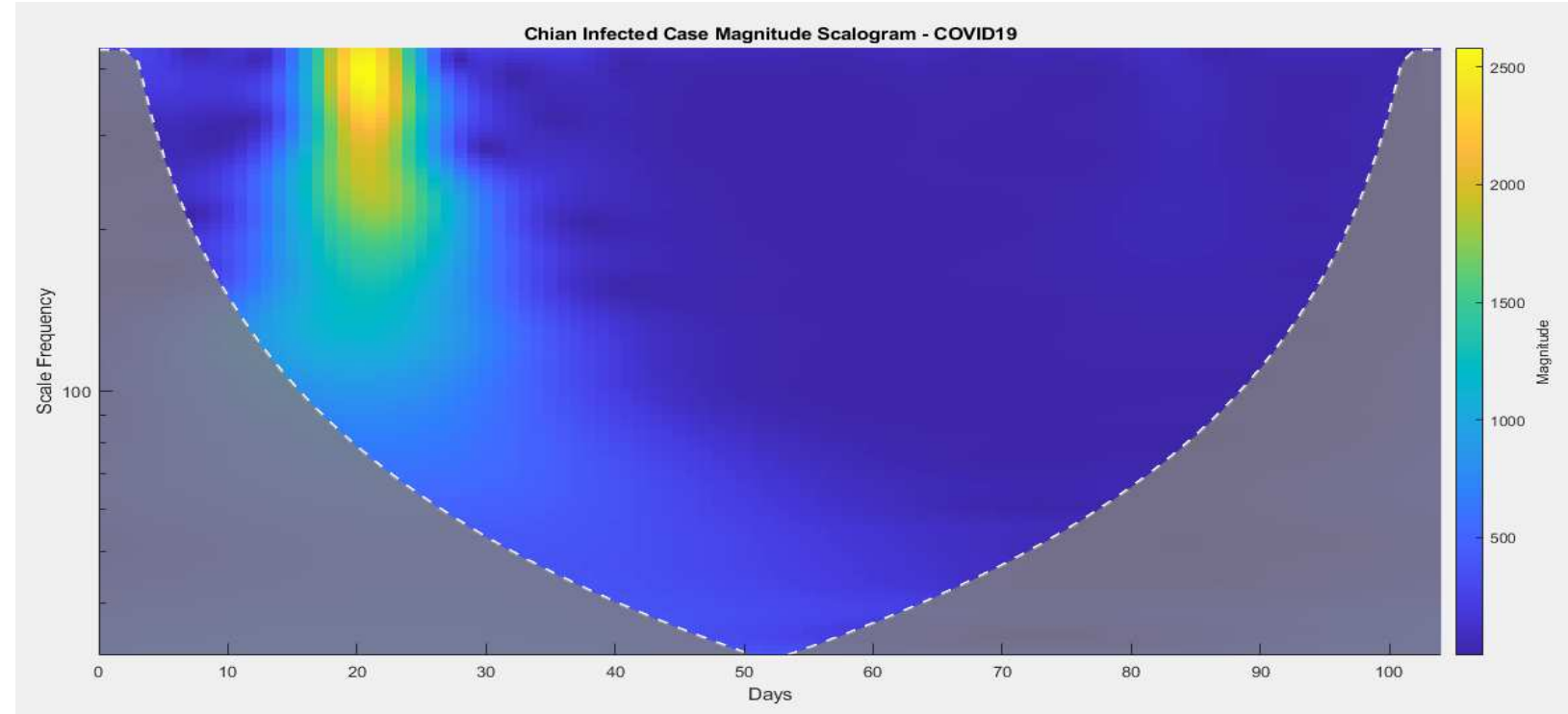

Figure 3: COVID19 - China Daily Infected Cases Wavelet Magnitude Scalogram, the date is from $1 / 22 / 2020$ to $5 / 7 / 2020$.

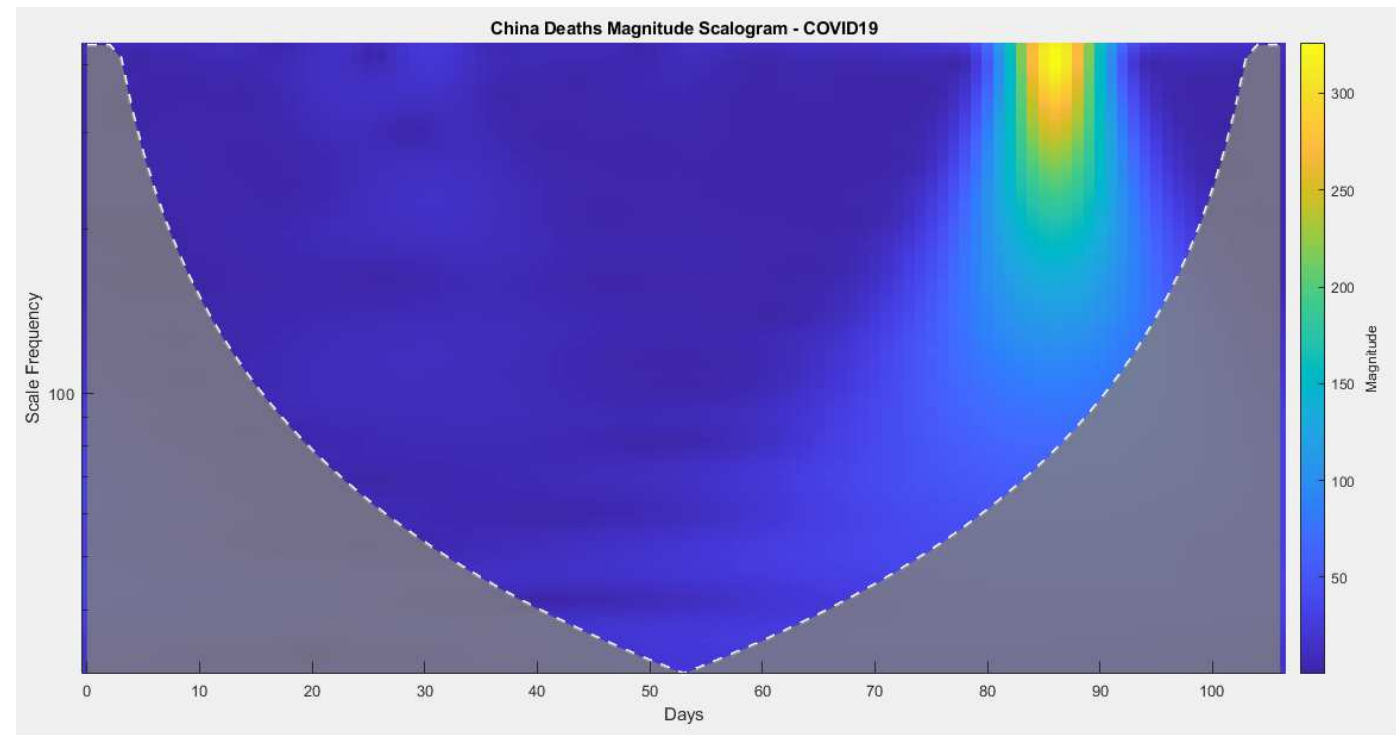

Figure 4: COVID19 - China Daily Deaths Wavelet Magnitude Scalogram, the date is from $1 / 22 / 2020$ to $5 / 7 / 2020$. 


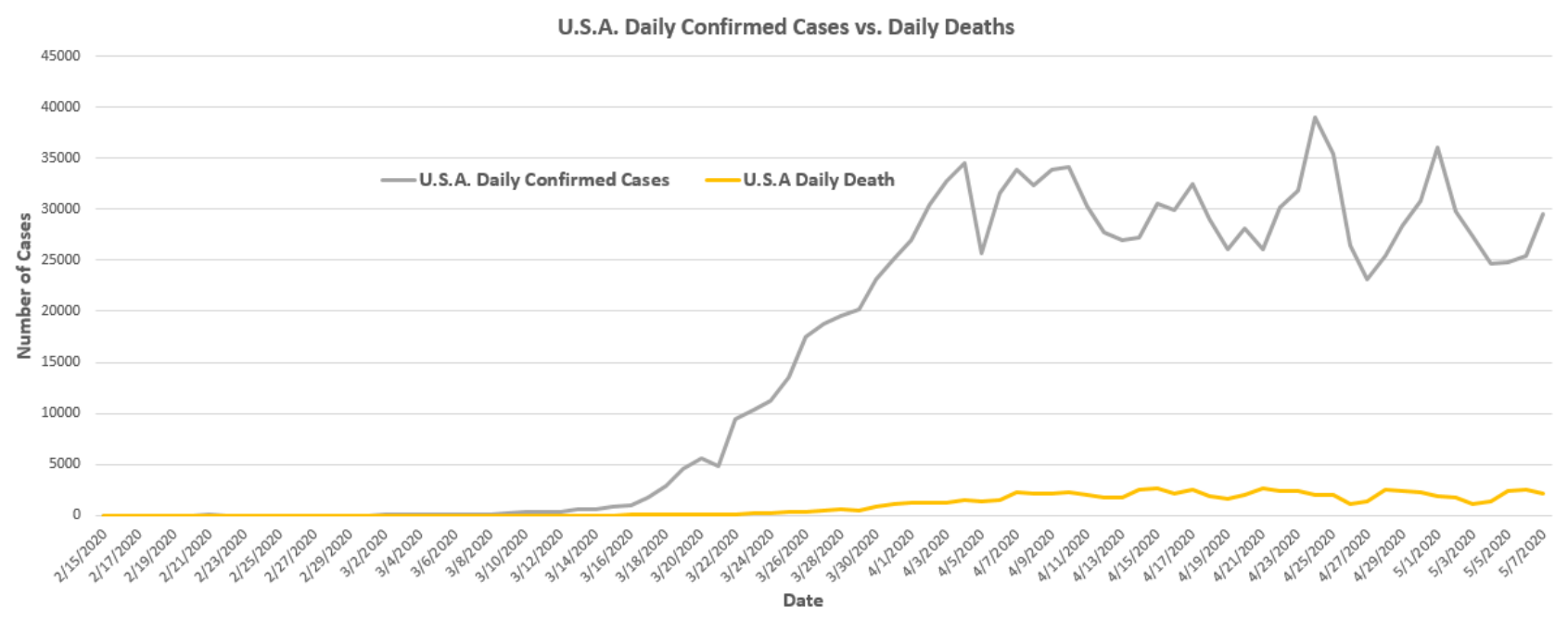

Figure 5: COVID19 - U.S.A Daily Infected Cases and Deaths, the date is from 2/15/2020 to 5/7/2020.

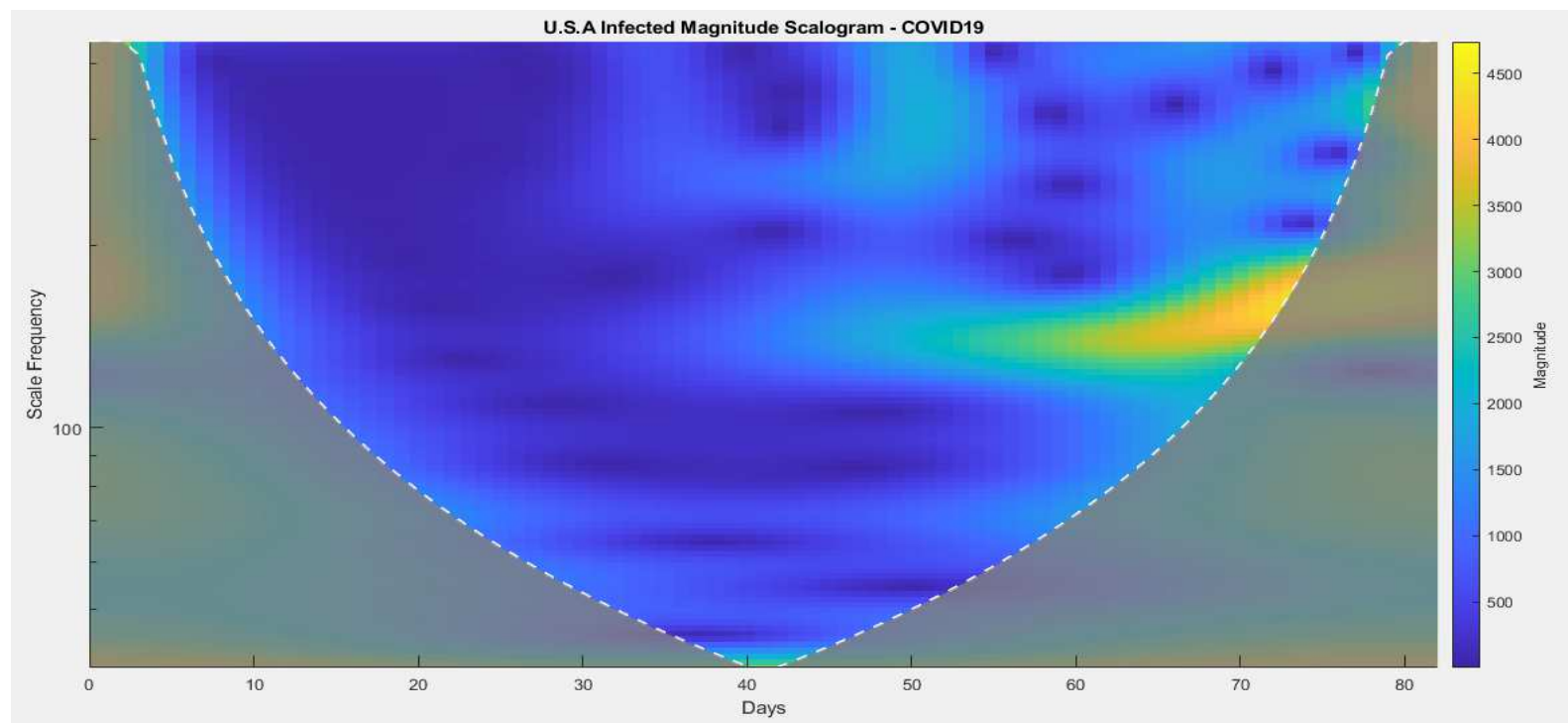

Figure 6: COVID19 - U.S.A Daily Infected Cases Wavelet Magnitude Scalogram, the date is from $2 / 15 / 2020$ to $5 / 7 / 2020$ 


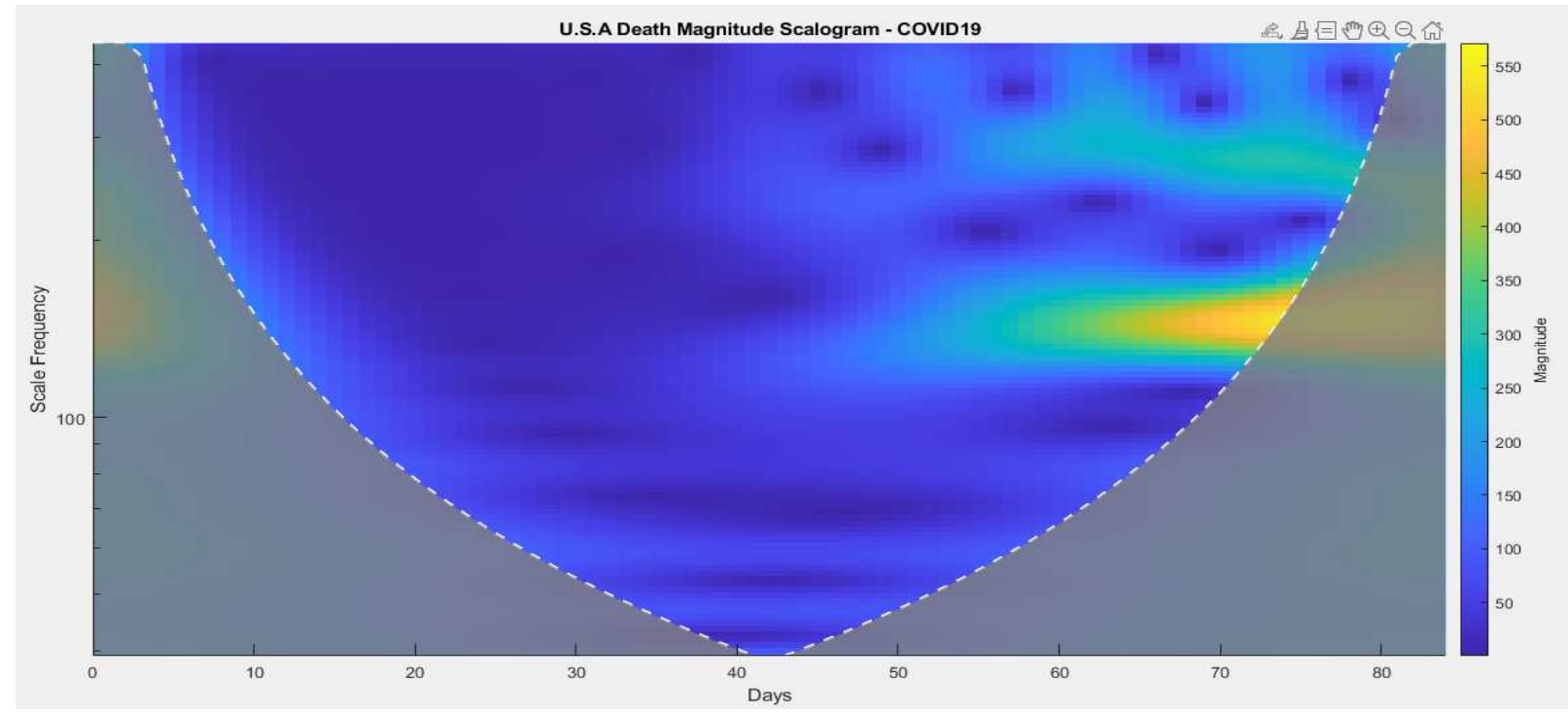

Figure 7: COVID19 - USA Daily Deaths Wavelet Magnitude Scalogram, the date is from $2 / 15 / 2020$ to $5 / 7 / 2020$.

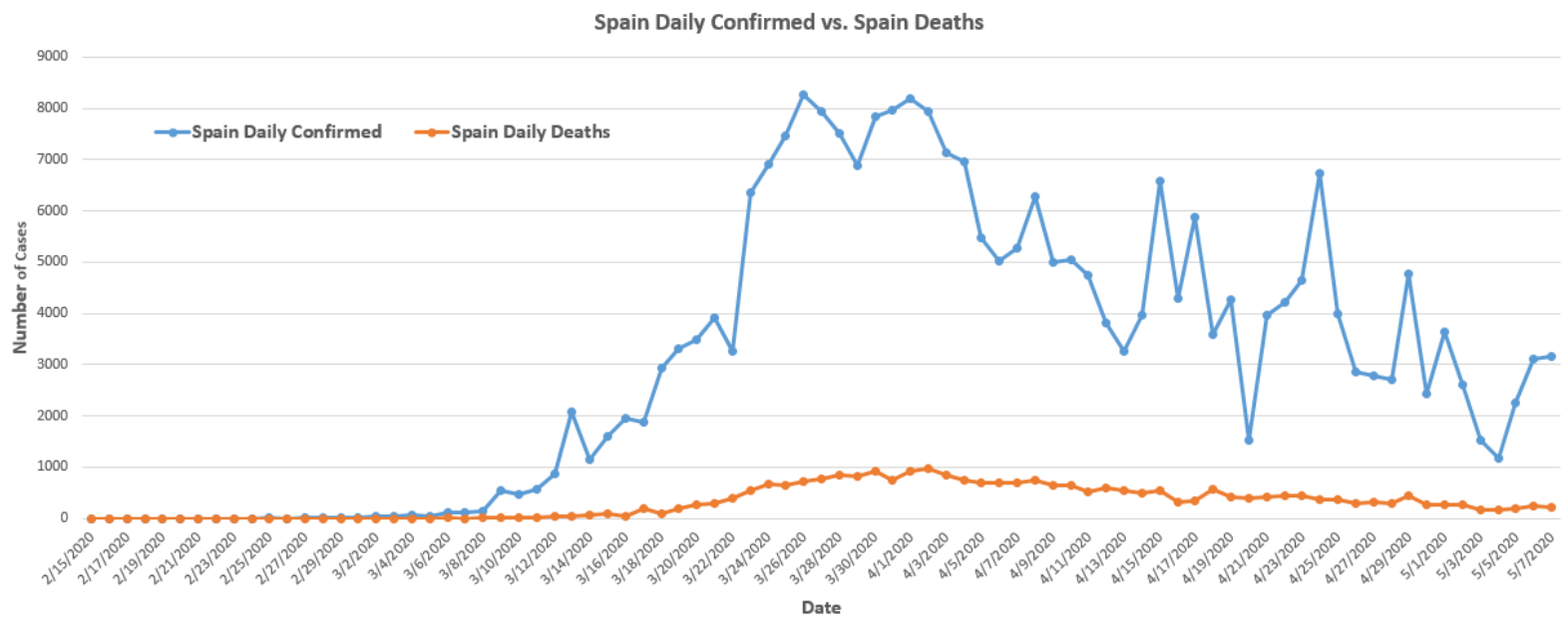

Figure 8: COVID19 - Spain Daily Infected Cases and Deaths, the date is from 2/15/2020 to $5 / 7 / 2020$. 


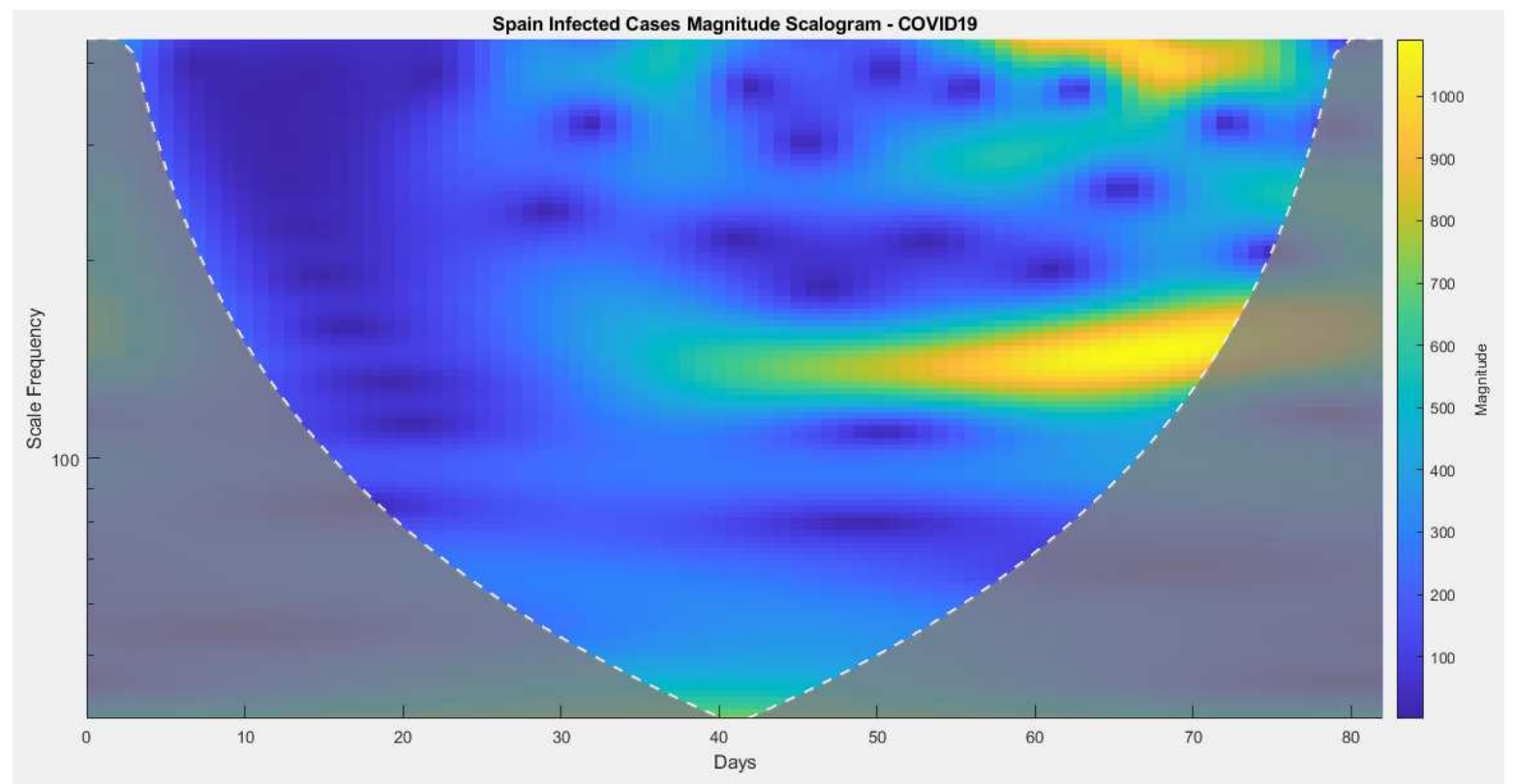

Figure 9: COVID19 - Spain Daily Infected Cases Wavelet Magnitude Scalogram, the date is from $2 / 15 / 2020$ to $5 / 7 / 2020$

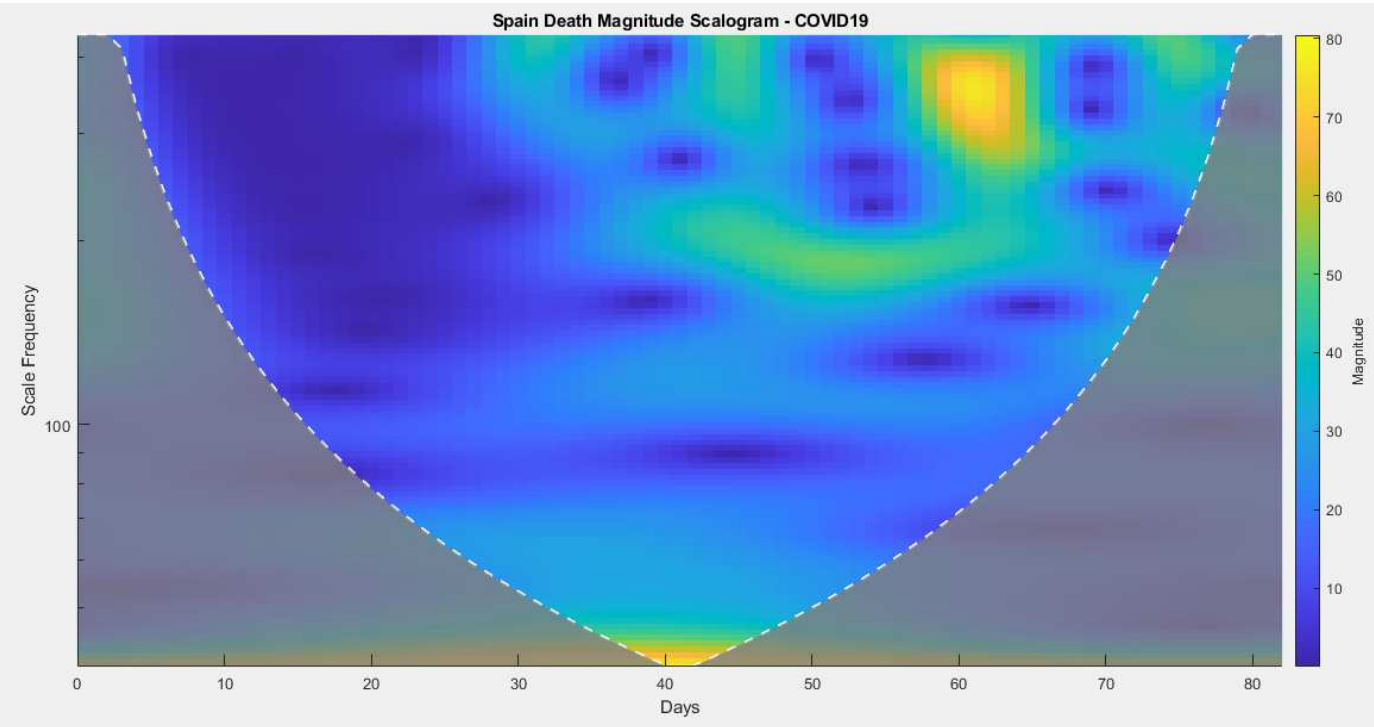

Figure 10: COVID19 - Spain Daily Deaths Wavelet Magnitude Scalogram, the date is from $2 / 15 / 2020$ to $5 / 7 / 2020$. 


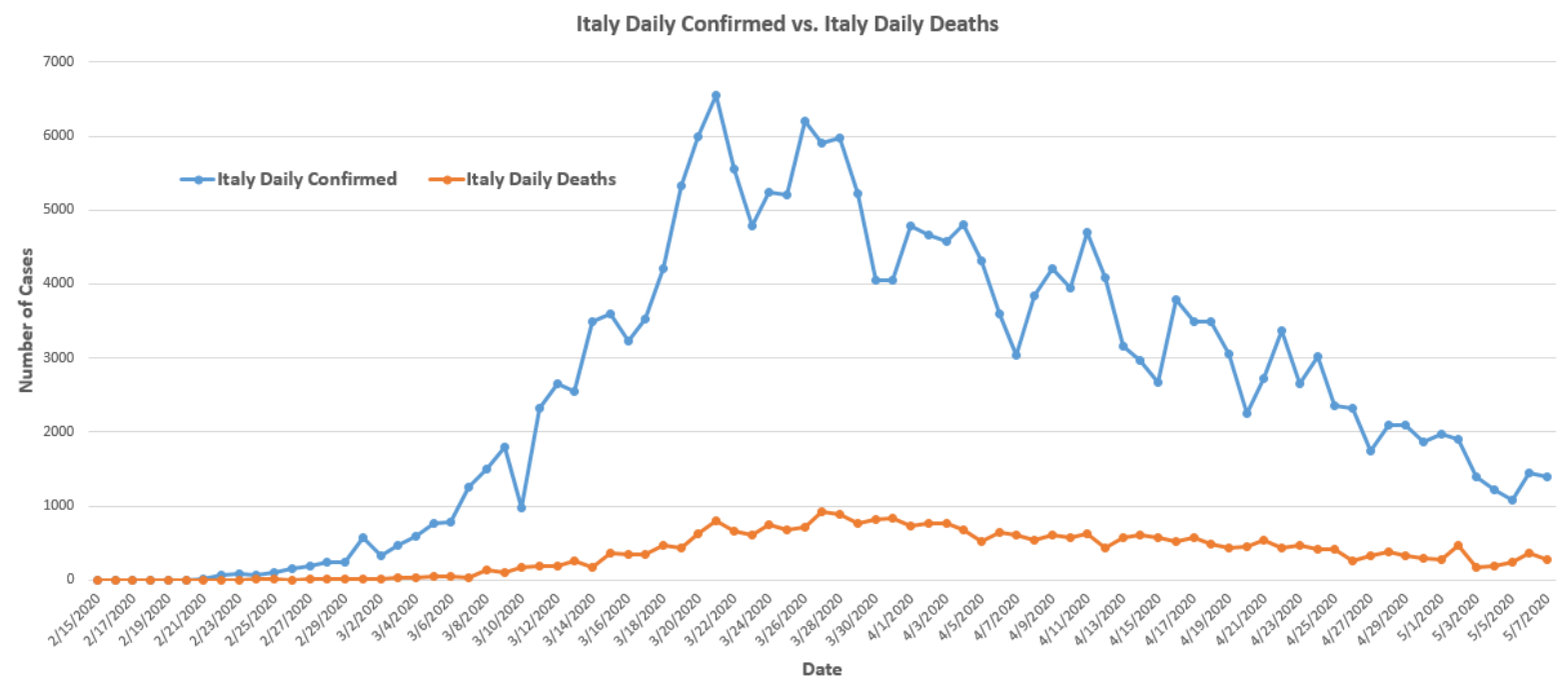

Figure 11: COVID19 - Italy Daily Infected Cases and Deaths, the date is from 2/15/2020 to 5/7/2020.

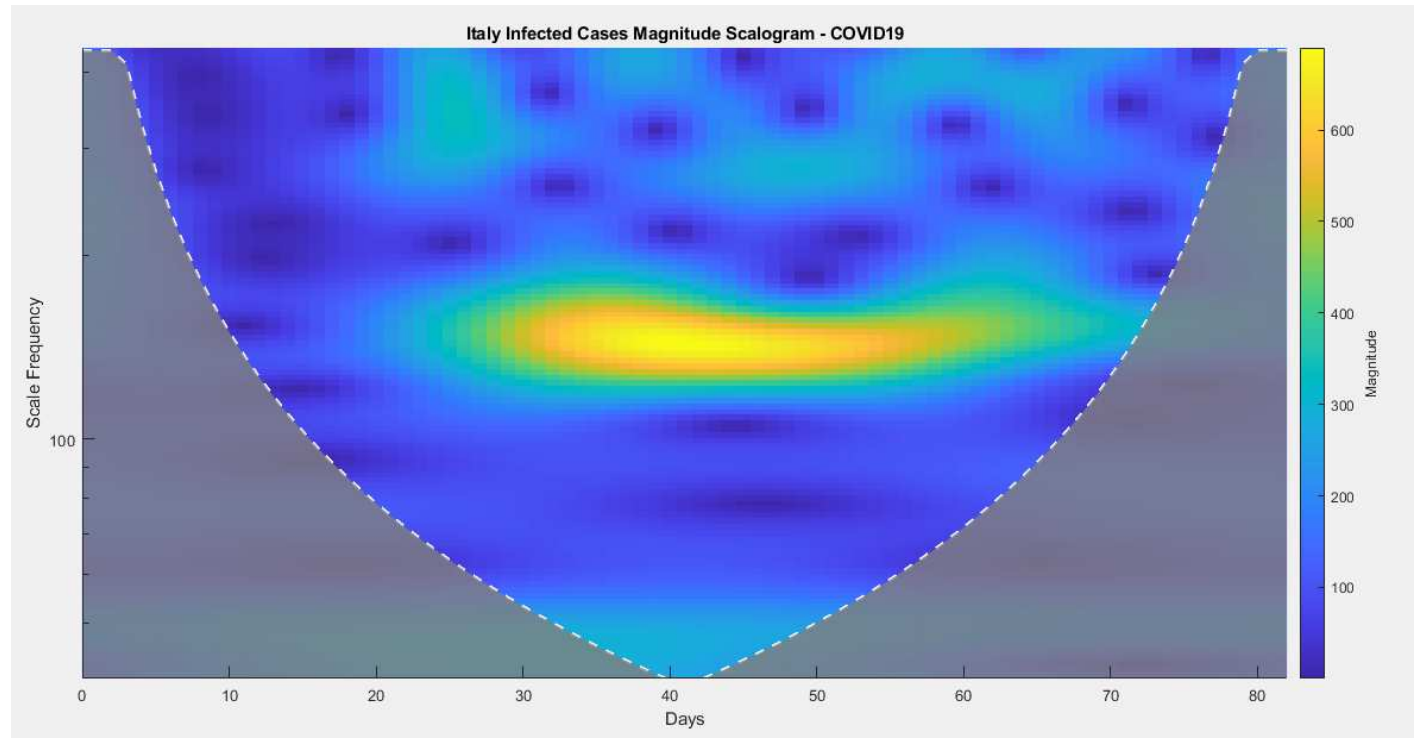

Figure 12: COVID19 - Italy Daily Infected Cases Wavelet Magnitude Scalogram, the date is from $2 / 15 / 2020$ to $5 / 7 / 2020$ 


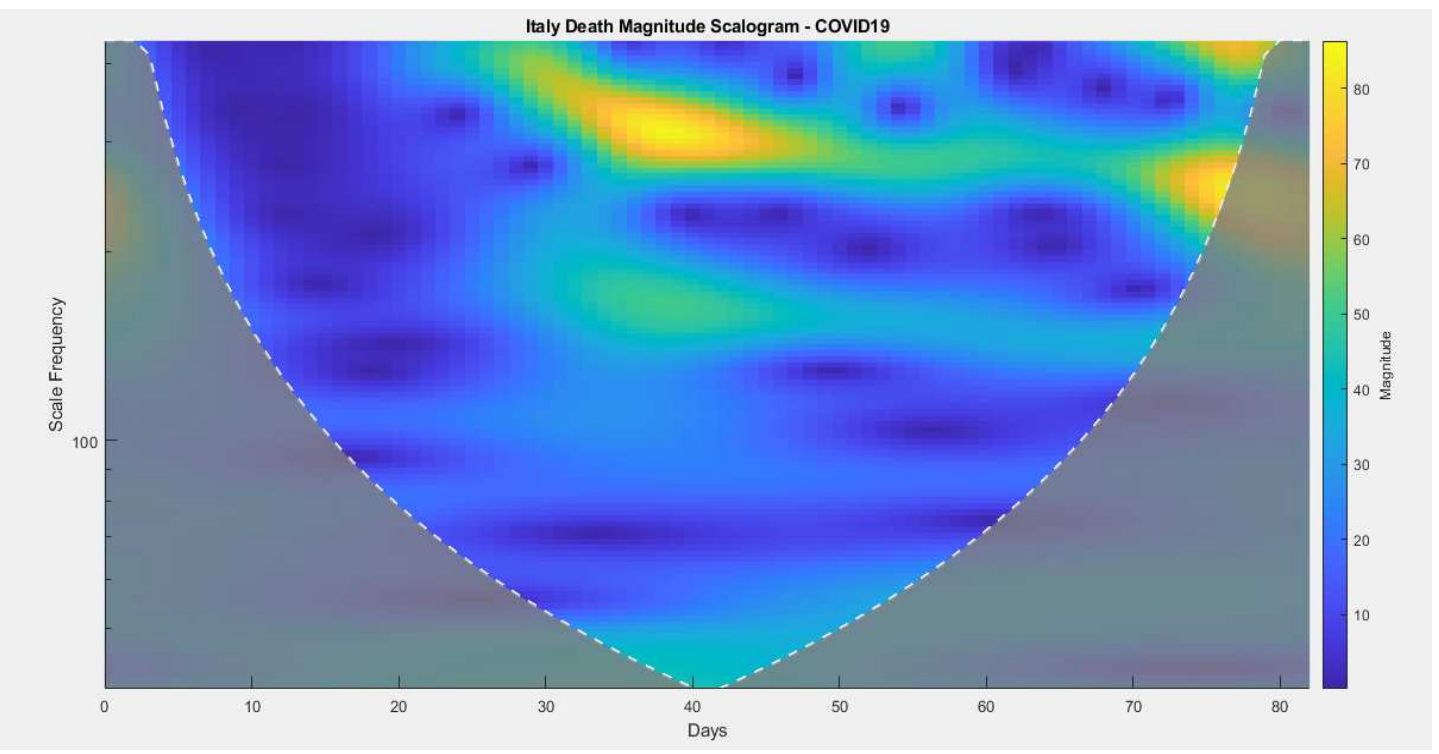

Figure 13: COVID19 - Italy Daily Deaths Wavelet Magnitude Scalogram, the date is from $2 / 15 / 2020$ to $5 / 7 / 2020$.

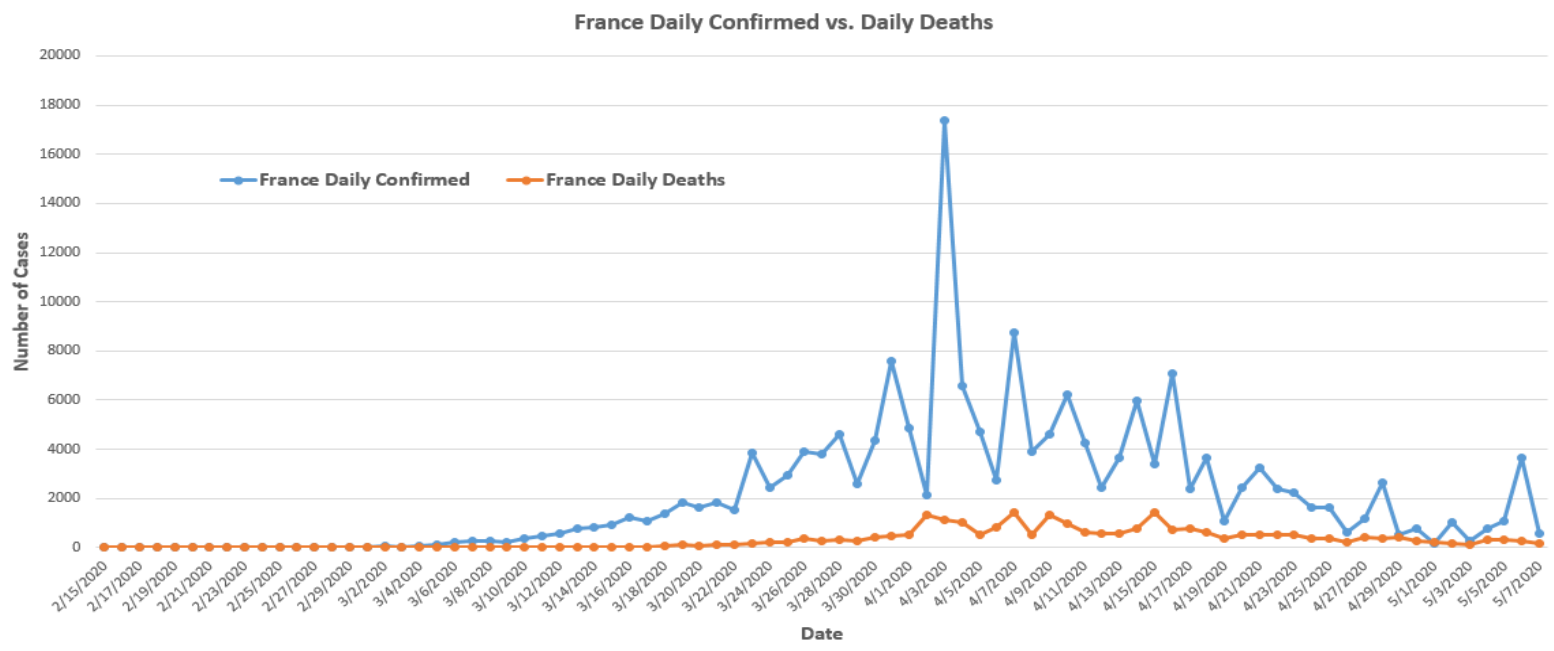

Figure 14: COVID19 - France Daily Infected Cases and Deaths, the date is from 2/15/2020 to $5 / 7 / 2020$. 


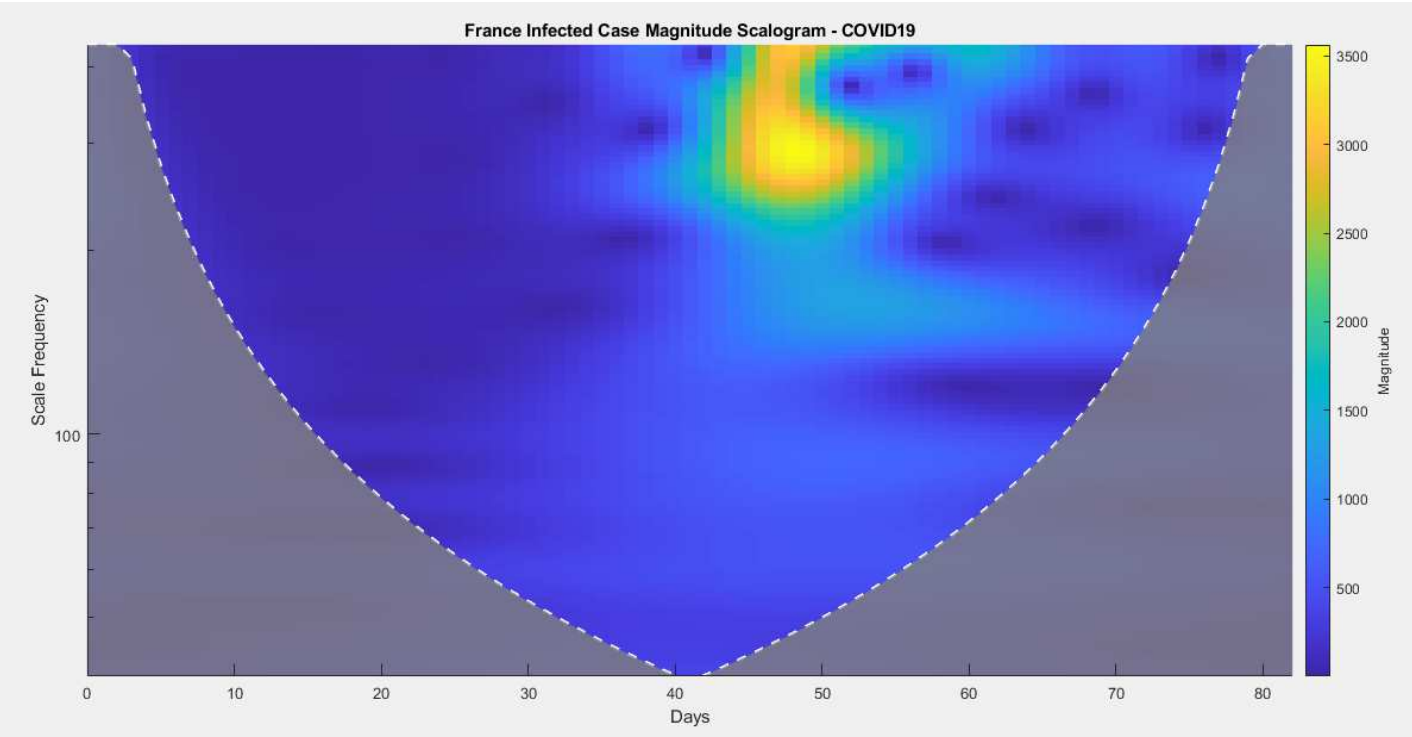

Figure 15: COVID19 - France Daily Infected Cases Wavelet Magnitude Scalogram, the date is from $2 / 15 / 2020$ to $5 / 7 / 2020$

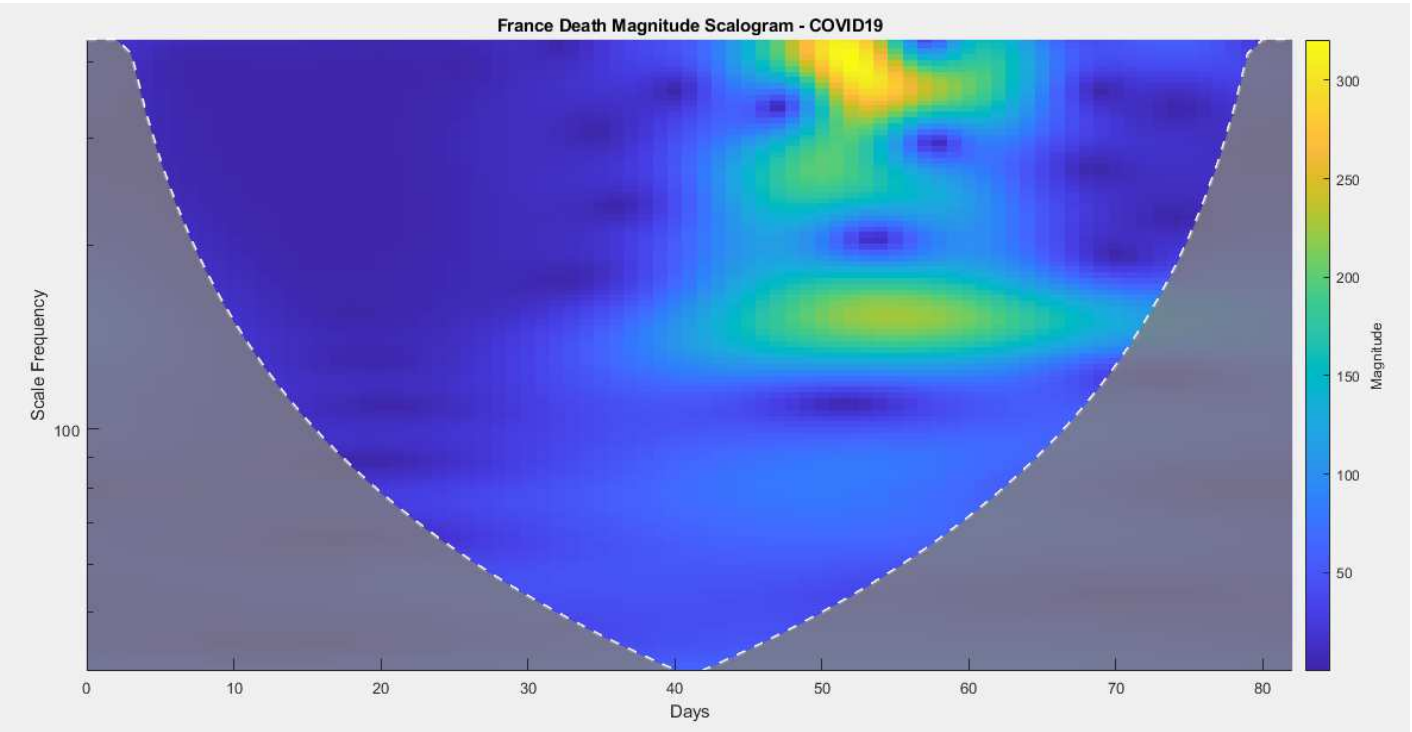

Figure 16: COVID19 - France Daily Deaths Wavelet Magnitude Scalogram, the date is from $2 / 15 / 2020$ to $5 / 7 / 2020$. 


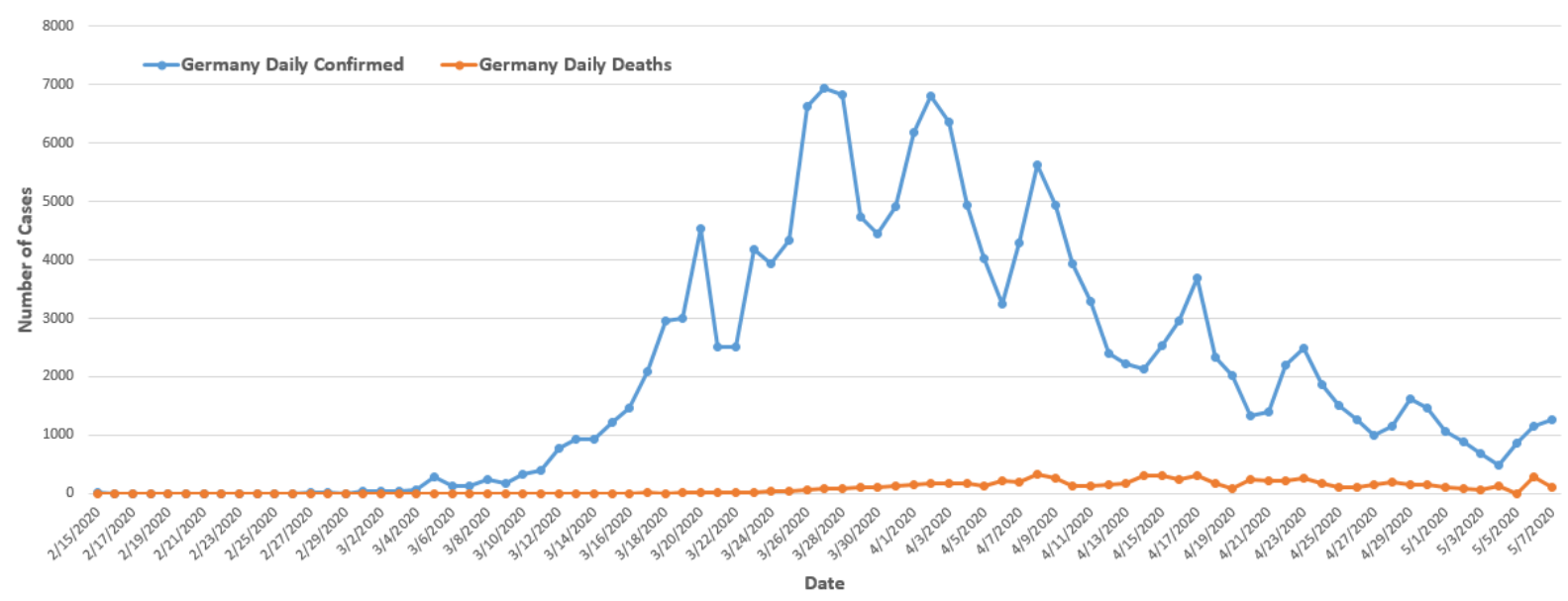

Figure 17: COVID19 - Germany Daily Infected Cases and Deaths, the date is from $2 / 15 / 2020$ to $5 / 7 / 2020$.

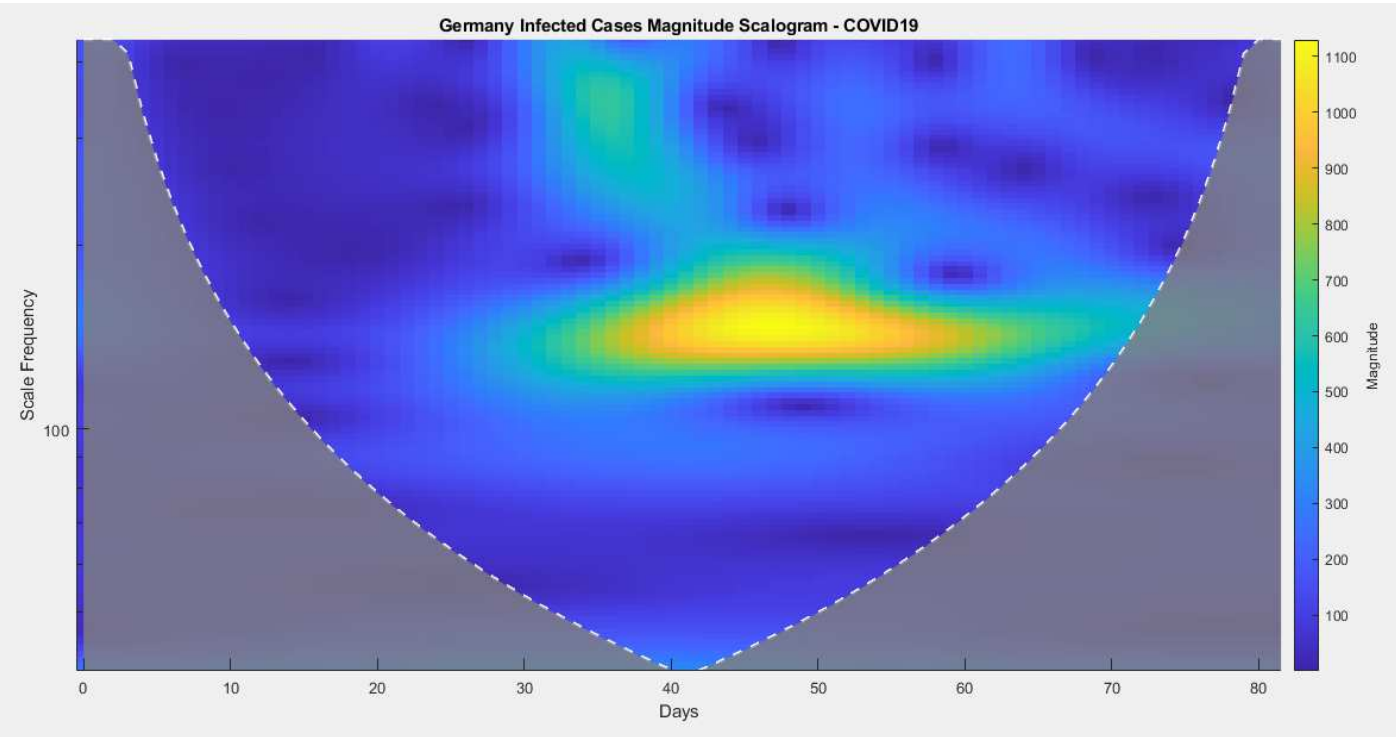

Figure 18: COVID19 - Germany Daily Infected Cases Wavelet Magnitude Scalogram, the date is from $2 / 15 / 2020$ to $5 / 7 / 2020$ 


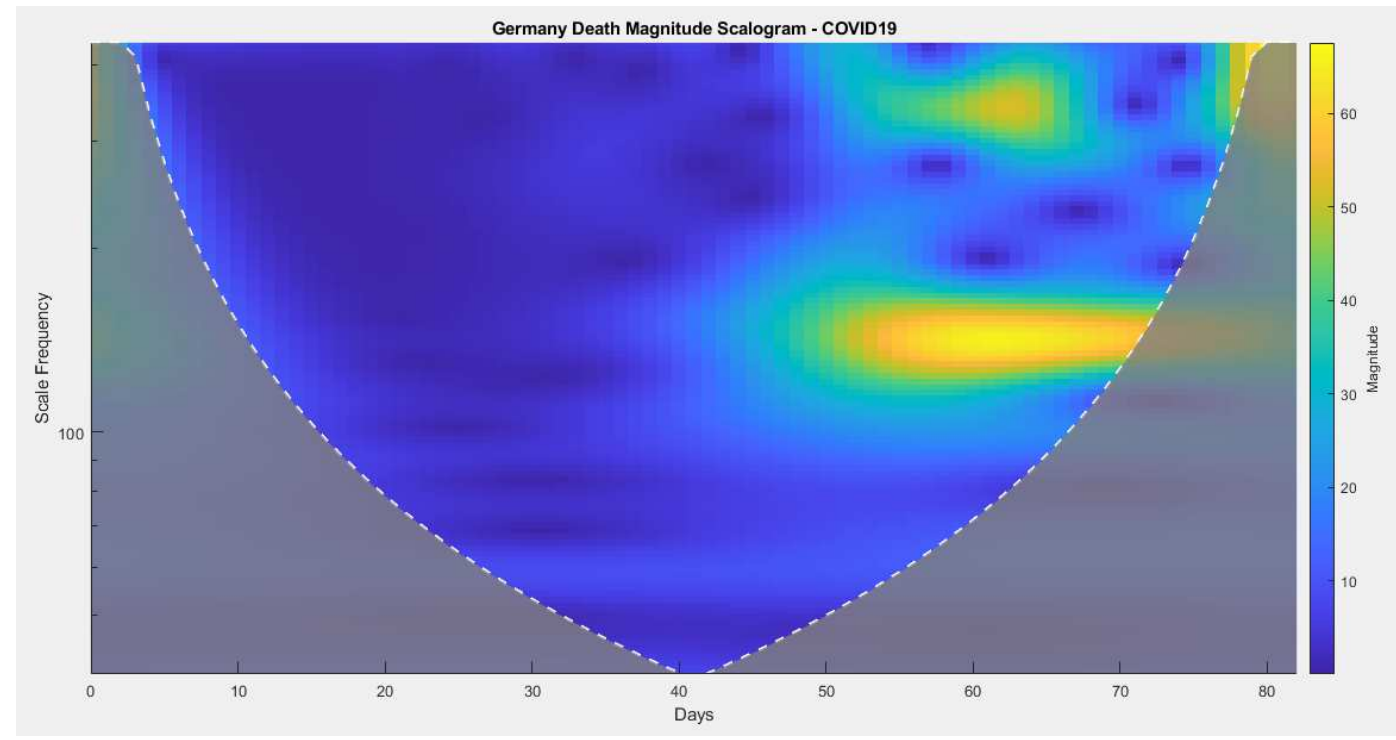

Figure 19: COVID19 - Germany Daily Deaths Wavelet Magnitude Scalogram, the date is from $2 / 15 / 2020$ to $5 / 7 / 2020$.

U.K. Daily Confirmed vs. Daily Deaths

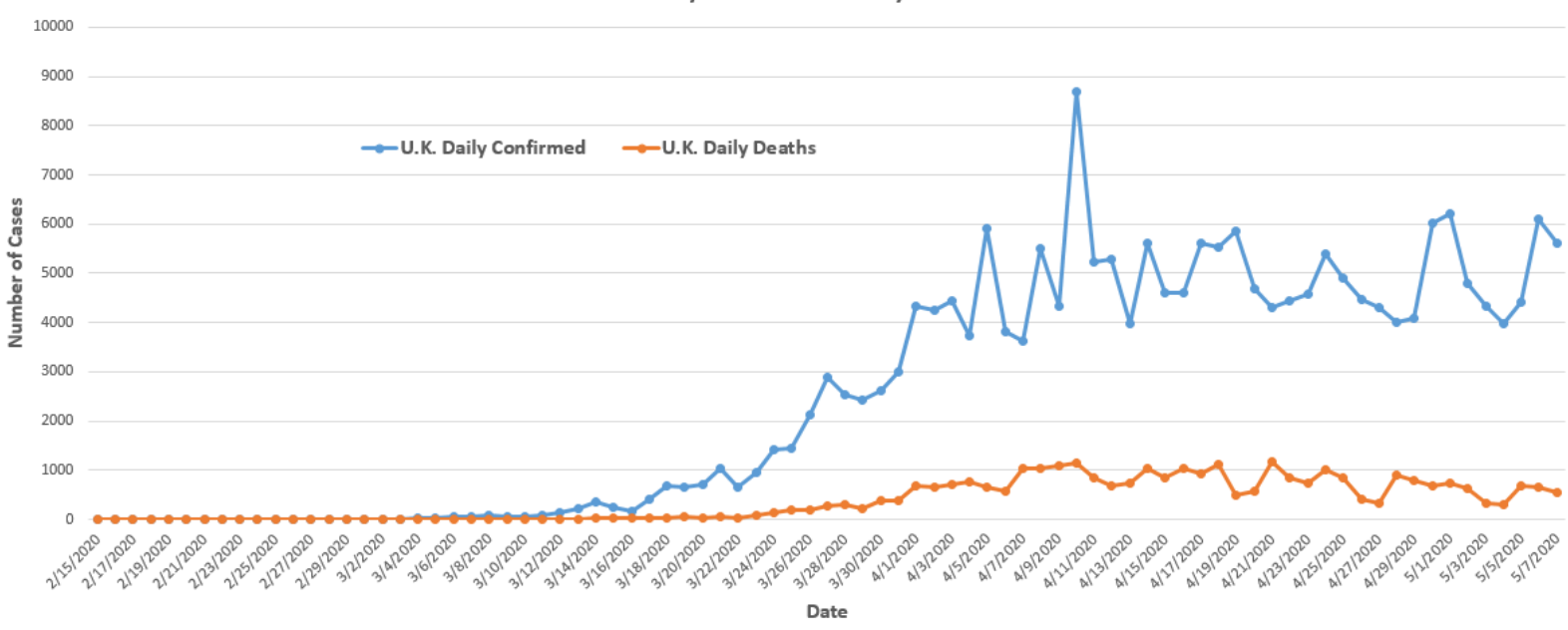

Figure 20: COVID19 - U.K. Daily Infected Cases and Deaths, the date is from 2/15/2020 to $5 / 7 / 2020$. 


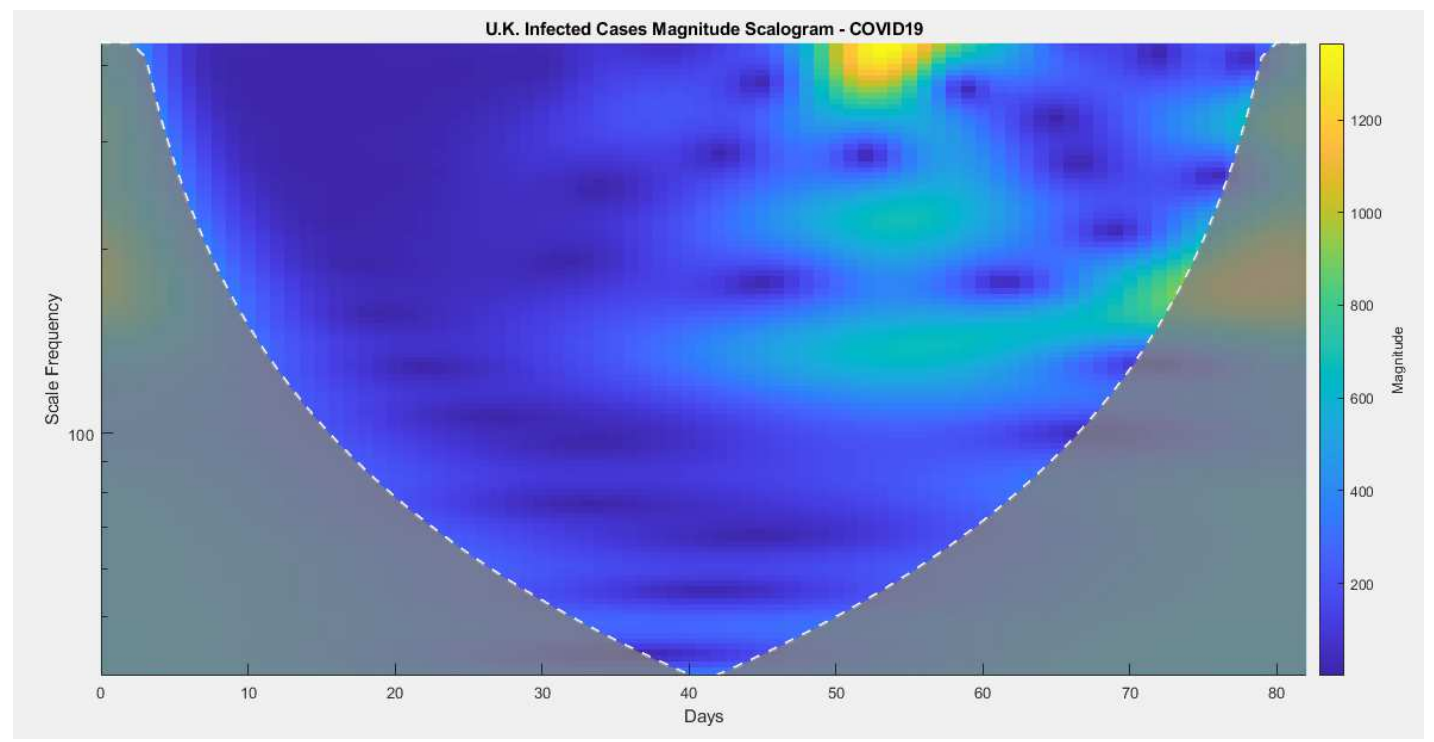

Figure 21: COVID19 - U.K. Daily Infected Cases Wavelet Magnitude Scalogram, the date is from $2 / 15 / 2020$ to $5 / 7 / 2020$

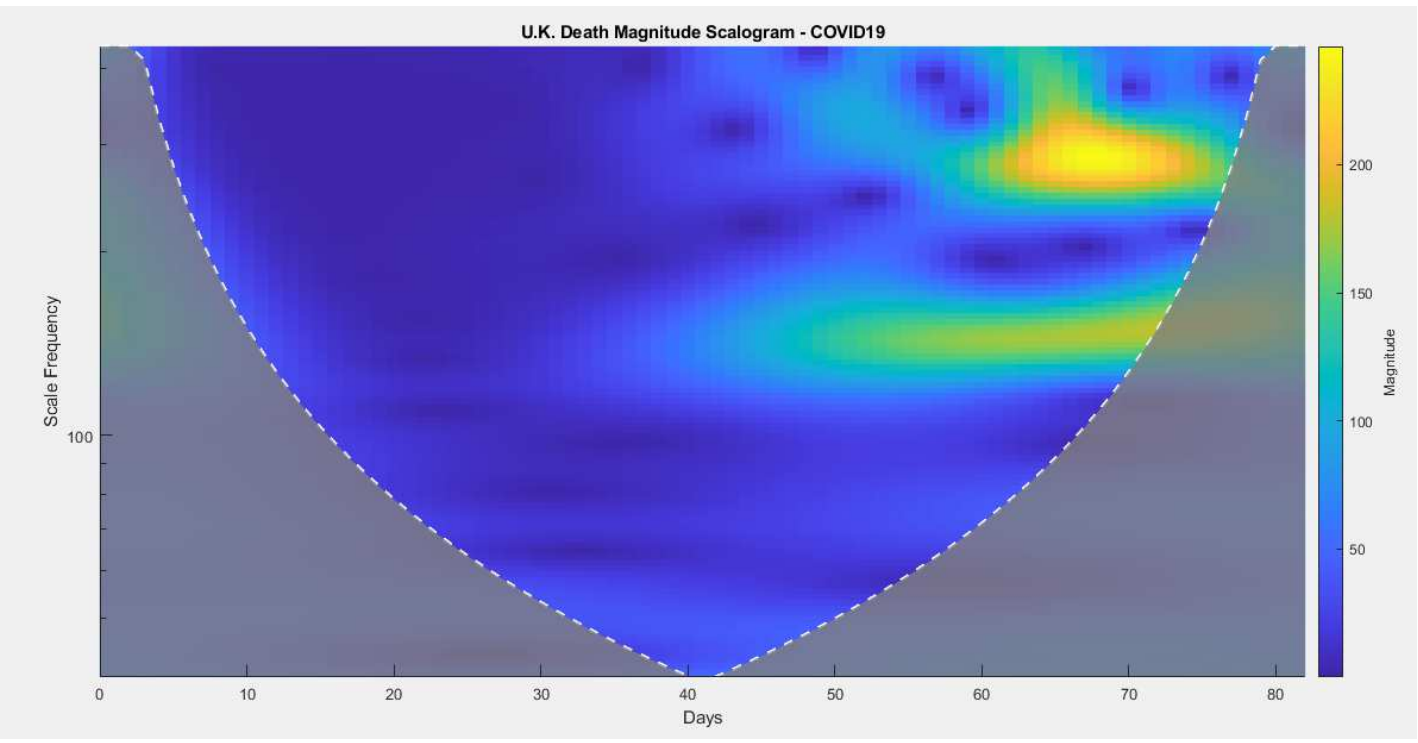

Figure 22: COVID19 - U.K. Daily Deaths Wavelet Magnitude Scalogram, the date is from $2 / 15 / 2020$ to $5 / 7 / 2020$. 
S. Korea Daily Confirmed vs. Daily Deaths

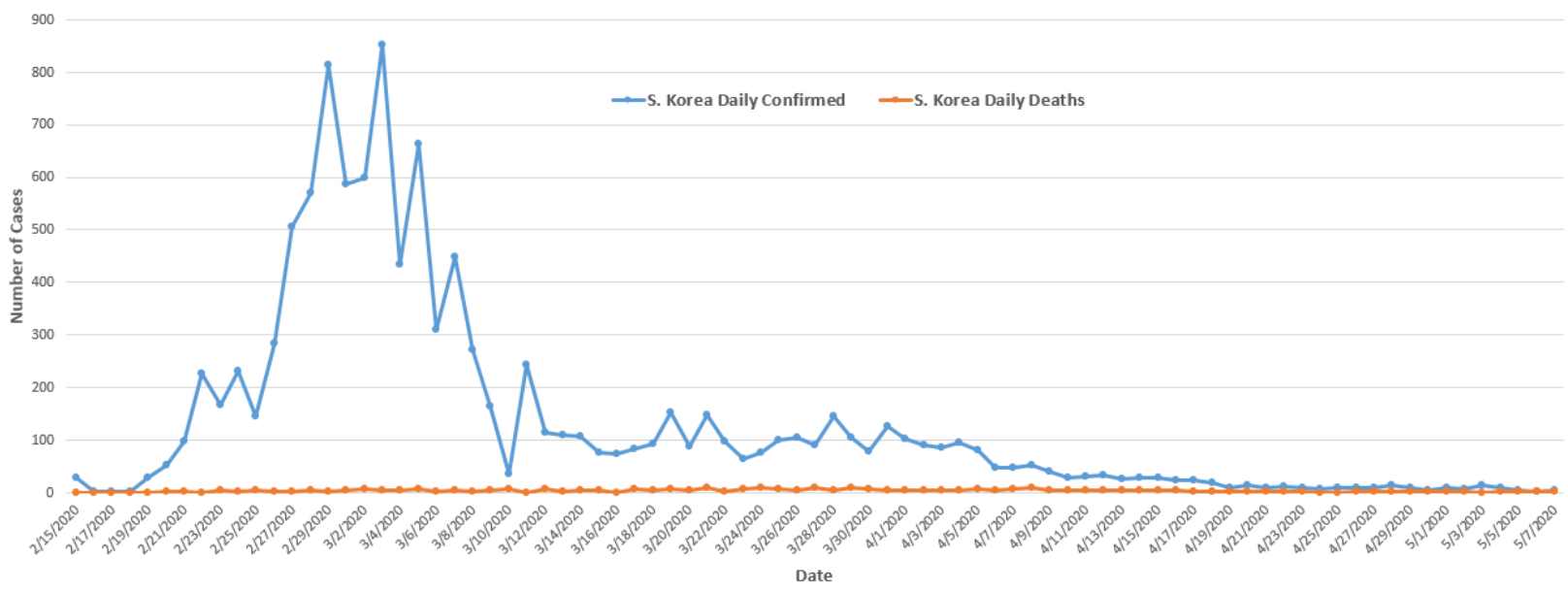

Figure 23: COVID19 - South Korea Daily Infected Cases and Deaths, the date is from 2/15/2020 to 5/7/2020.

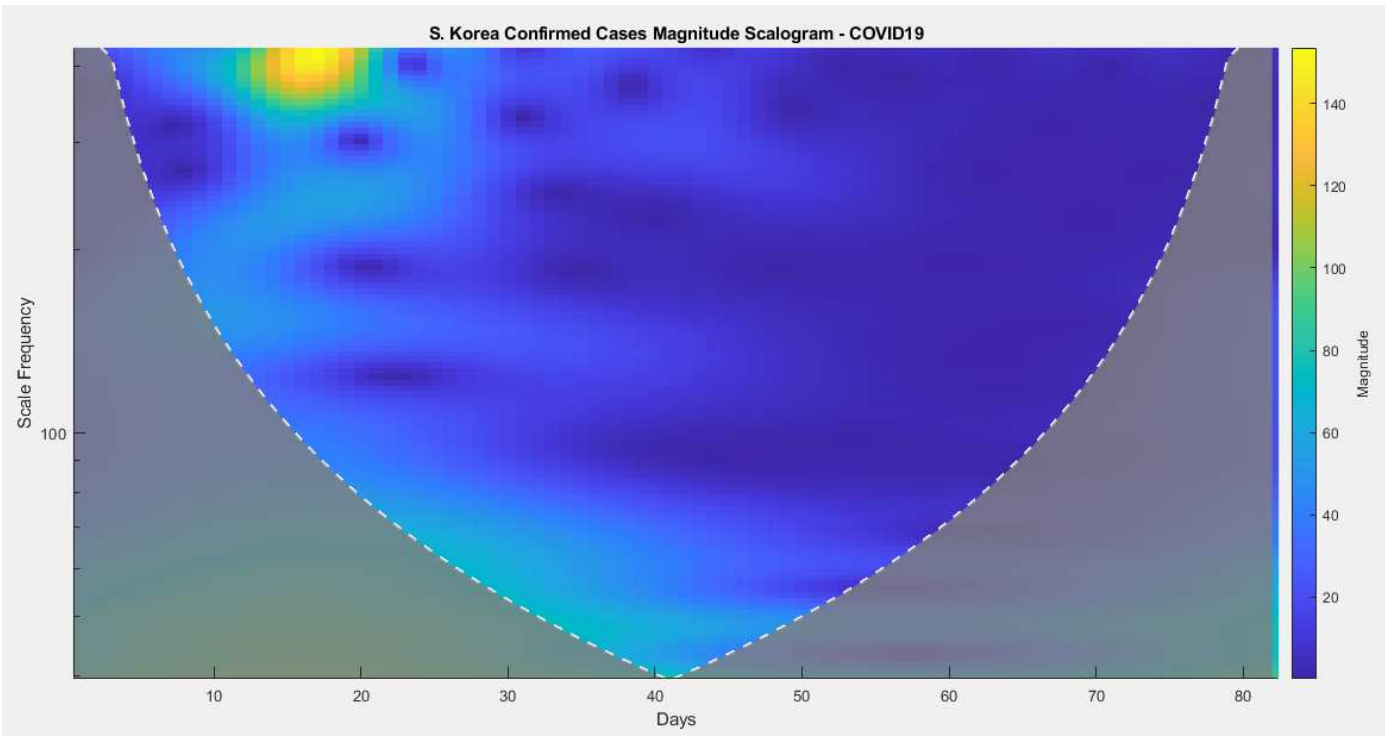

Figure 24: COVID19 - South Korea Daily Infected Cases Wavelet Magnitude Scalogram, the date is from $2 / 15 / 2020$ to $5 / 7 / 2020$ 


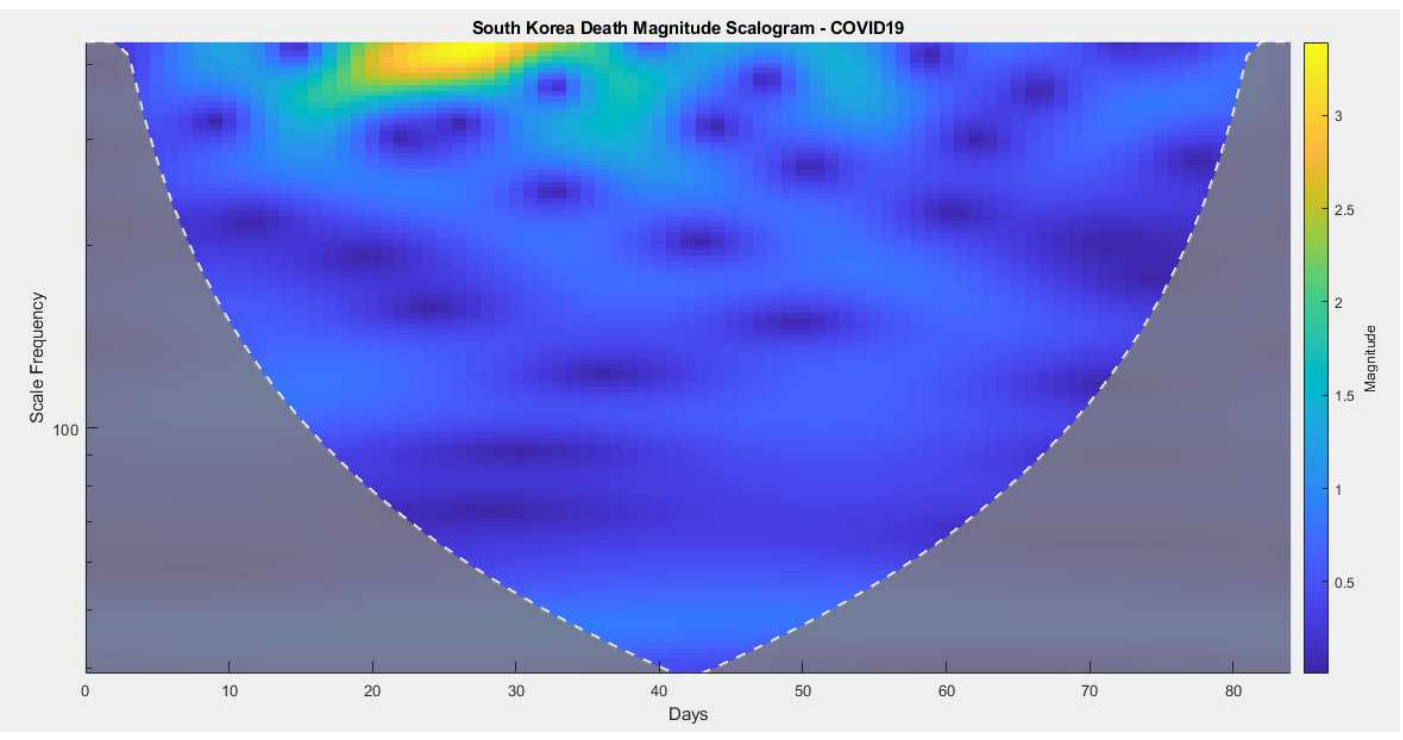

Figure 25: COVID19 - South Korea Daily Deaths Wavelet Magnitude Scalogram, the date is from $2 / 15 / 2020$ to $5 / 7 / 2020$.

Michigan Daily Confirmed vs Daily Deaths

2500

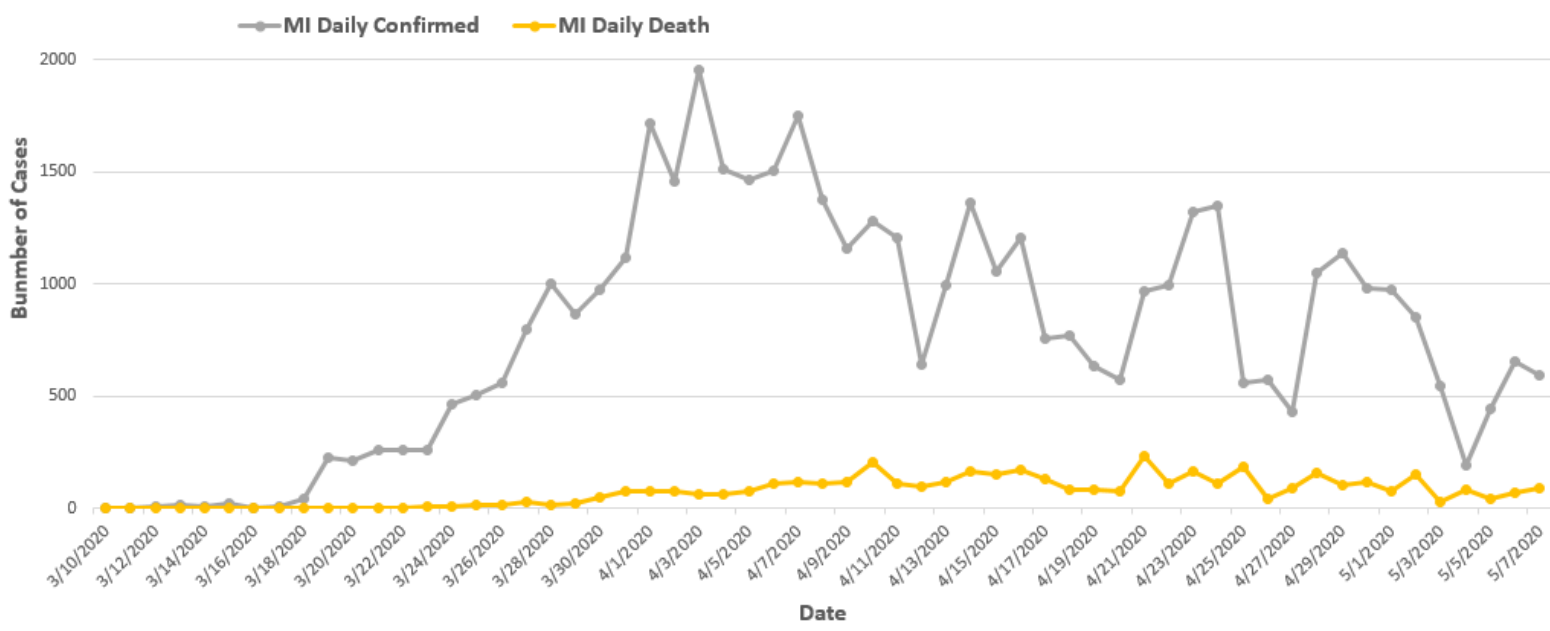

5 Figure 26: COVID19 - Michigan Daily Infected Cases and Deaths, the date is from $3 / 10 / 2020$ to $5 / 7 / 2020$. 


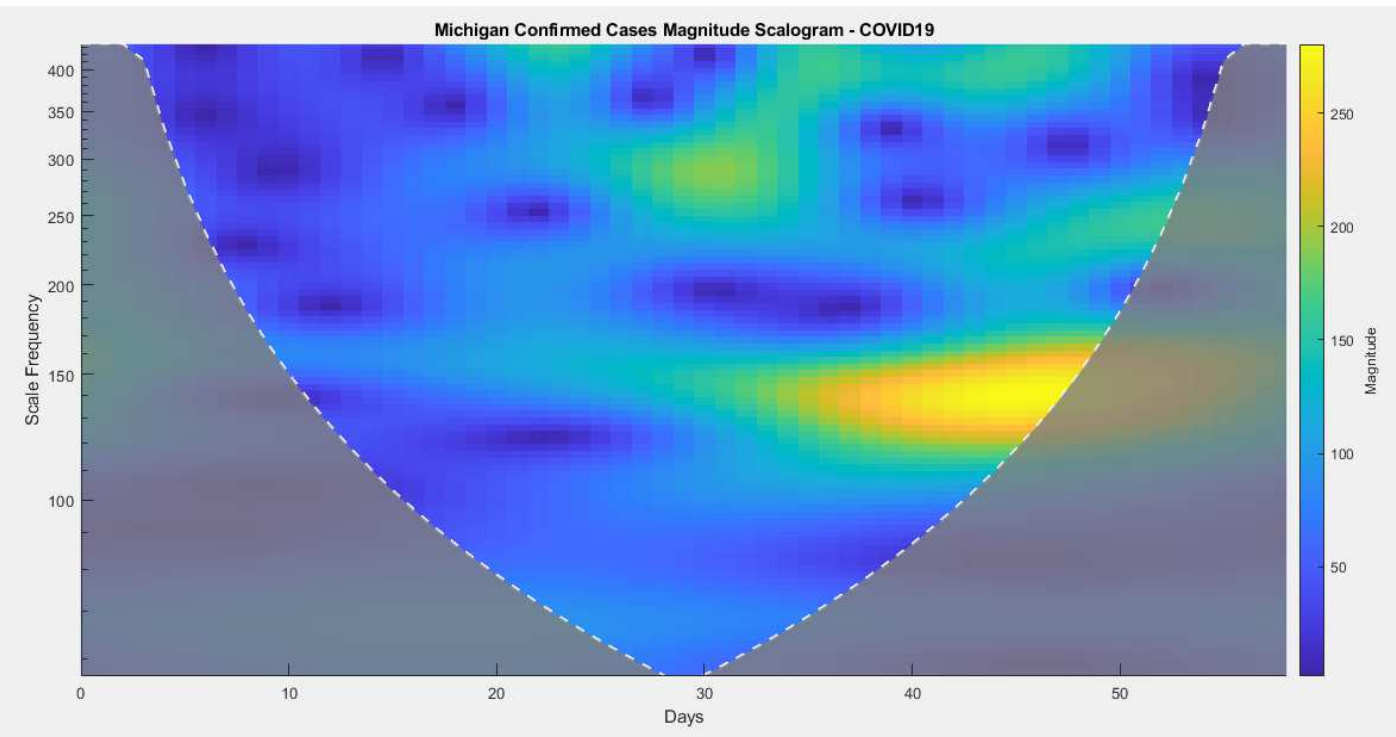

Figure 27: COVID19 - Michigan Daily Infected Cases Wavelet Magnitude Scalogram, the date is from $3 / 10 / 2020$ to $5 / 7 / 2020$

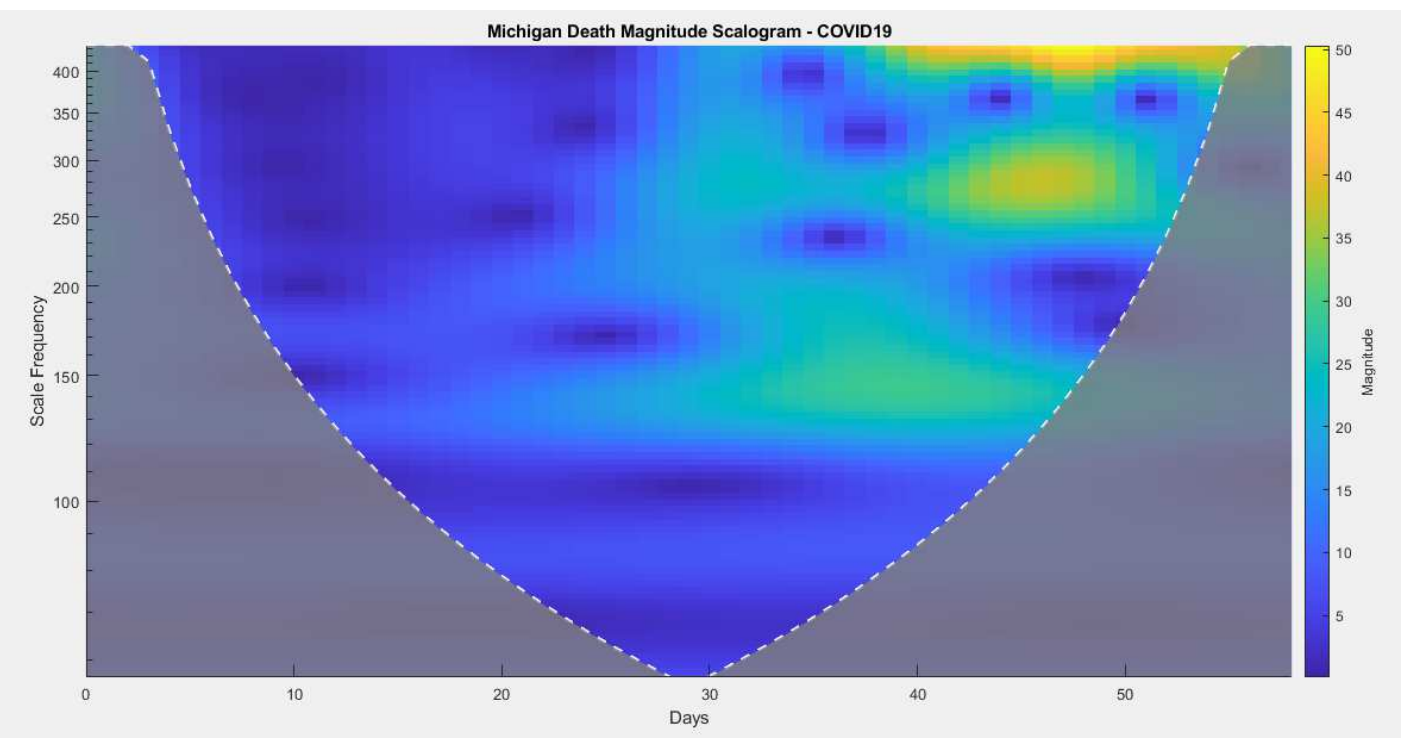

$5 \quad$ Figure 28: COVID19 - Michigan Daily Deaths Wavelet Magnitude Scalogram, the date is from $3 / 10 / 2020$ to $5 / 7 / 2020$. 


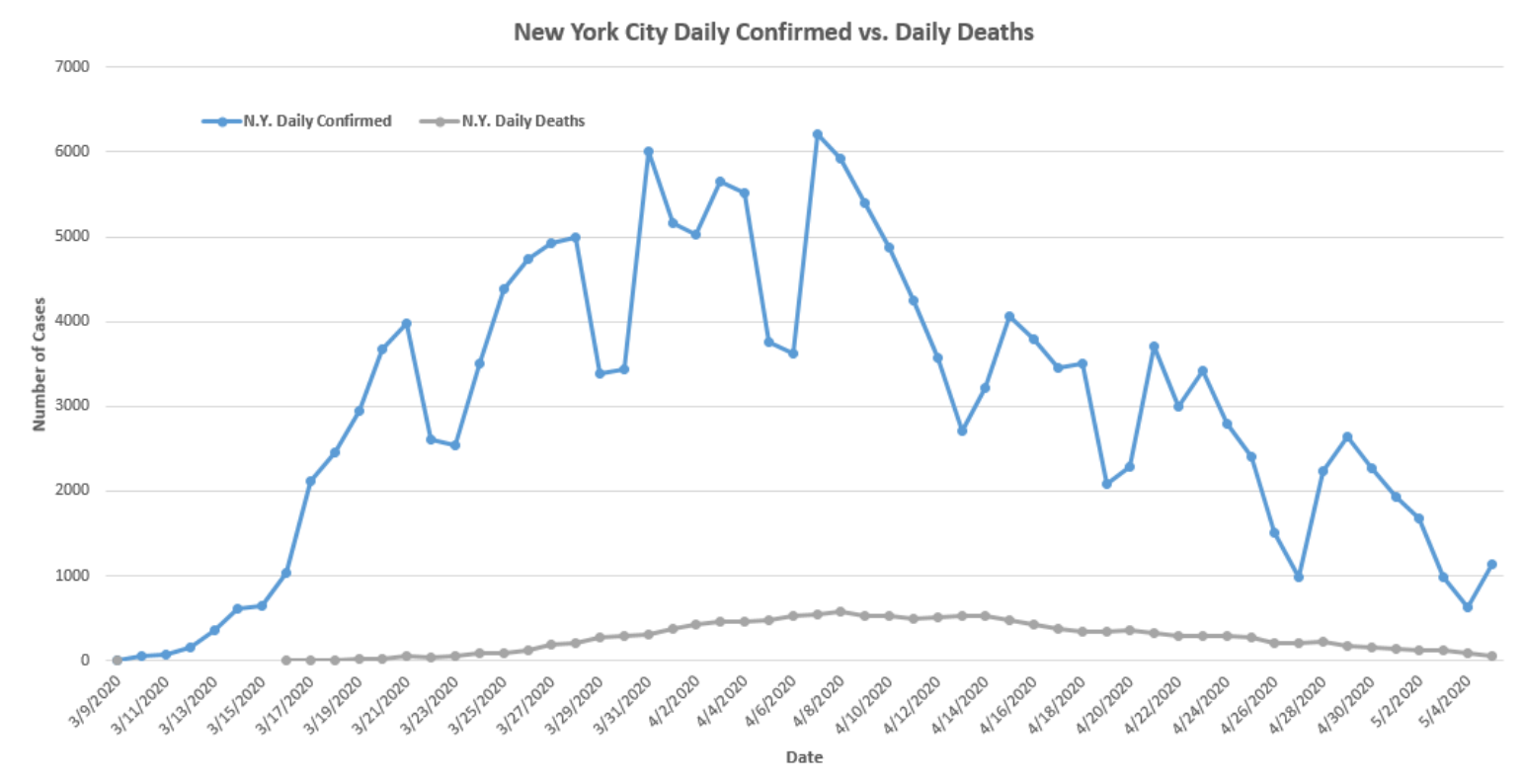

Figure 29: COVID19 - New York City Daily Infected Cases and Deaths, the date is from $3 / 9 / 2020$ to $5 / 5 / 2020$.

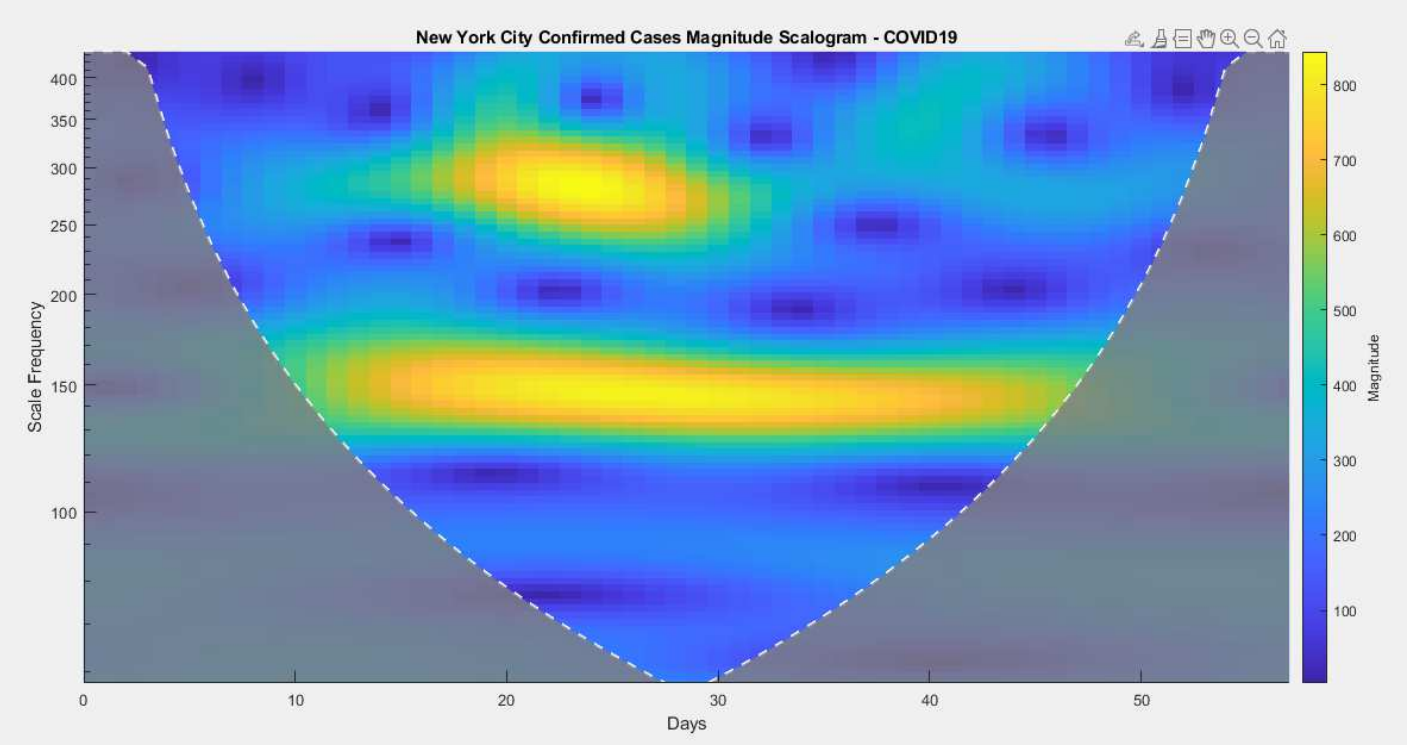

Figure 30: COVID19 - New York City Daily Infected Cases Wavelet Magnitude Scalogram, the date is from $3 / 9 / 2020$ to $5 / 5 / 2020$ 


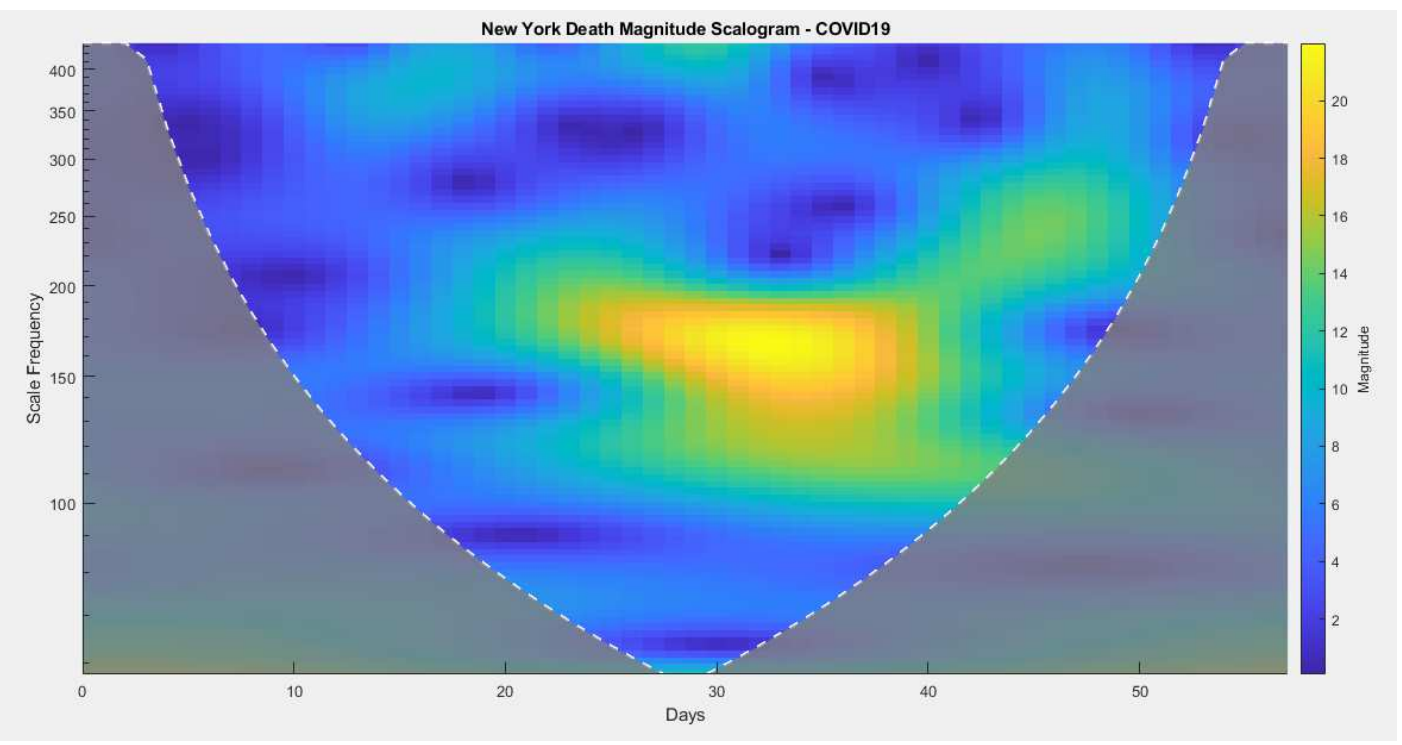

Figure 31: COVID19 - New York City Daily Deaths Wavelet Magnitude Scalogram, the date is from $3 / 9 / 2020$ to $5 / 5 / 2020$.

\begin{tabular}{|c|c|c|c|c|c|c|c|c|c|c|c|c|}
\hline \multirow{3}{*}{ Countries/Region } & \multicolumn{6}{|c|}{ Spreading Pattern } & \multicolumn{6}{|c|}{ Death Pattern } \\
\hline & \multicolumn{3}{|c|}{ Highest Magnitude } & \multicolumn{3}{|c|}{ Medium Magnitude } & \multicolumn{3}{|c|}{ Highest Magnitude } & \multicolumn{3}{|c|}{ Medium Magnitude } \\
\hline & Magnitude & Scale & \begin{tabular}{|l} 
Time Period \\
\end{tabular} & Magnitude & Scale & Time Period & Magnitude & Scale & Time Period & Magnitude & Scale & \begin{tabular}{|l|} 
Time Period \\
\end{tabular} \\
\hline China & 2000 to 2500 & 329 to 434 & $2 / 12 / 2020$ & 1500 to 2000 & 189 to 329 & $2 / 12 / 2020$ & 320 & 329 to 434 & $4 / 17 / 2020$ & 200 to 250 & 206 to 329 & $4 / 13$ to $4 / 19$ \\
\hline \multirow[b]{2}{*}{ U.S.A } & \multirow[b]{2}{*}{4000 to 4500} & \multirow[b]{2}{*}{137 to 183} & \multirow[b]{2}{*}{$4 / 25$ to $4 / 28$} & 3000 & 329 & $5 / 2 / 2020$ & \multirow[b]{2}{*}{500 to 550} & \multirow[b]{2}{*}{137 to 164} & \multirow[b]{2}{*}{$4 / 27$ to $4 / 29$} & \multirow[b]{2}{*}{350 to 400} & 274 & after $5 / 2$ \\
\hline & & & & 1500 to 2500 & 149 & after $3 / 25$ & & & & & 150 & after $4 / 4$ \\
\hline \multirow[b]{2}{*}{ Spain } & \multirow[b]{2}{*}{900 to 1070} & 137 to 164 & $4 / 7$ to $4 / 27$ & \multirow[b]{2}{*}{600 to 700} & \multirow[b]{2}{*}{ Varies Scale } & \multirow[b]{2}{*}{$3 / 20$ to $5 / 1 / 2020$} & \multirow[b]{2}{*}{70 to 80} & 274 to 411 & $4 / 11$ to $4 / 19$ & \multirow[b]{2}{*}{50} & \multirow[b]{2}{*}{ Varies scale } & \multirow[b]{2}{*}{ after $3 / 15$} \\
\hline & & 412 & $4 / 14$ to $4 / 24$ & & & & & 42 & $3 / 25 / 2020$ & & & \\
\hline \multirow{2}{*}{ Italy } & \multirow{2}{*}{600 to 700} & \multirow{2}{*}{126 to 164} & \multirow{2}{*}{$3 / 17$ to $4 / 9$} & \multirow{2}{*}{400} & \multirow{2}{*}{ Varies Scale } & \multirow{2}{*}{$3 / 12$ to $4 / 26$} & \multirow{2}{*}{75 to 85} & 274 to 329 & $3 / 20$ to $3 / 30$ & \multirow{2}{*}{60 to 70} & \multirow{2}{*}{ Varies scale } & after $3 / 15$ \\
\hline & & & & & & & & 235 to 274 & $4 / 26$ to $5 / 1$ & & & arter $3 / 15$ \\
\hline France & 3000 to 3500 & 235 to 412 & $4 / 1$ to $4 / 7$ & 2000 to 2500 & Varies Scale & $3 / 27$ to $4 / 10$ & 280 to 320 & \begin{tabular}{|l|}
329 to 412 \\
\end{tabular} & $4 / 3$ to $4 / 9$ & 200 to 250 & Varies scale & after $3 / 30$ \\
\hline Germany & 1000 to 1100 & 137 to 164 & $3 / 23$ to $4 / 12$ & 750 to 900 & Varies scale & after $3 / 17$ & 60 to 65 & 137 to 149 & $4 / 7$ to $4 / 26$ & 60 & 412 & $5 / 3 / 2020$ \\
\hline Germany & 1000 to 1100 & 13/ to 104 & 3/23 to 4/12 & 150 to 900 & Varles scale & arter $3 / 17$ & outo tos & $13 /$ to 149 & $4 / 7104 / 20$ & 40 to 60 & Varies scale & after $4 / 1$ \\
\hline U.K. & 1200 to 1300 & 412 & $4 / 4$ to $4 / 8$ & 800 to 1100 & Varies Scale & after $3 / 31$ & 200 to 250 & 273 to 329 & $4 / 17$ to $4 / 28$ & 150 to 200 & Varies scale & after $4 / 4$ \\
\hline South Korea & 140 to 150 & 412 & $2 / 28$ to $3 / 3$ & & & & 3.5 & 412 & $3 / 4$ to $3 / 16$ & & & \\
\hline Michigan & 250 to 275 & 127 to 160 & $4 / 14$ to $4 / 25$ & 150 to 200 & Varies Scale & after $3 / 31$ & 45 to 50 & 412 & $4 / 20$ to $4 / 29$ & 35 to 40 & 235 to 325 & \begin{tabular}{|l|}
$4 / 20$ to $4 / 29$ \\
\end{tabular} \\
\hline thicingait & & & & & & & & & & 30 & Varies scale & after $4 / 8$ \\
\hline New York City & 700 to 800 & \begin{tabular}{|l|}
137 to 164 \\
236 to 277 \\
\end{tabular} & $3 / 20$ to $4 / 23$ & 500 to 650 & Varies Scale & $3 / 18$ to $4 / 23$ & 18 to 22 & 137 to 183 & $4 / 1$ to $4 / 17$ & 14to 18 & Varies scale & after $3 / 28$ \\
\hline
\end{tabular}

Table 1. The Summary of Wavelet Magnitude, Scale and Time for daily infected cases and deaths at China, U.S.A. Spain, Italy, France, Germany, U.K., South Korea, Michigan and New York City. 
Figures
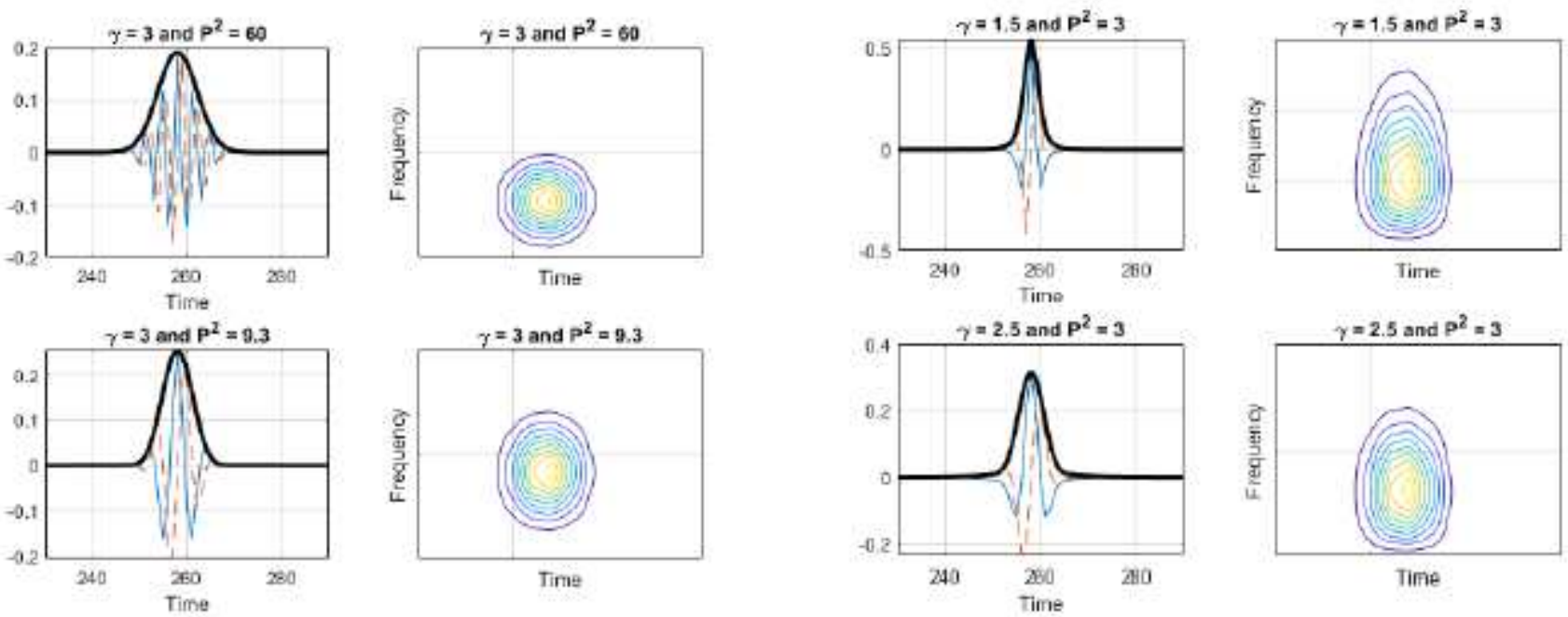

\section{Figure 1}

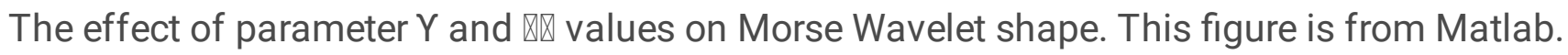

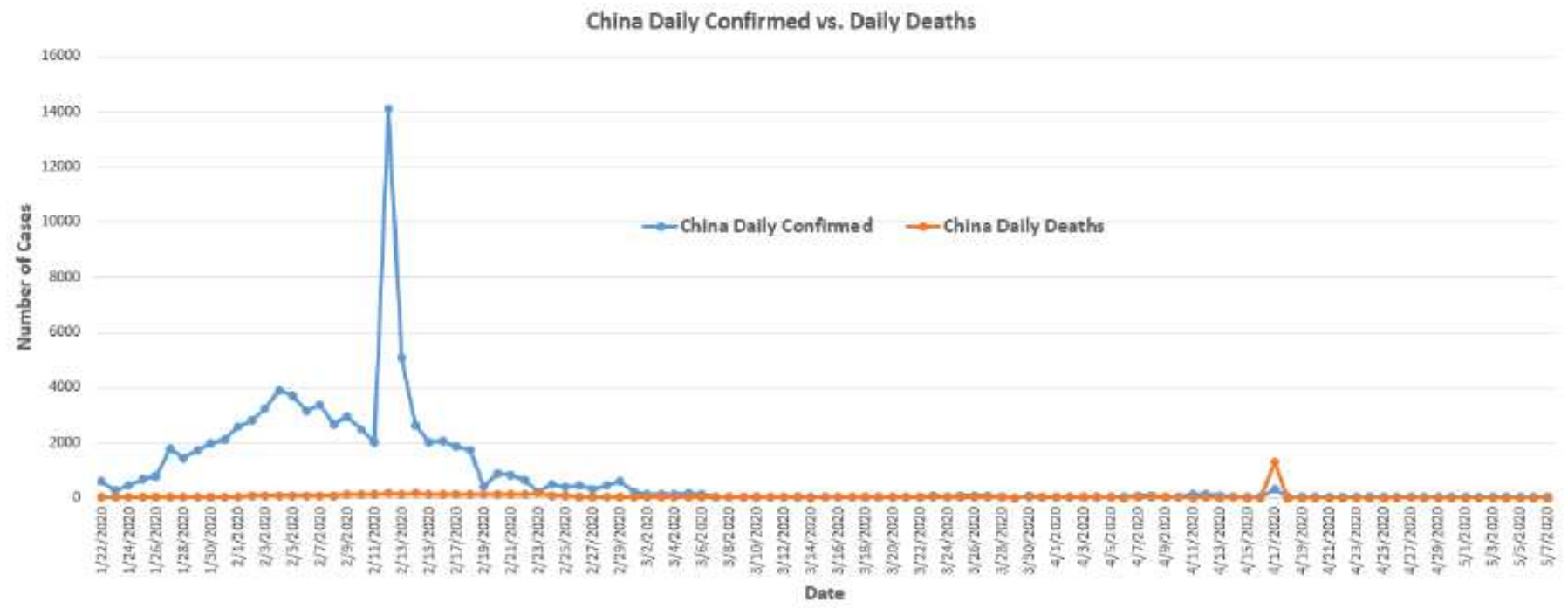

\section{Figure 2}

COVID19 - China Daily Infected Cases and Deaths, the date is from 1/22/2020 to 5/7/2020. 


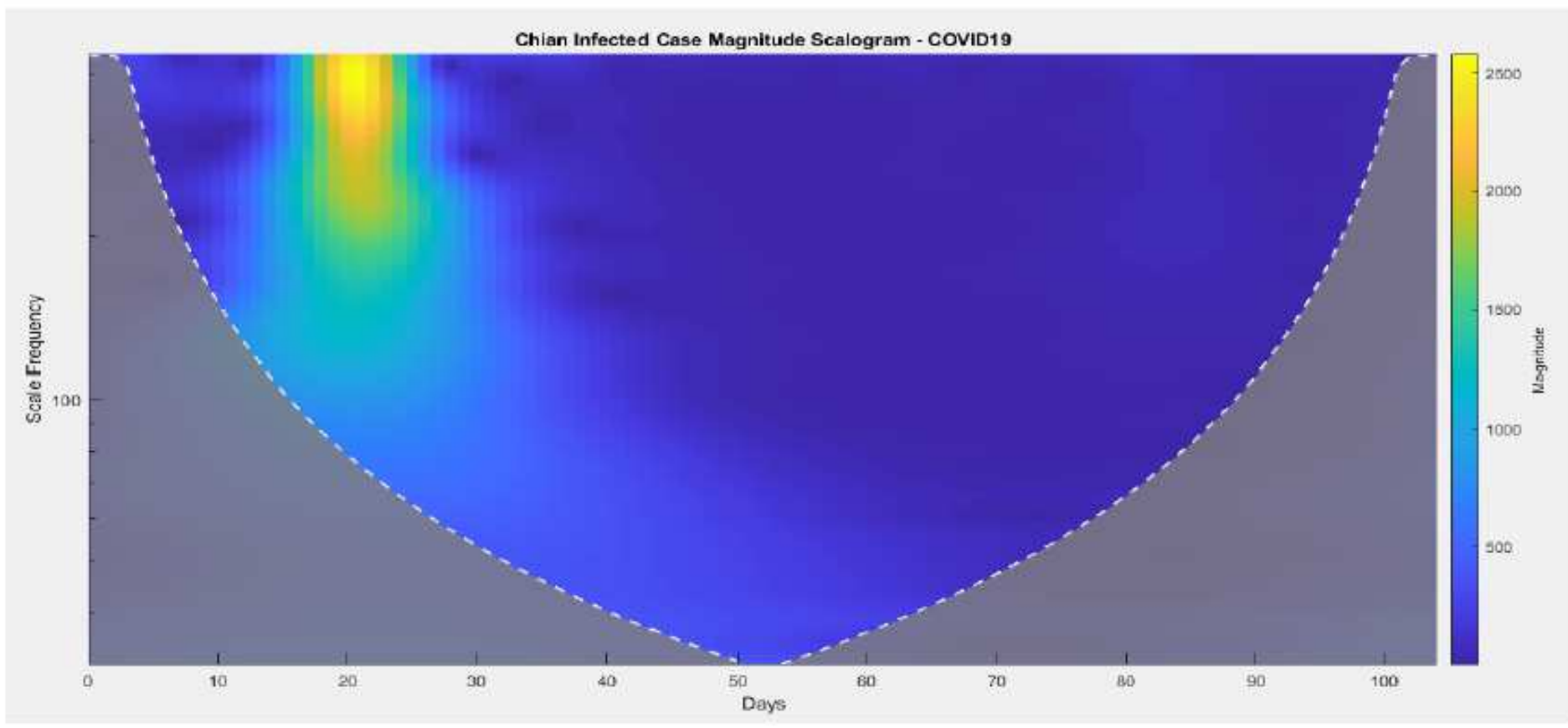

\section{Figure 3}

COVID19 - China Daily Infected Cases Wavelet Magnitude Scalogram, the date is from $1 / 22 / 2020$ to $5 / 7 / 2020$.

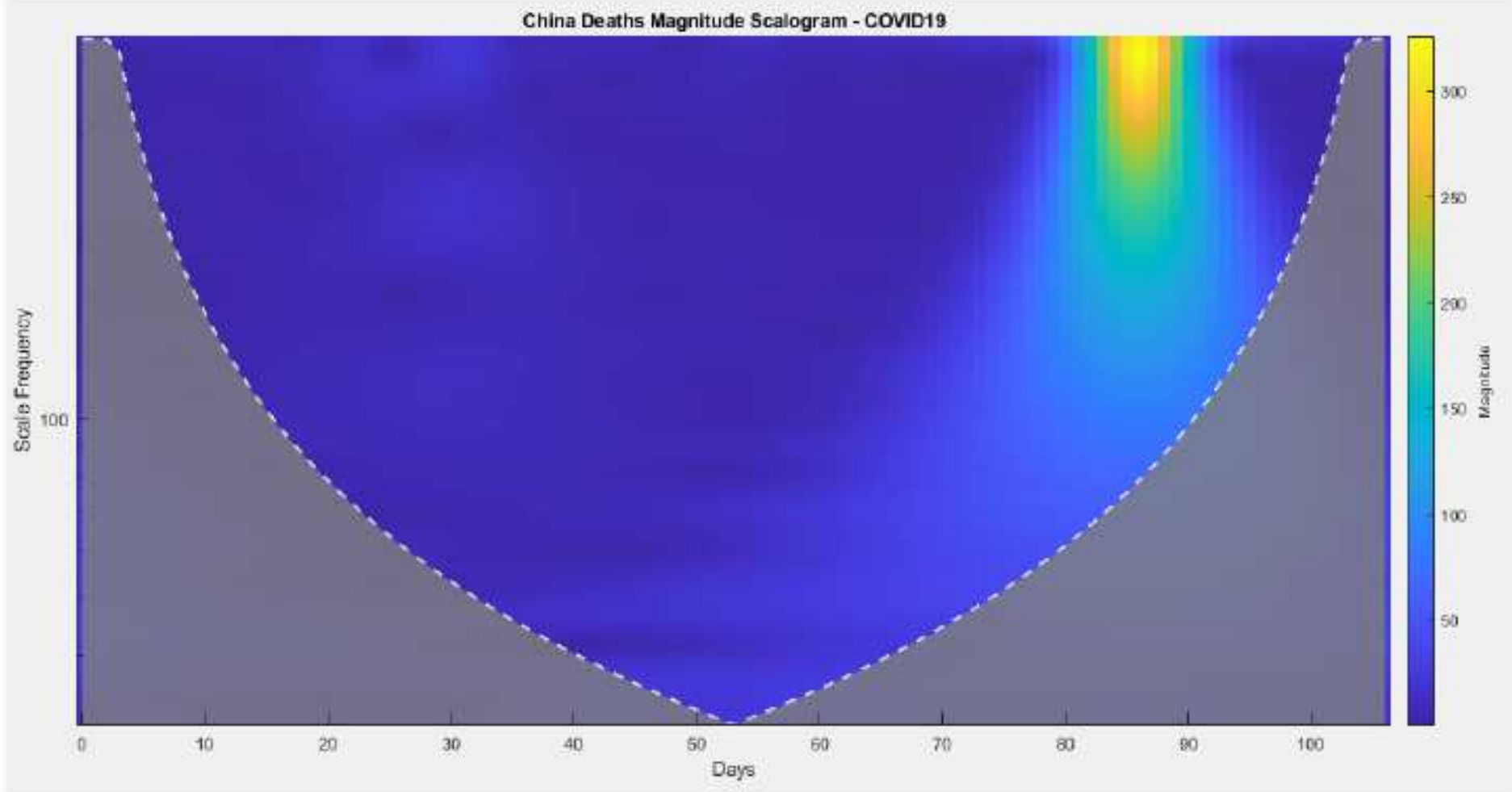

Figure 4

COVID19 - China Daily Deaths Wavelet Magnitude Scalogram, the date is from 1/22/2020 to 5/7/2020. 
U.S.A. Daily Confirmed Cases vs. Daily Deaths

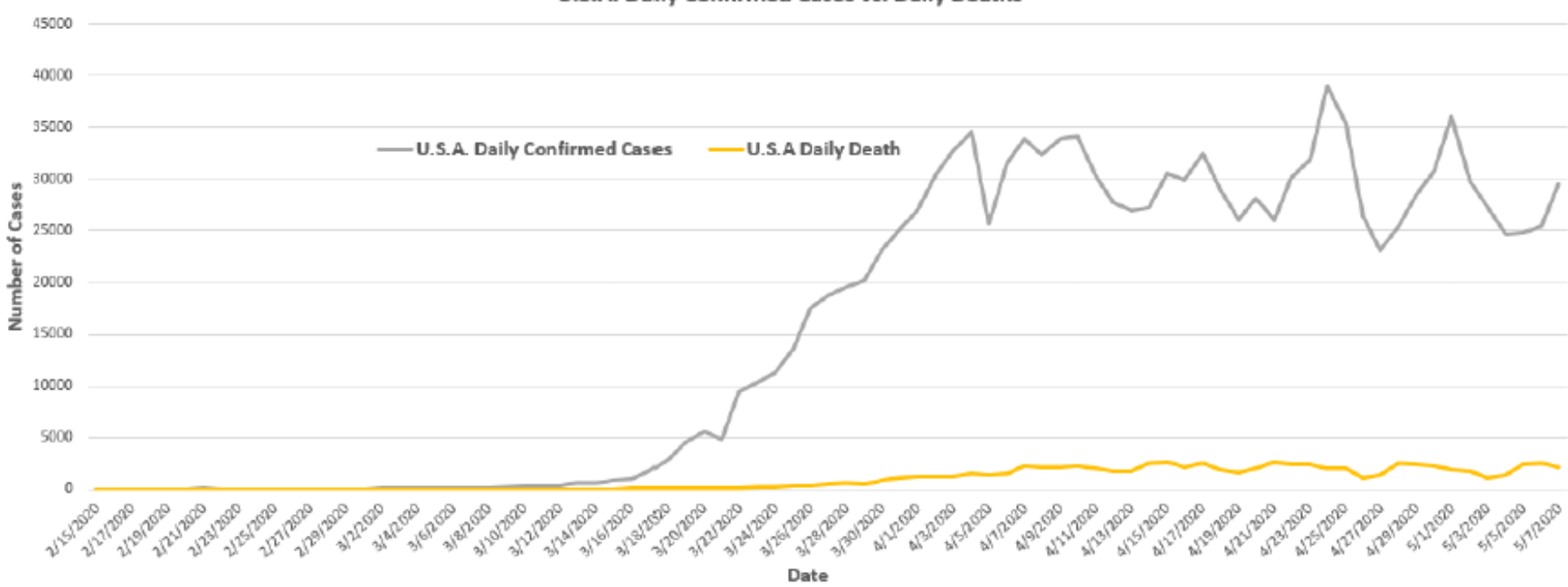

\section{Figure 5}

COVID19 - U.S.A Daily Infected Cases and Deaths, the date is from 2/15/2020 to 5/7/2020.

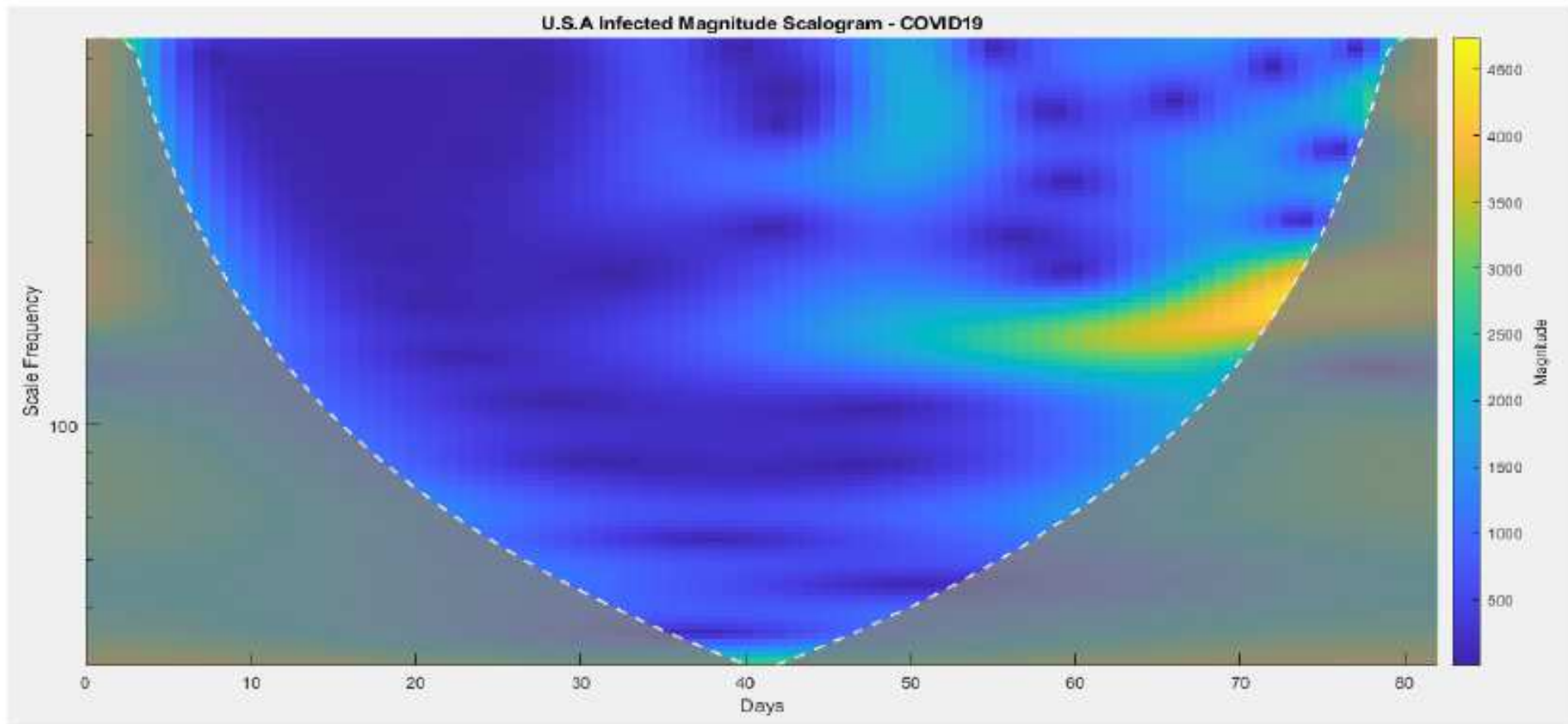

\section{Figure 6}

COVID19 - U.S.A Daily Infected Cases Wavelet Magnitude Scalogram, the date is from 2/15/2020 to $5 / 7 / 2020$ 


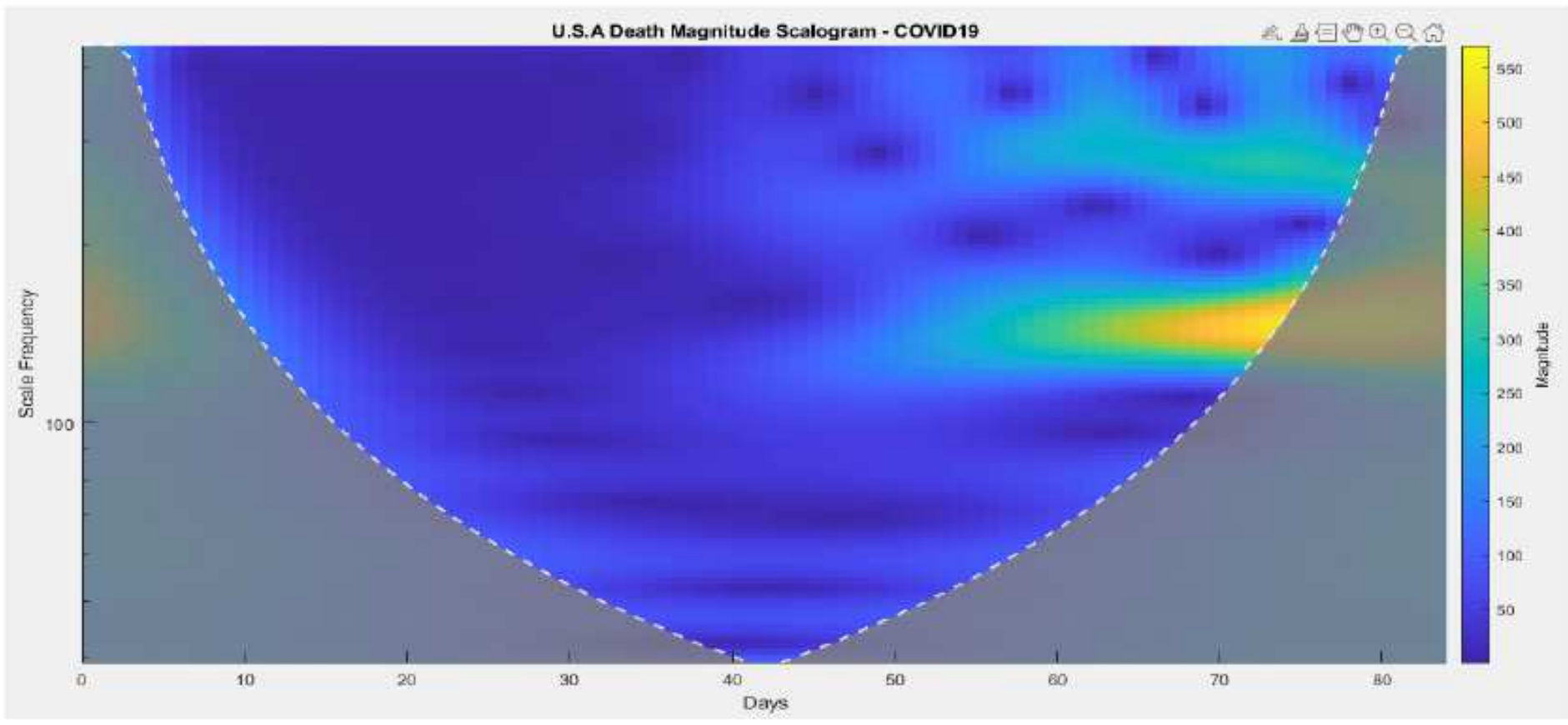

\section{Figure 7}

COVID19 - USA Daily Deaths Wavelet Magnitude Scalogram, the date is from 2/15/2020 to 5/7/2020.

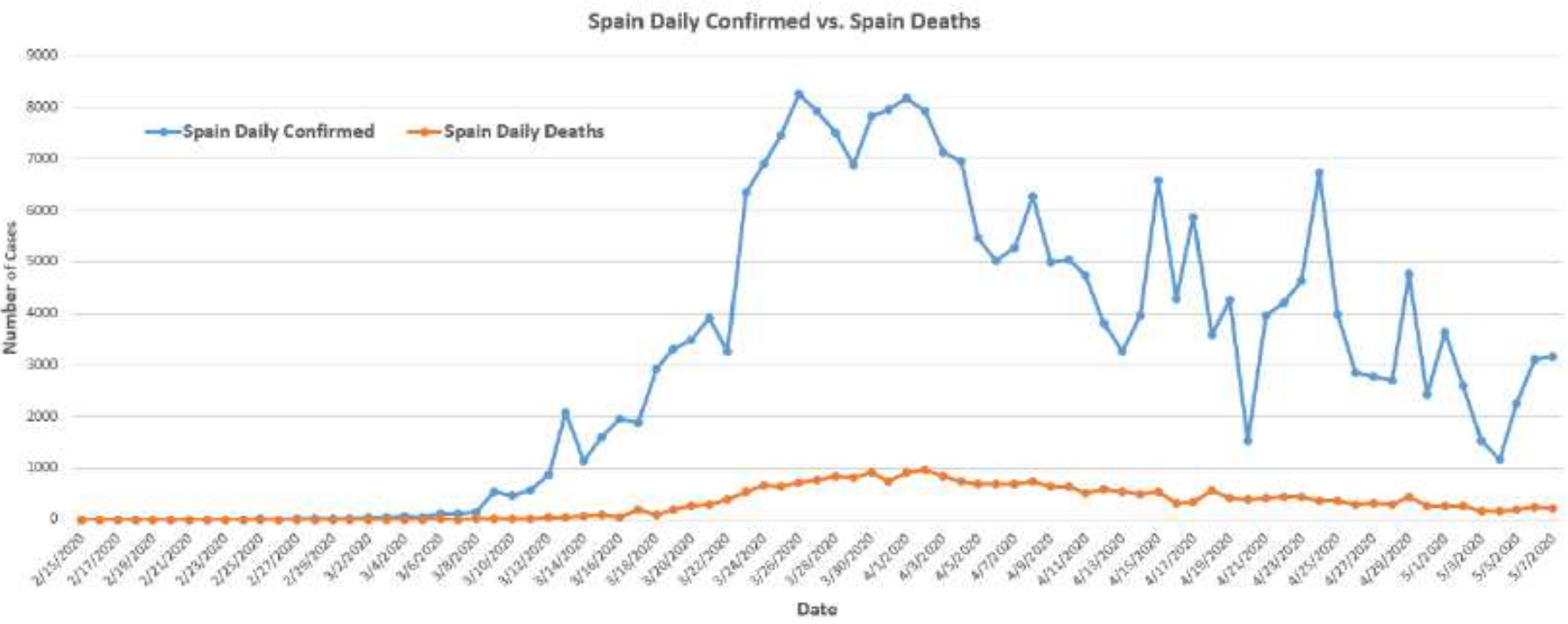

Figure 8

COVID19 - Spain Daily Infected Cases and Deaths, the date is from 2/15/2020 to 5/7/2020. 


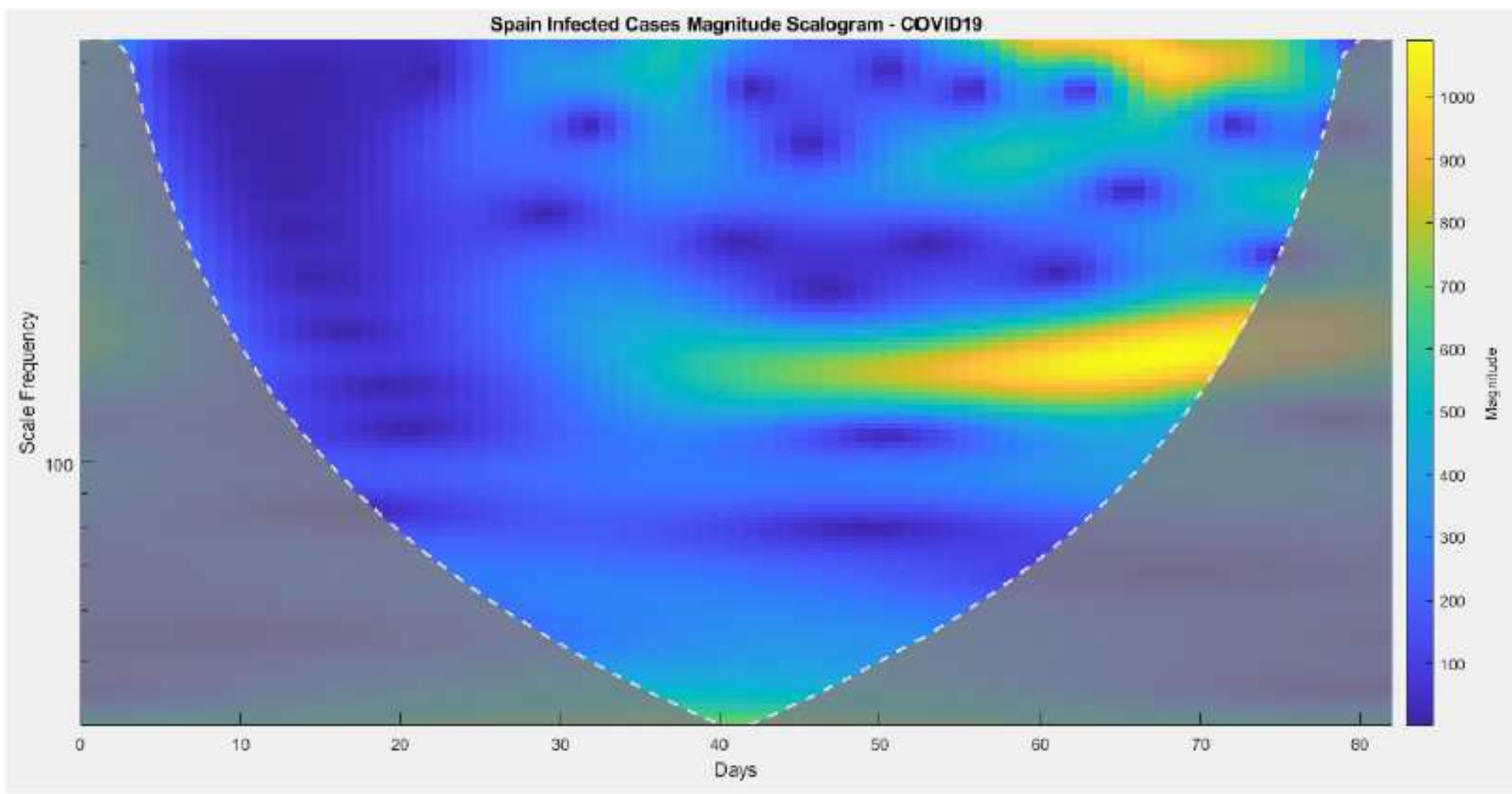

\section{Figure 9}

COVID19 - Spain Daily Infected Cases Wavelet Magnitude Scalogram, the date is from 2/15/2020 to $5 / 7 / 2020$

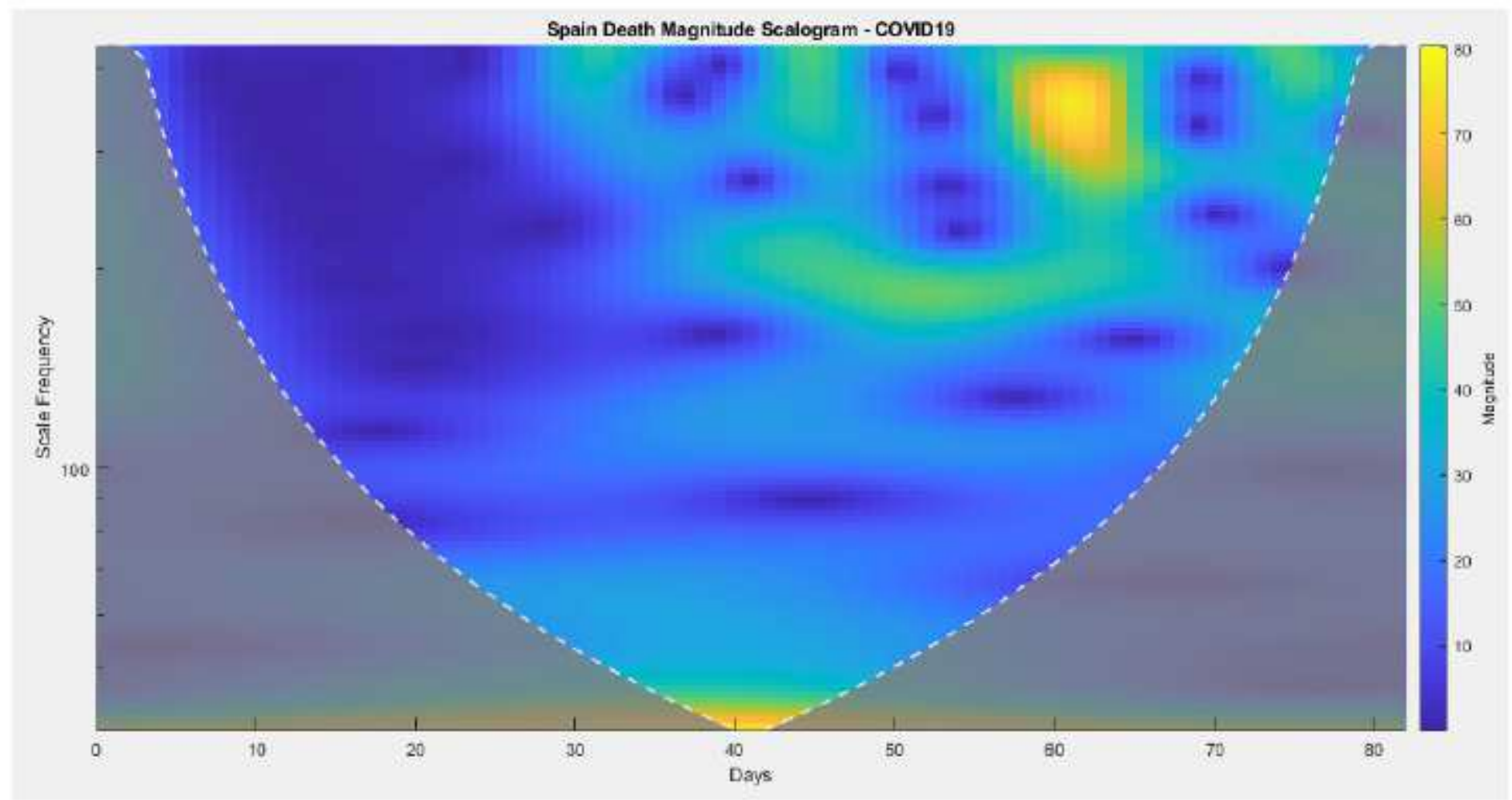

Figure 10 
COVID19 - Spain Daily Deaths Wavelet Magnitude Scalogram, the date is from 2/15/2020 to 5/7/2020.

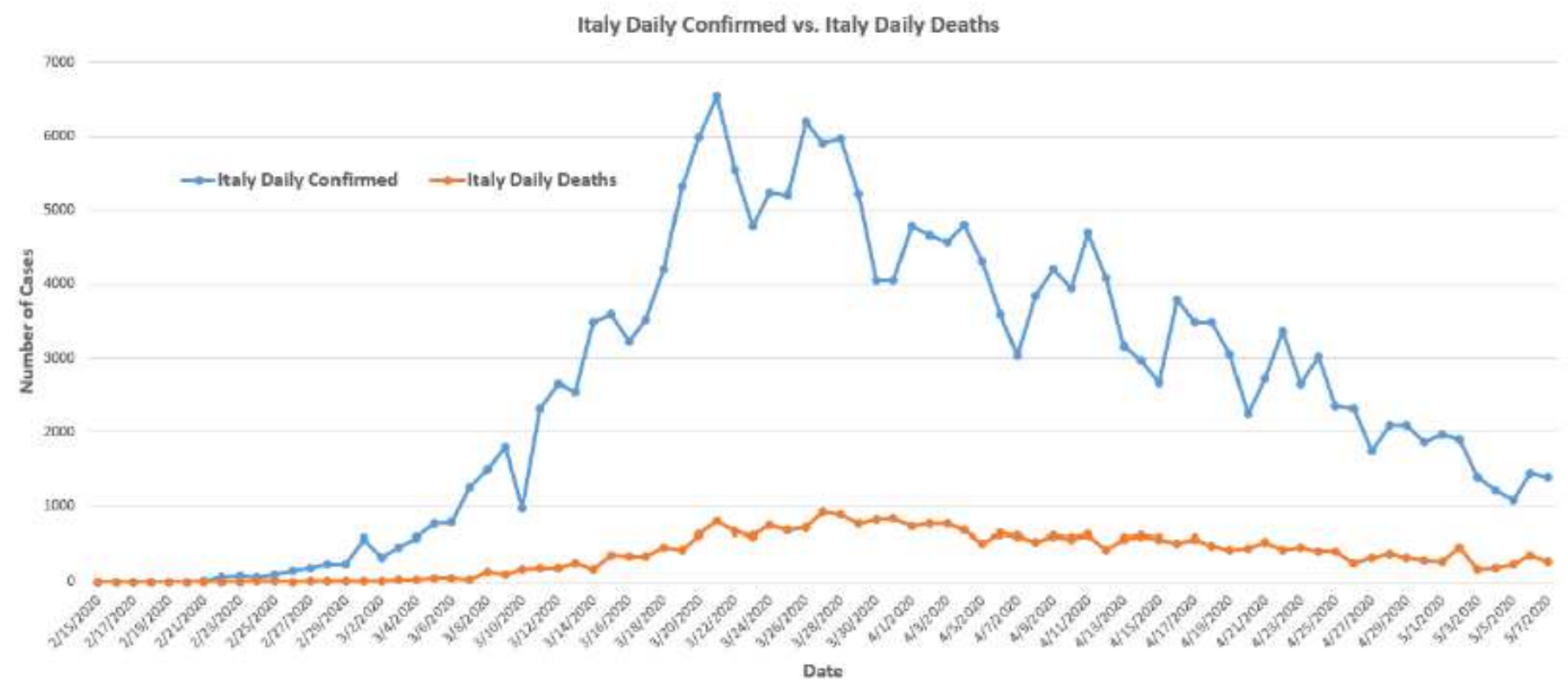

\section{Figure 11}

COVID19 - Italy Daily Infected Cases and Deaths, the date is from 2/15/2020 to 5/7/2020.

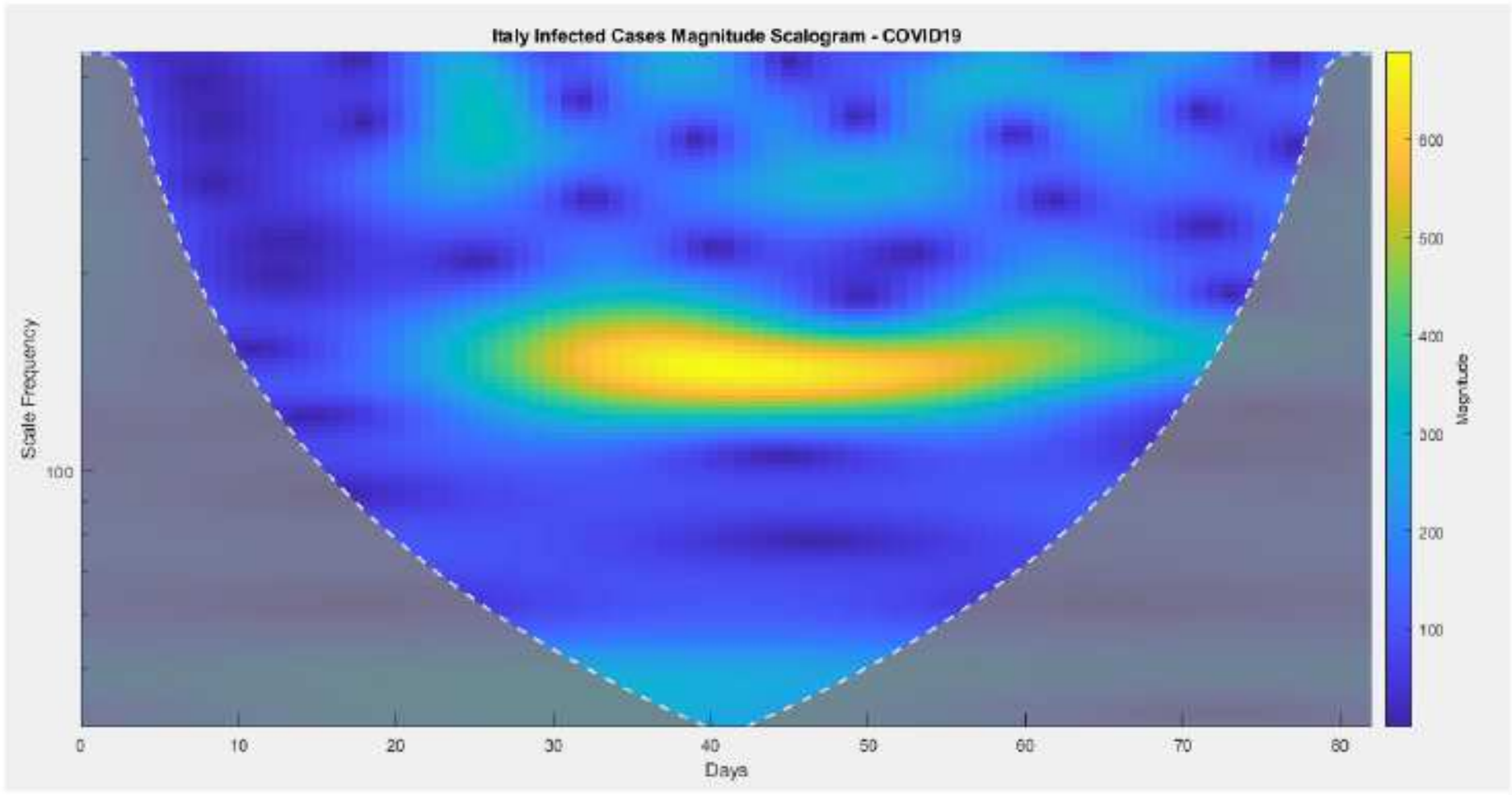

Figure 12 
COVID19 - Italy Daily Infected Cases Wavelet Magnitude Scalogram, the date is from 2/15/2020 to $5 / 7 / 2020$

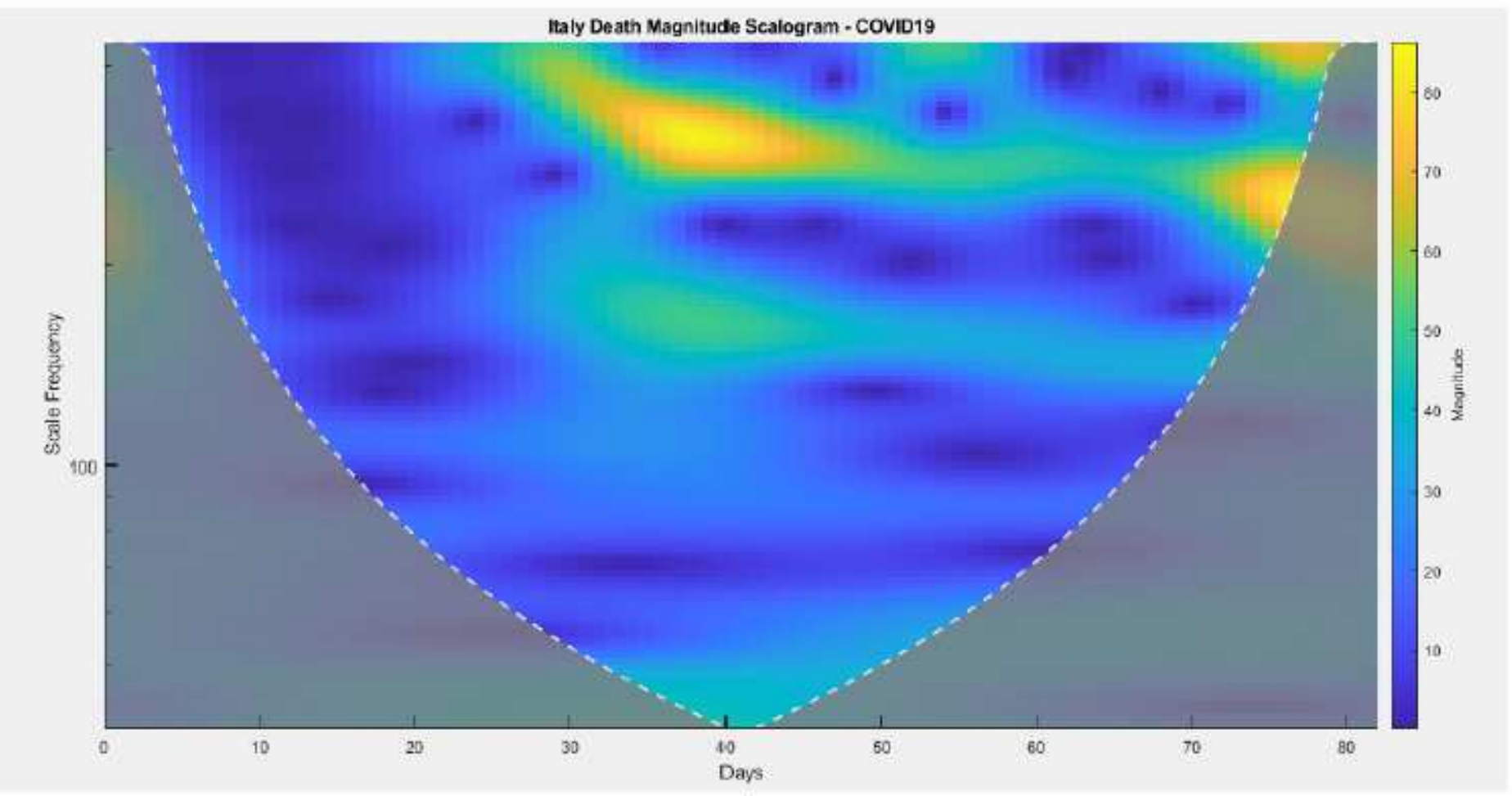

Figure 13

COVID19 - Italy Daily Deaths Wavelet Magnitude Scalogram, the date is from 2/15/2020 to 5/7/2020.

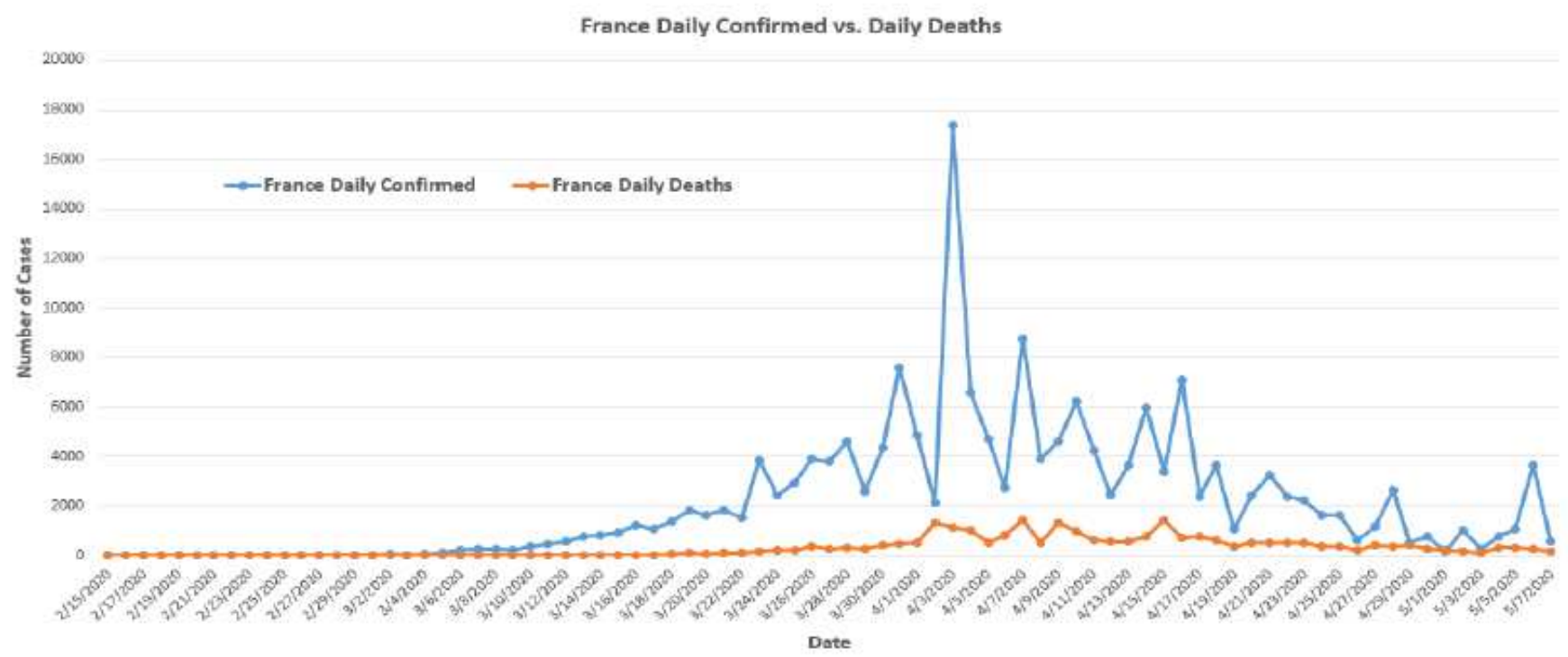

Figure 14

COVID19 - France Daily Infected Cases and Deaths, the date is from 2/15/2020 to 5/7/2020. 


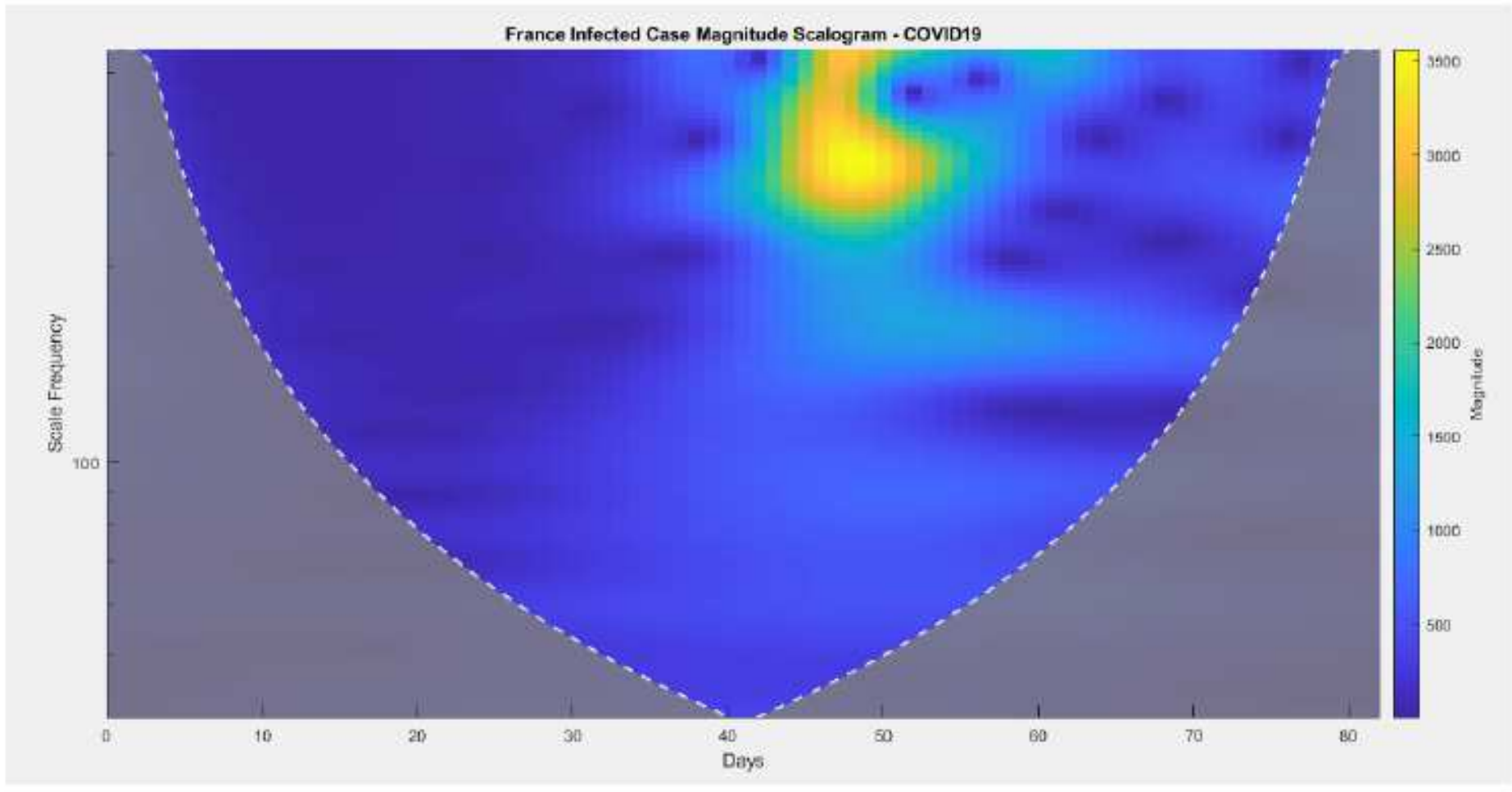

Figure 15

COVID19 - France Daily Infected Cases Wavelet Magnitude Scalogram, the date is from 2/15/2020 to $5 / 7 / 2020$

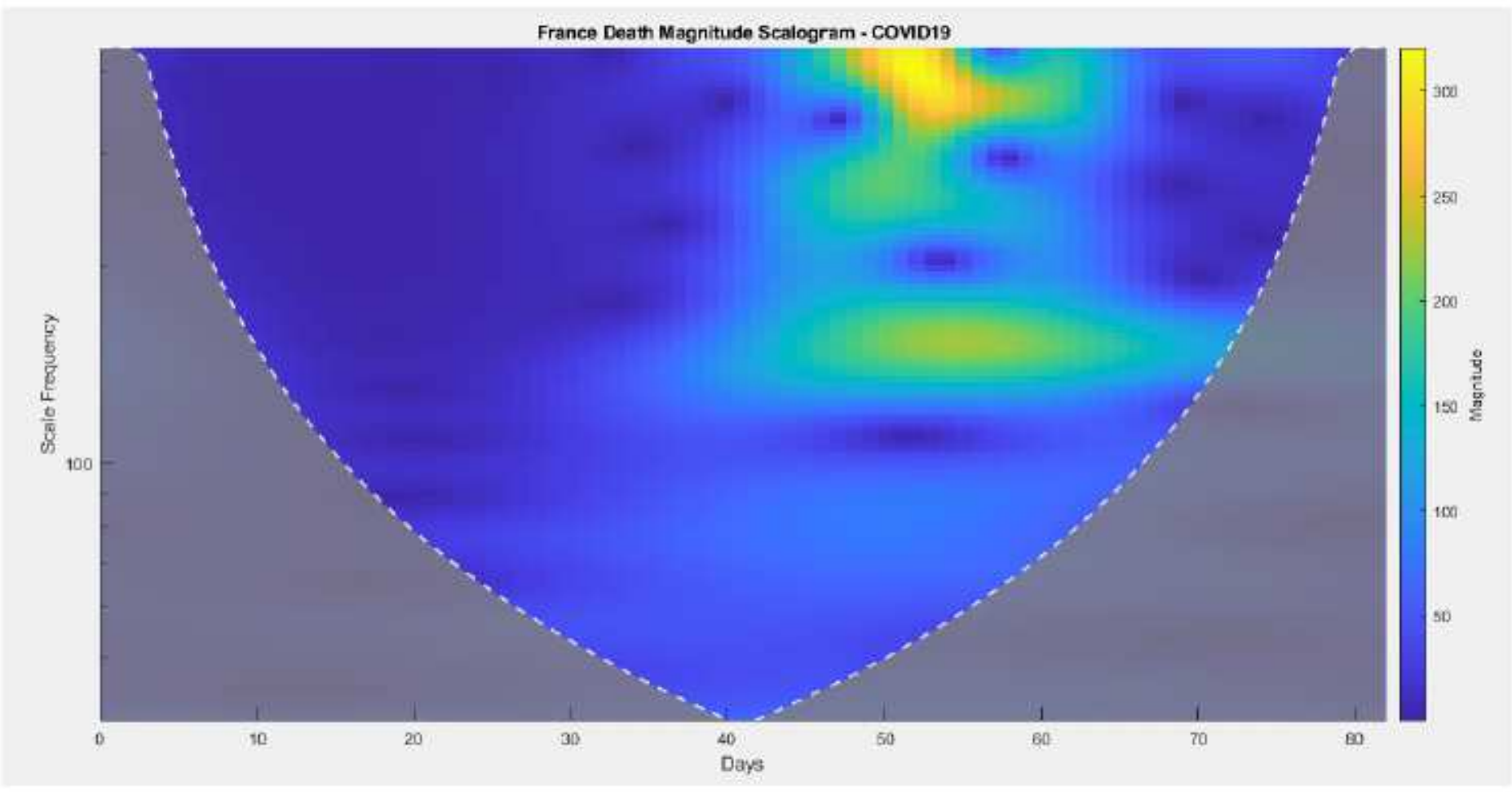

Figure 16 
COVID19 - France Daily Deaths Wavelet Magnitude Scalogram, the date is from 2/15/2020 to 5/7/2020.

Germany Daily Confirmed vs. Daily Deaths

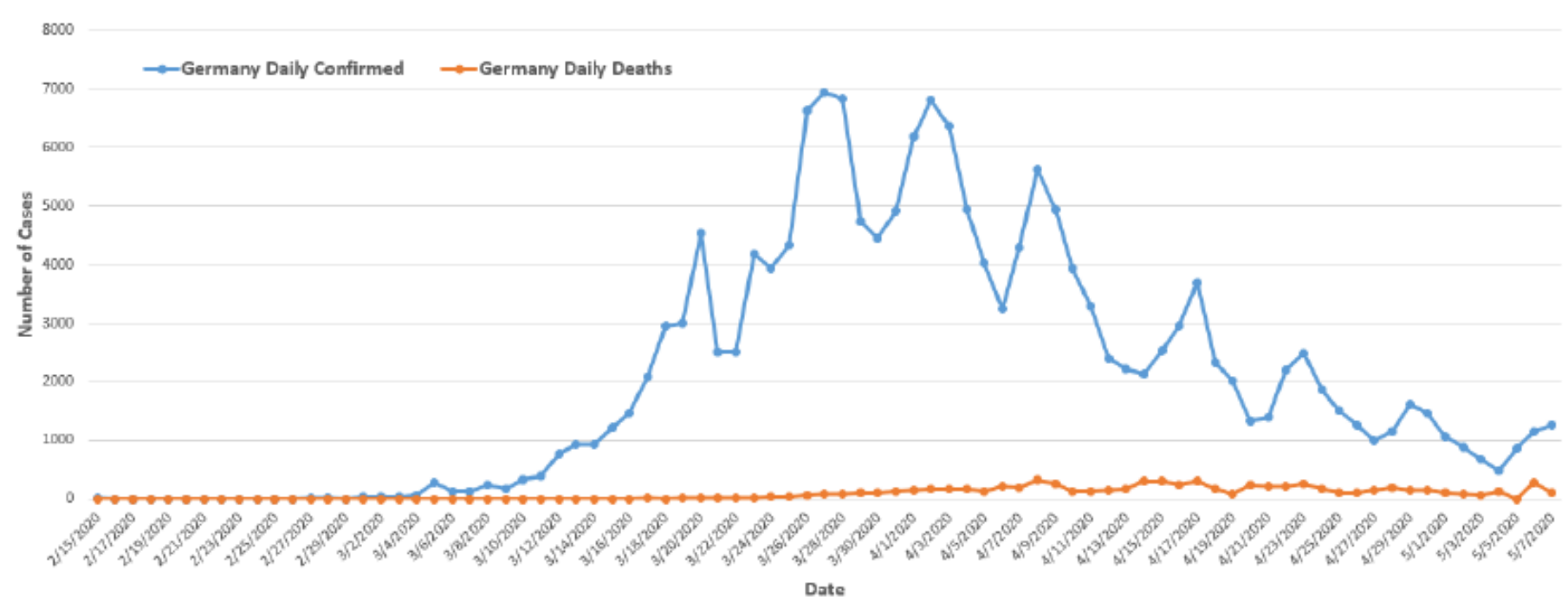

Figure 17

COVID19 - Germany Daily Infected Cases and Deaths, the date is from 2/15/2020 to 5/7/2020.

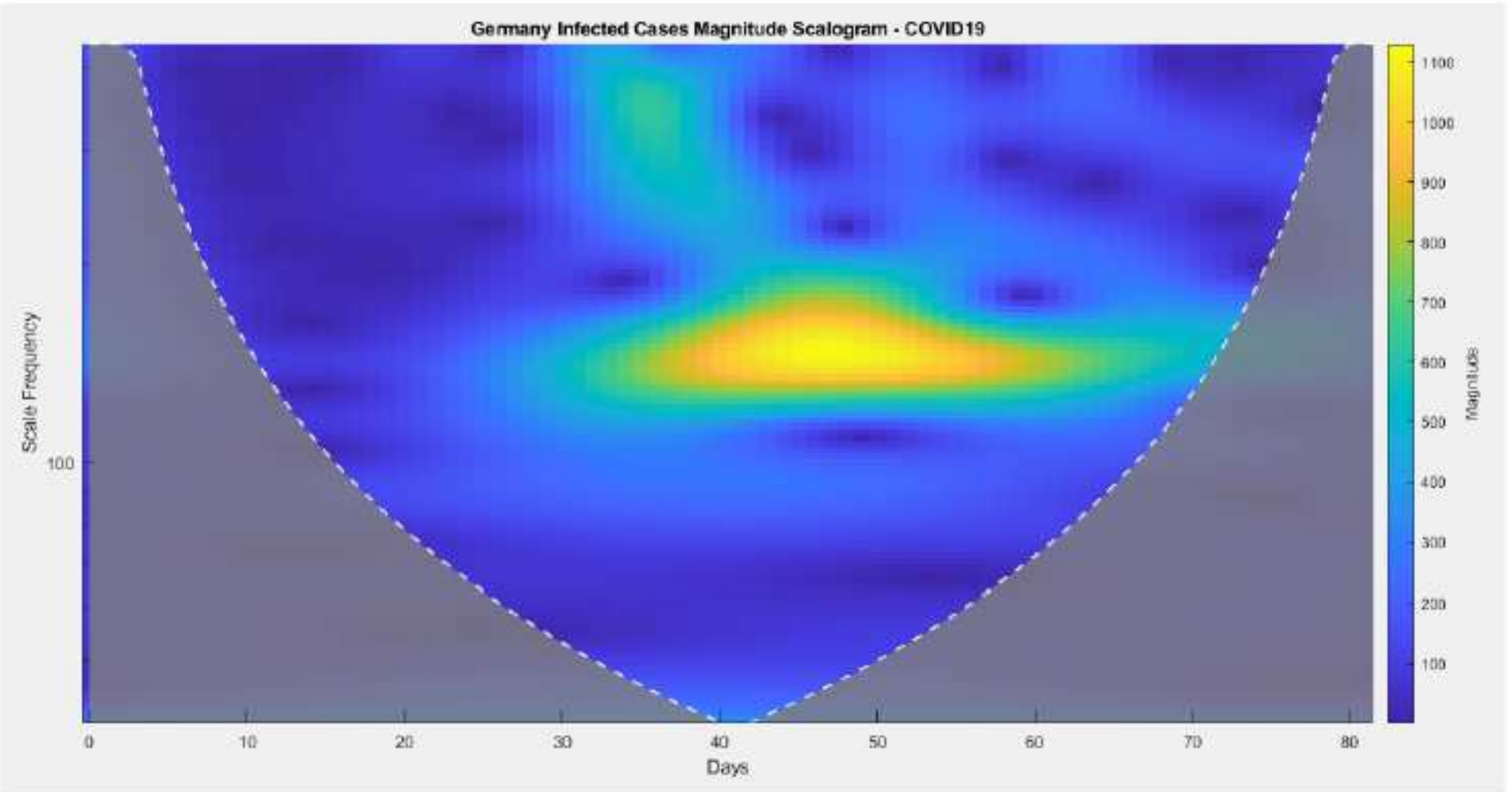

Figure 18

COVID19 - Germany Daily Infected Cases Wavelet Magnitude Scalogram, the date is from 2/15/2020 to $5 / 7 / 2020$ 


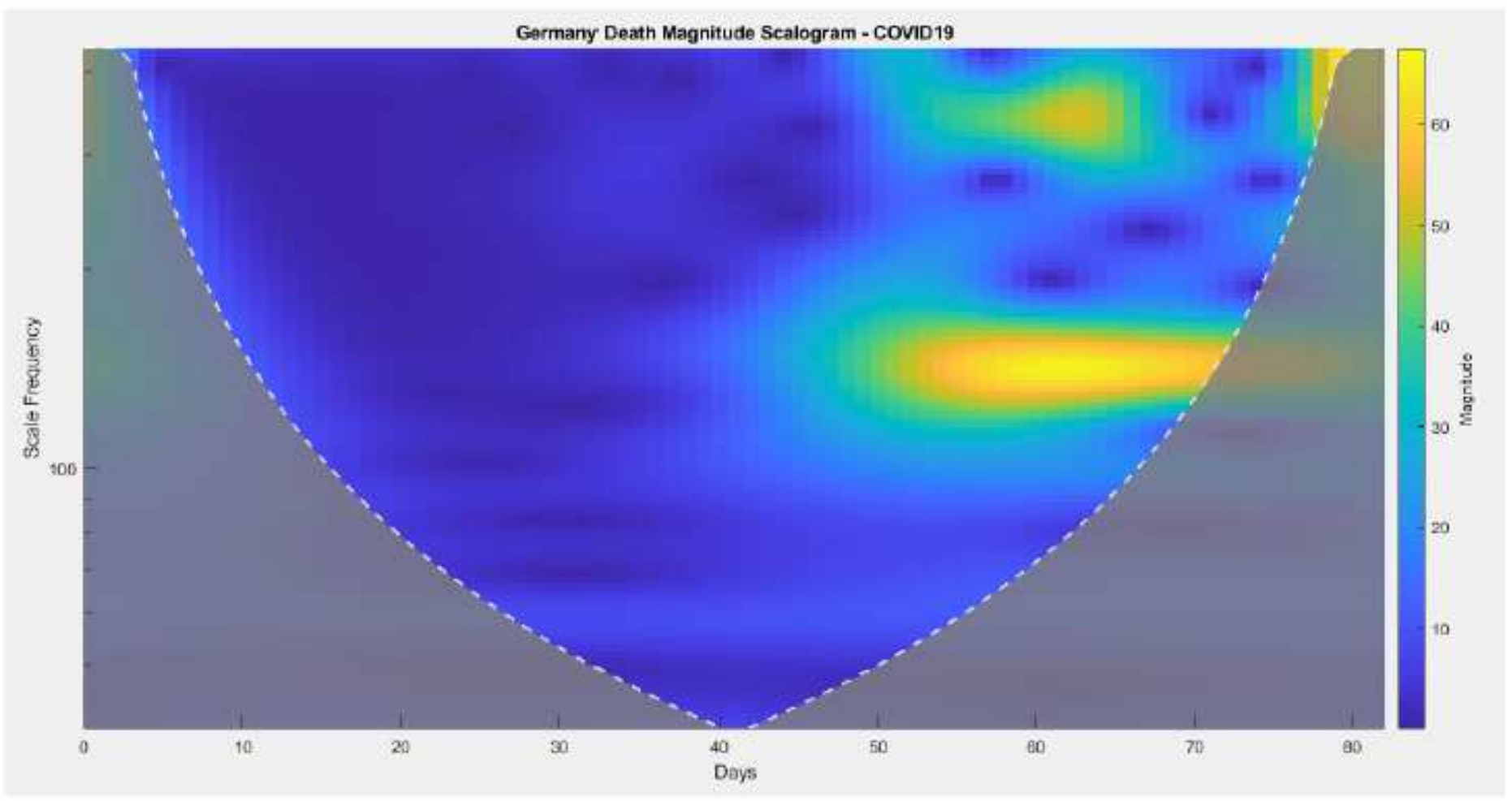

Figure 19

COVID19 - Germany Daily Deaths Wavelet Magnitude Scalogram, the date is from 2/15/2020 to $5 / 7 / 2020$.

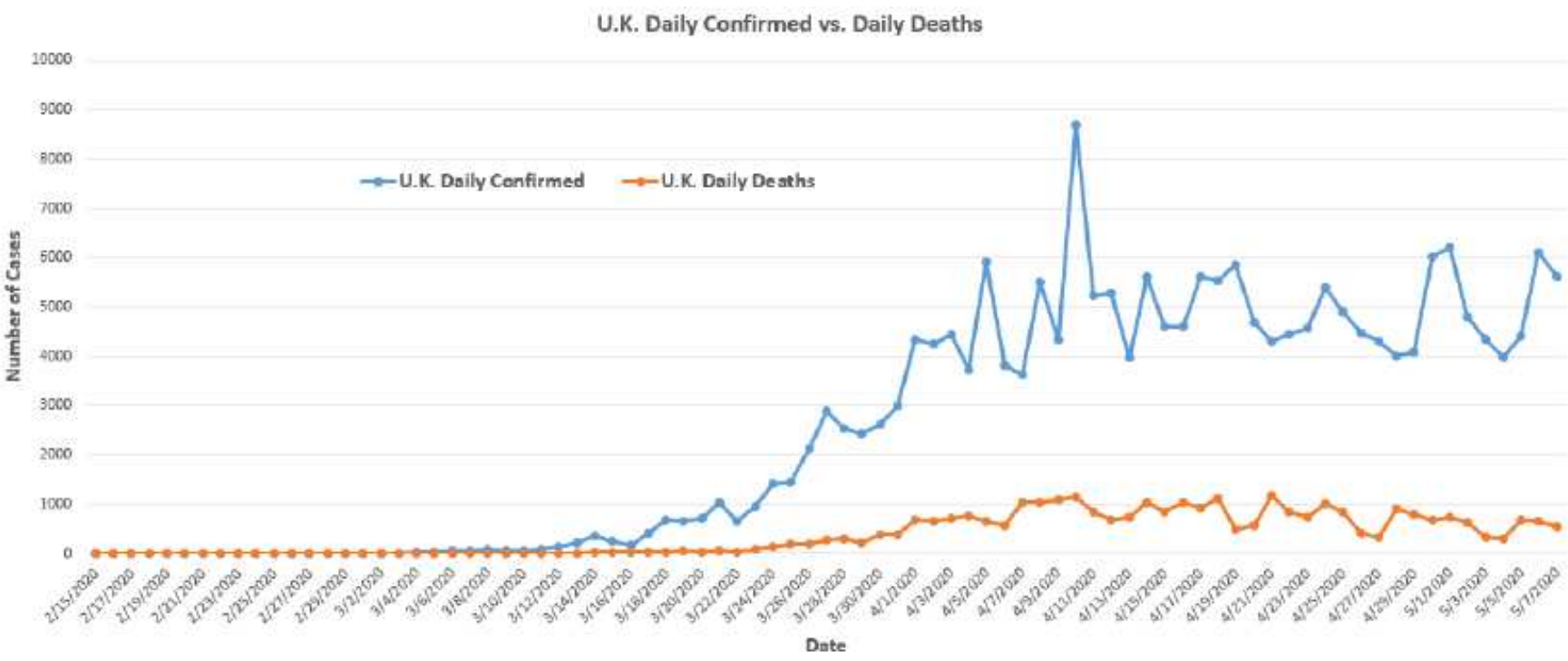

Figure 20

COVID19 - U.K. Daily Infected Cases and Deaths, the date is from 2/15/2020 to 5/7/2020. 


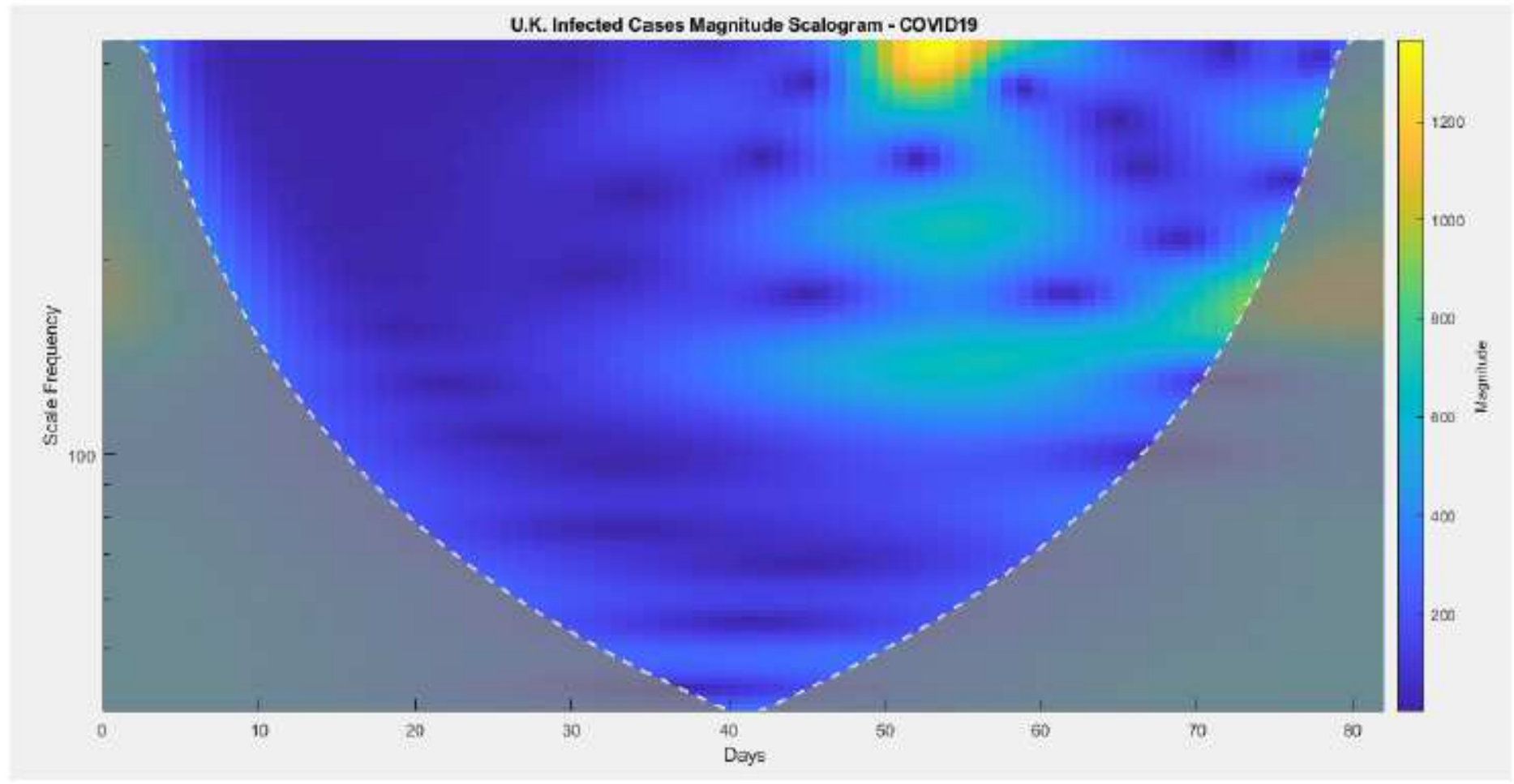

\section{Figure 21}

COVID19 - U.K. Daily Infected Cases Wavelet Magnitude Scalogram, the date is from $2 / 15 / 2020$ to $5 / 7 / 2020$

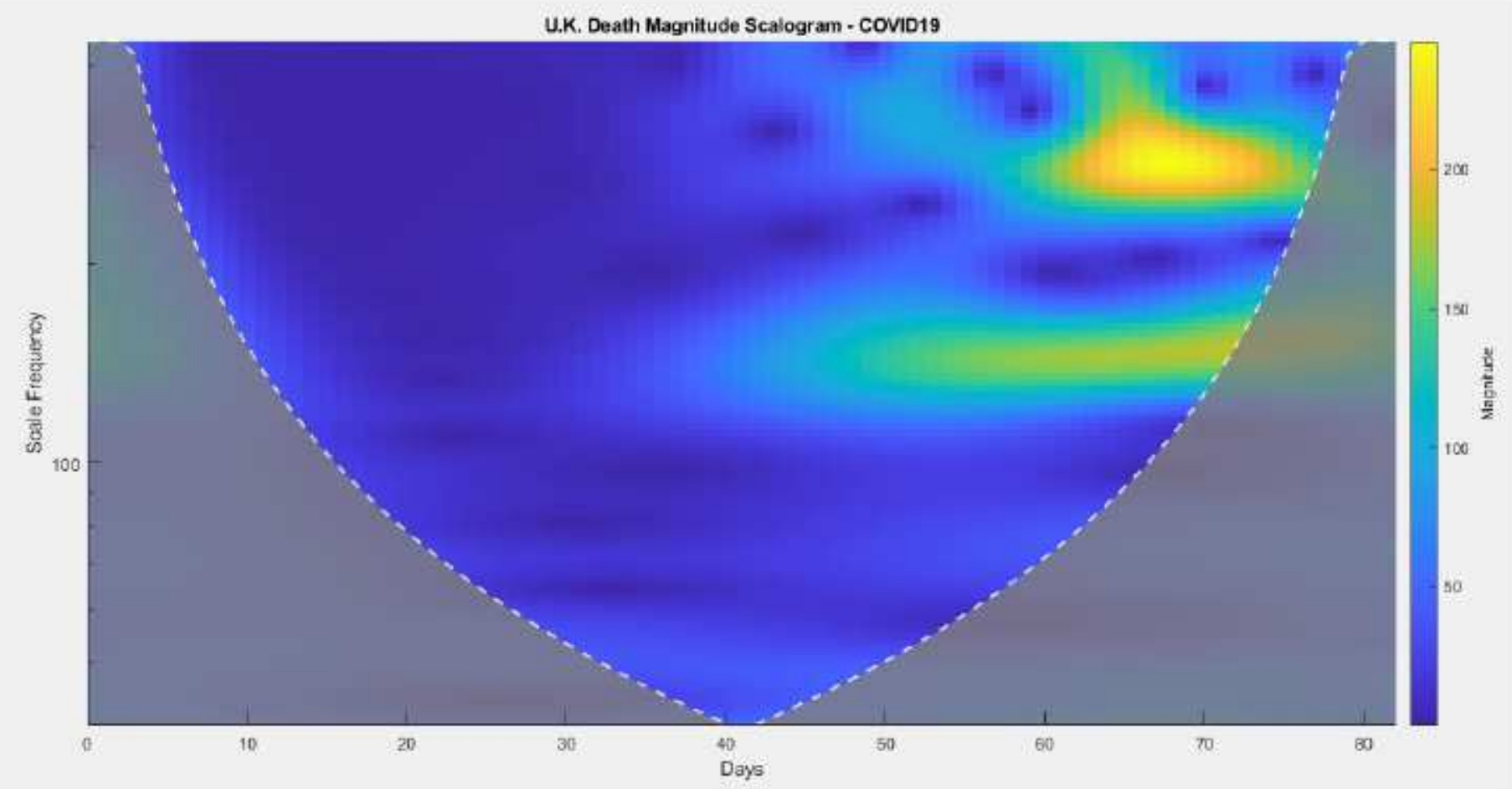

Figure 22 
COVID19 - U.K. Daily Deaths Wavelet Magnitude Scalogram, the date is from 2/15/2020 to 5/7/2020.

S. Korea Daily Confirmed vs. Daily Deaths

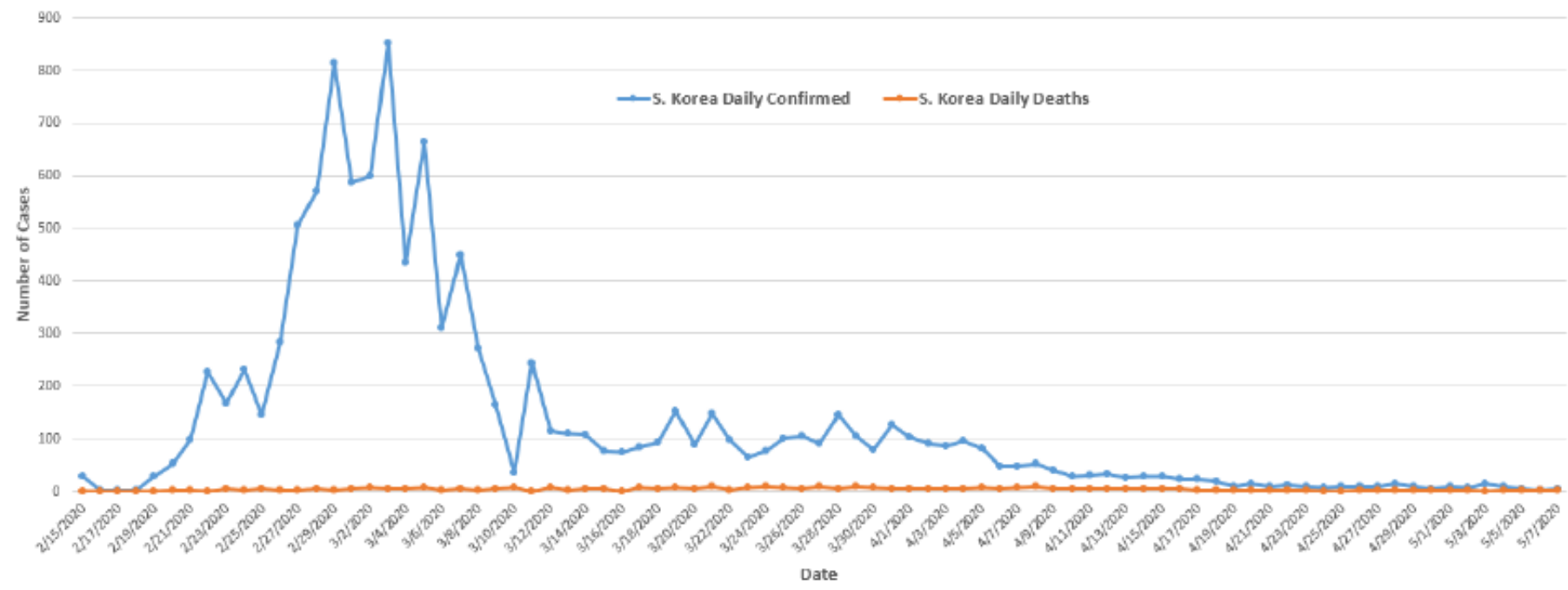

Figure 23

COVID19 - South Korea Daily Infected Cases and Deaths, the date is from 2/15/2020 to 5/7/2020.

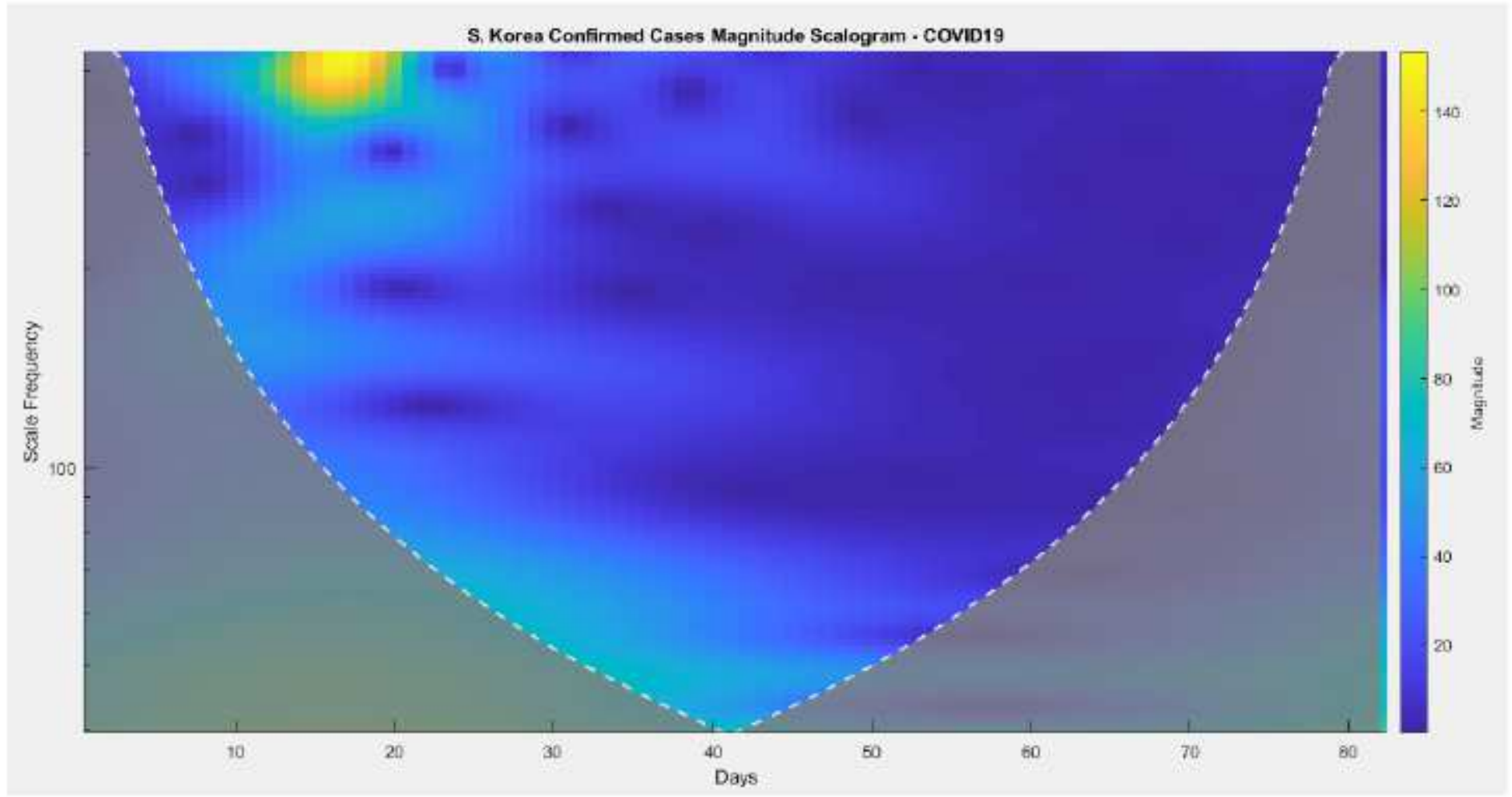

Figure 24

COVID19 - South Korea Daily Infected Cases Wavelet Magnitude Scalogram, the date is from 2/15/2020 to $5 / 7 / 2020$ 


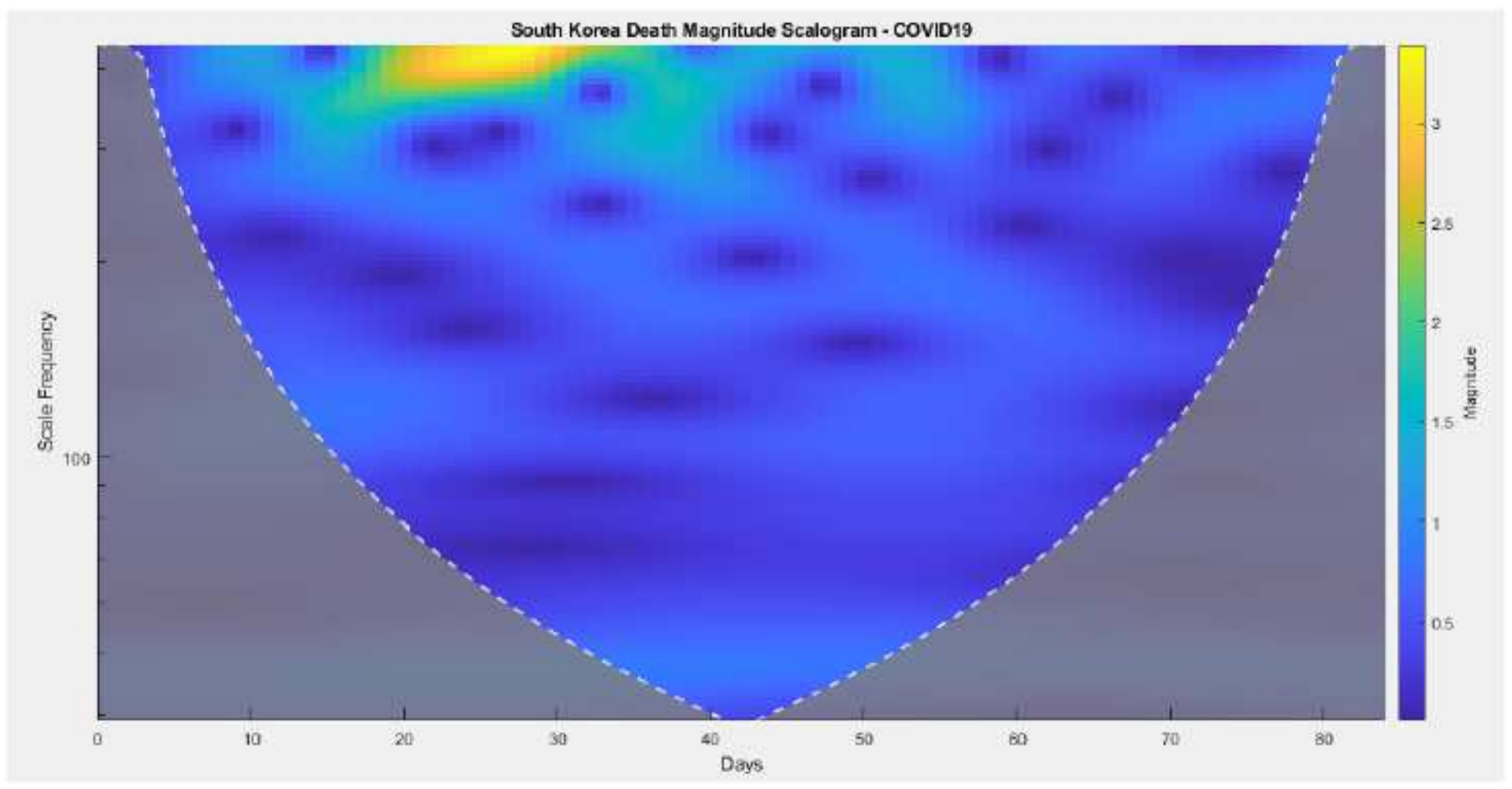

\section{Figure 25}

COVID19 - South Korea Daily Deaths Wavelet Magnitude Scalogram, the date is from 2/15/2020 to $5 / 7 / 2020$.

\section{Michigan Daily Confirmed vs Daily Deaths}

2500

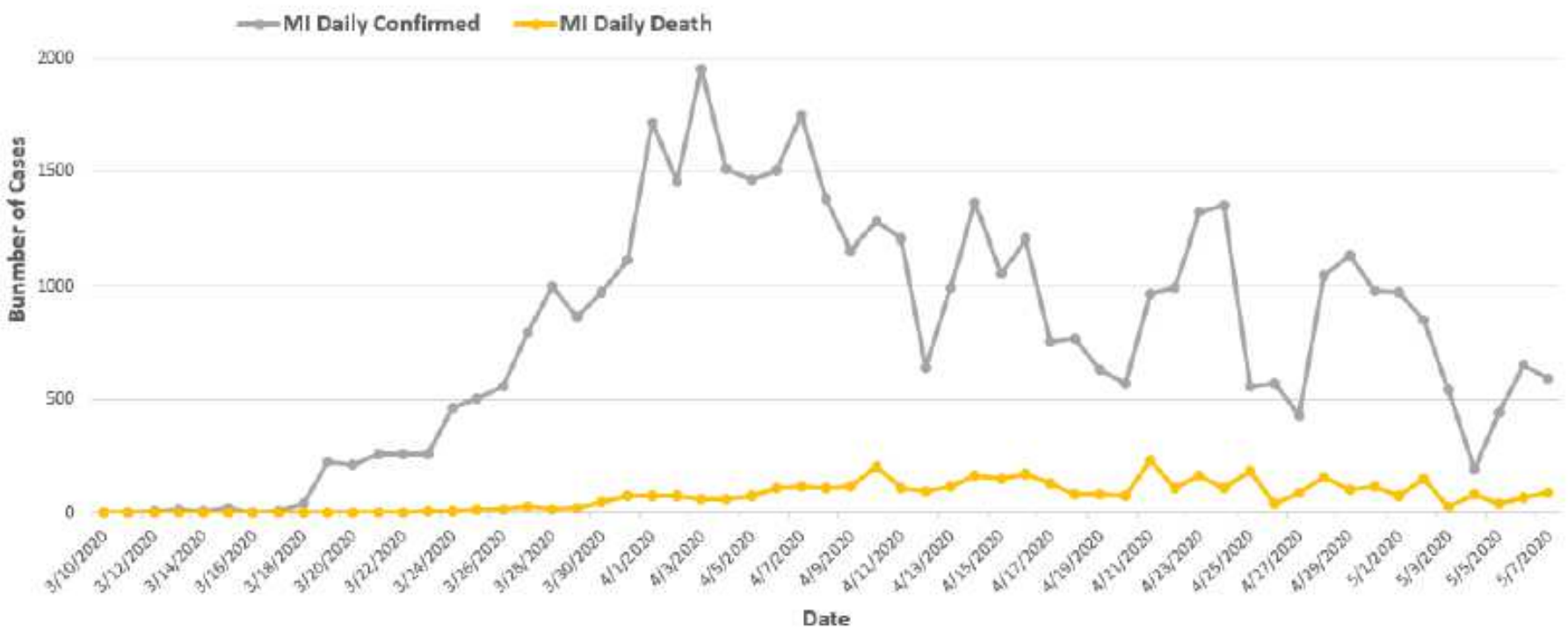

Figure 26

COVID19 - Michigan Daily Infected Cases and Deaths, the date is from 3/10/2020 to 5/7/2020. 


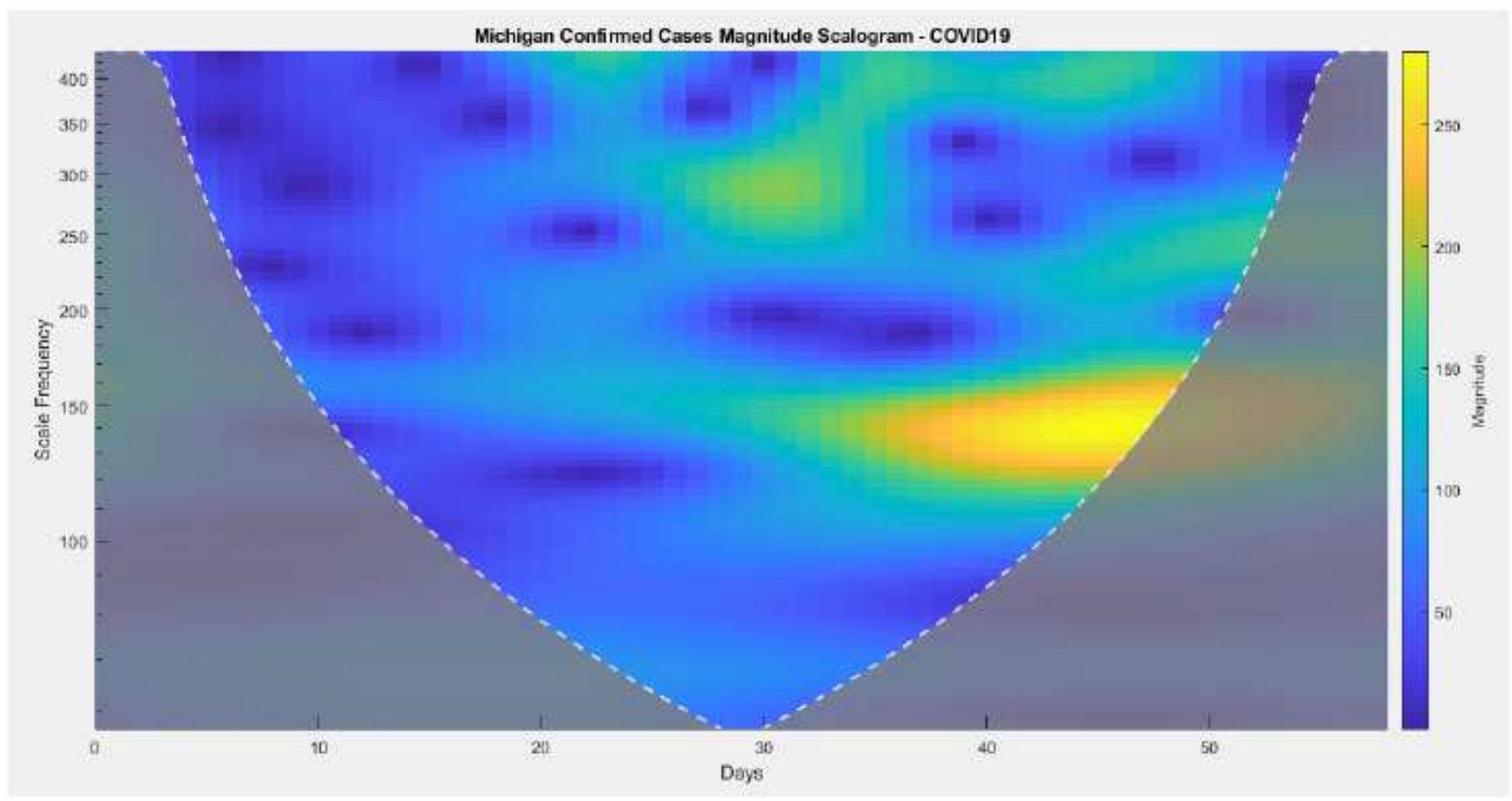

Figure 27

COVID19 - Michigan Daily Infected Cases Wavelet Magnitude Scalogram, the date is from 3/10/2020 to $5 / 7 / 2020$

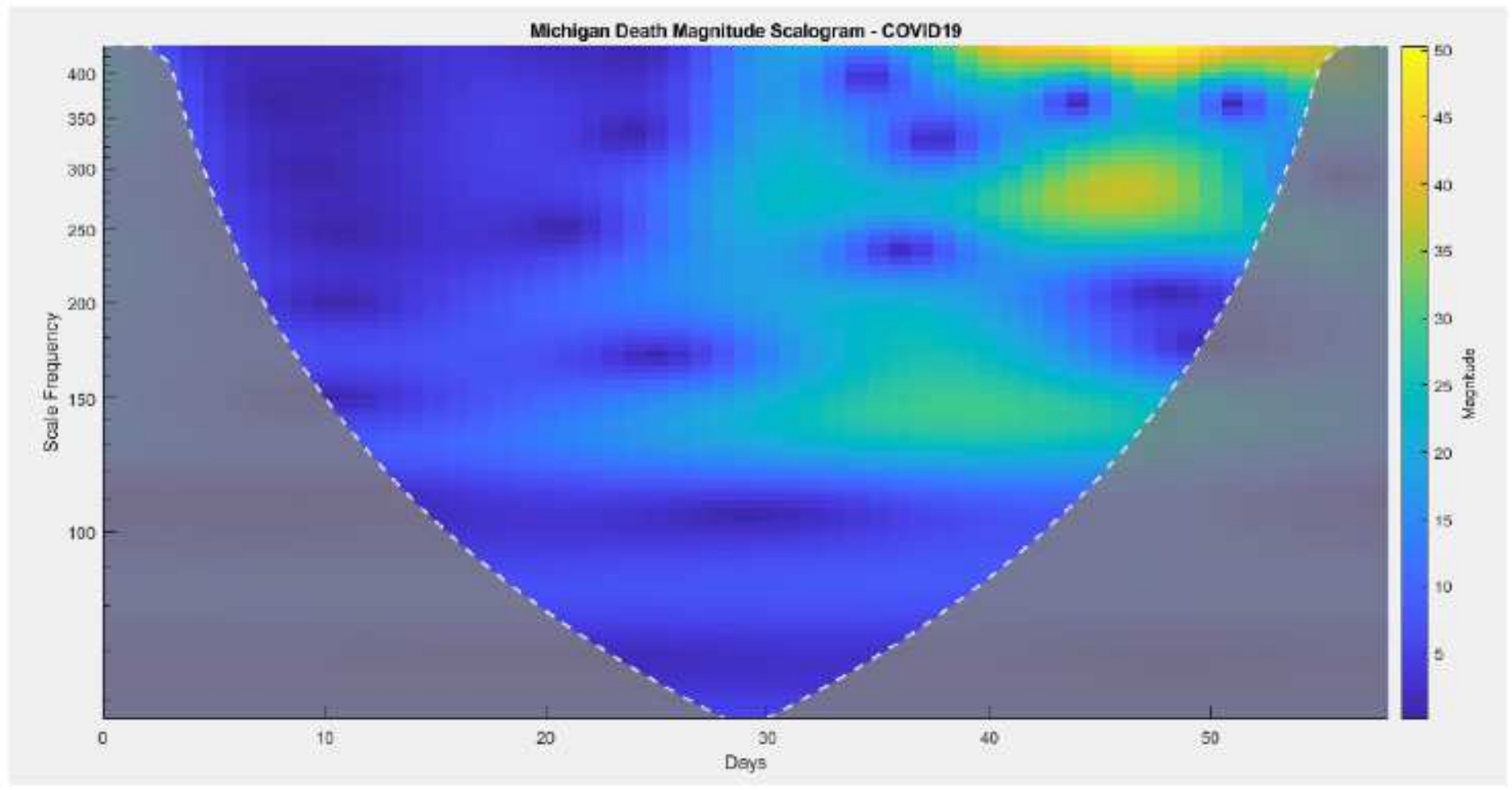


Figure 28

COVID19 - Michigan Daily Deaths Wavelet Magnitude Scalogram, the date is from 3/10/2020 to $5 / 7 / 2020$.

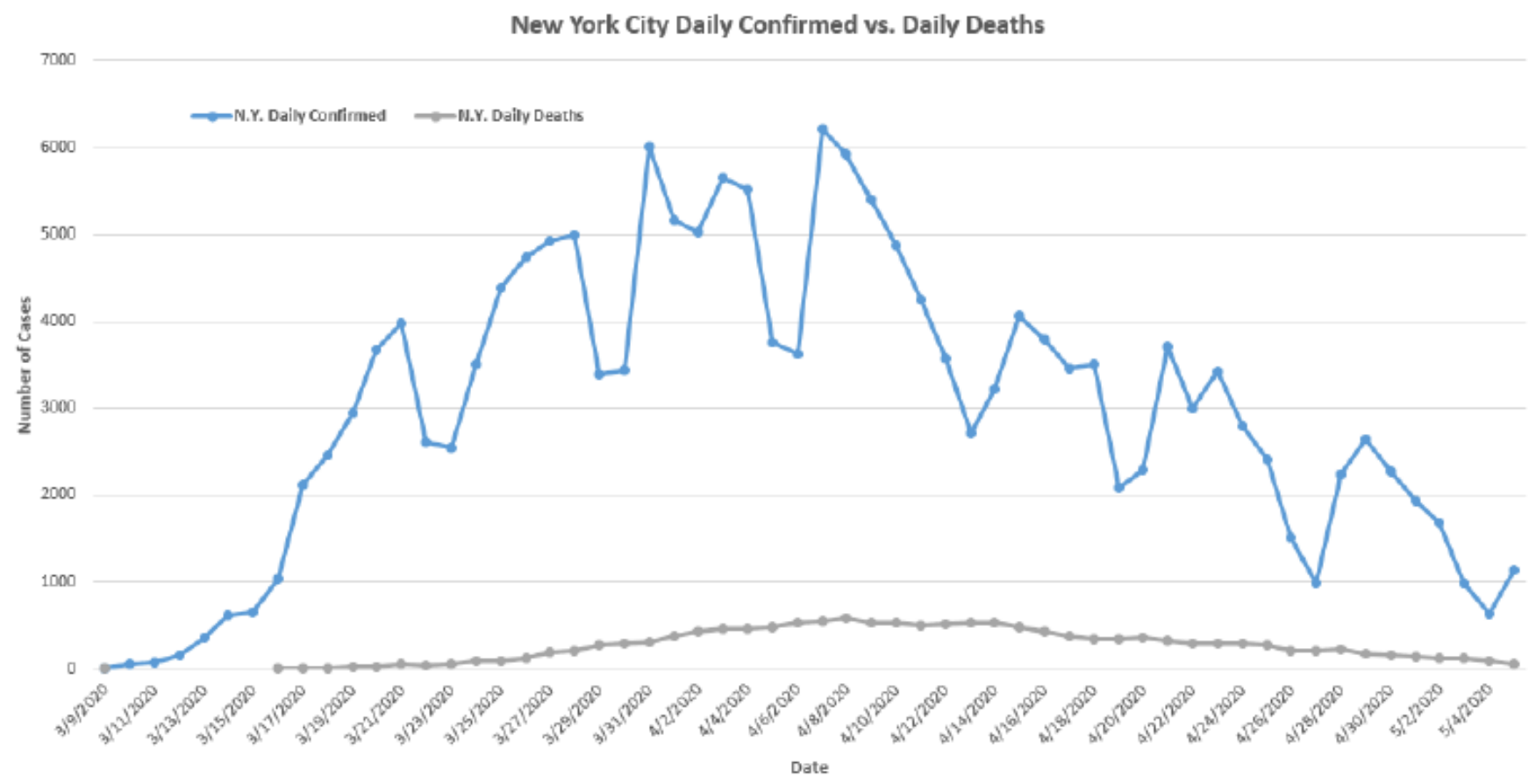

Figure 29

COVID 19 - New York City Daily Infected Cases and Deaths, the date is from 3/9/2020 to 5/5/2020. 


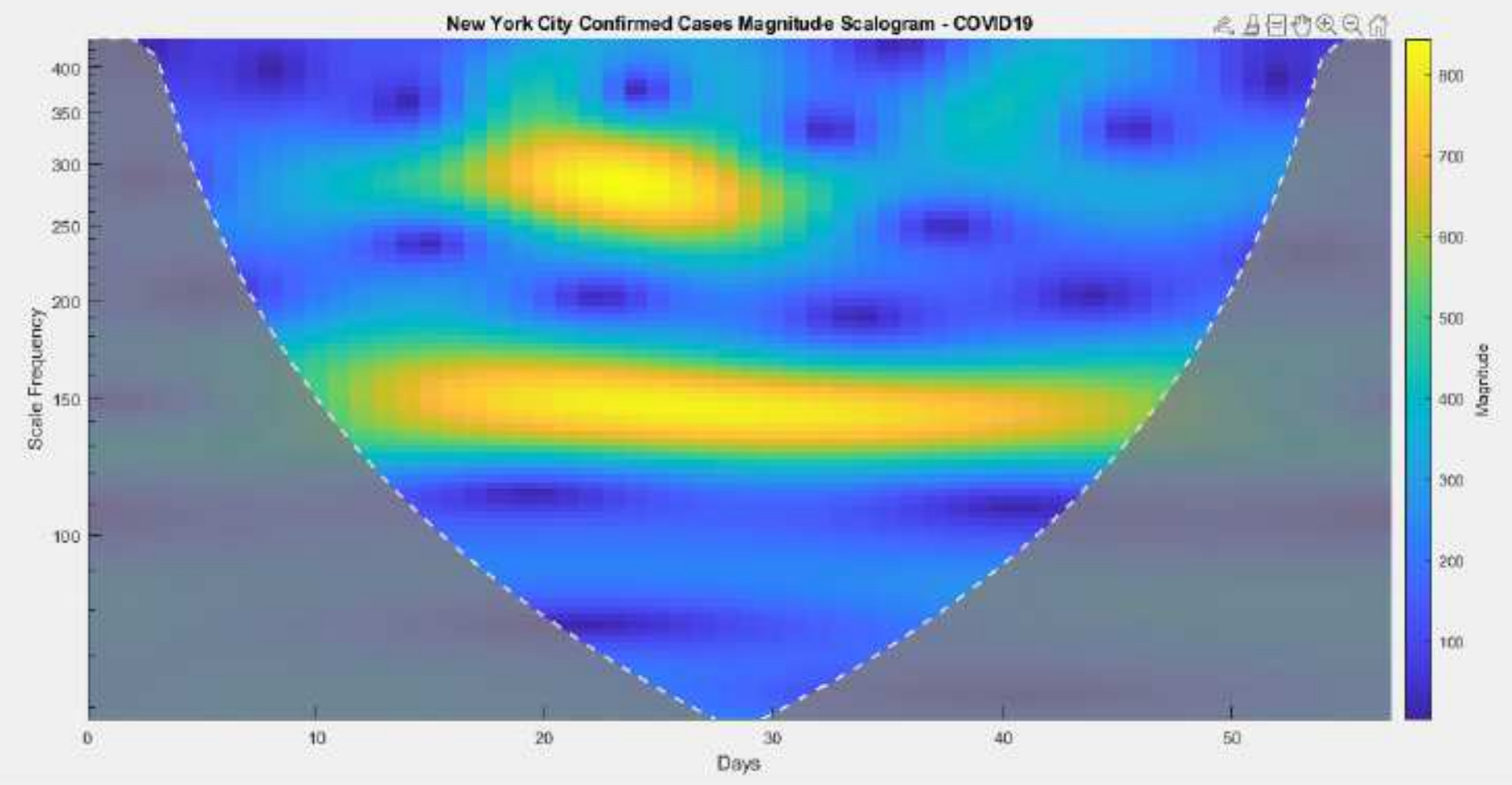

Figure 30

COVID19 - New York City Daily Infected Cases Wavelet Magnitude Scalogram, the date is from 3/9/2020 to $5 / 5 / 2020$

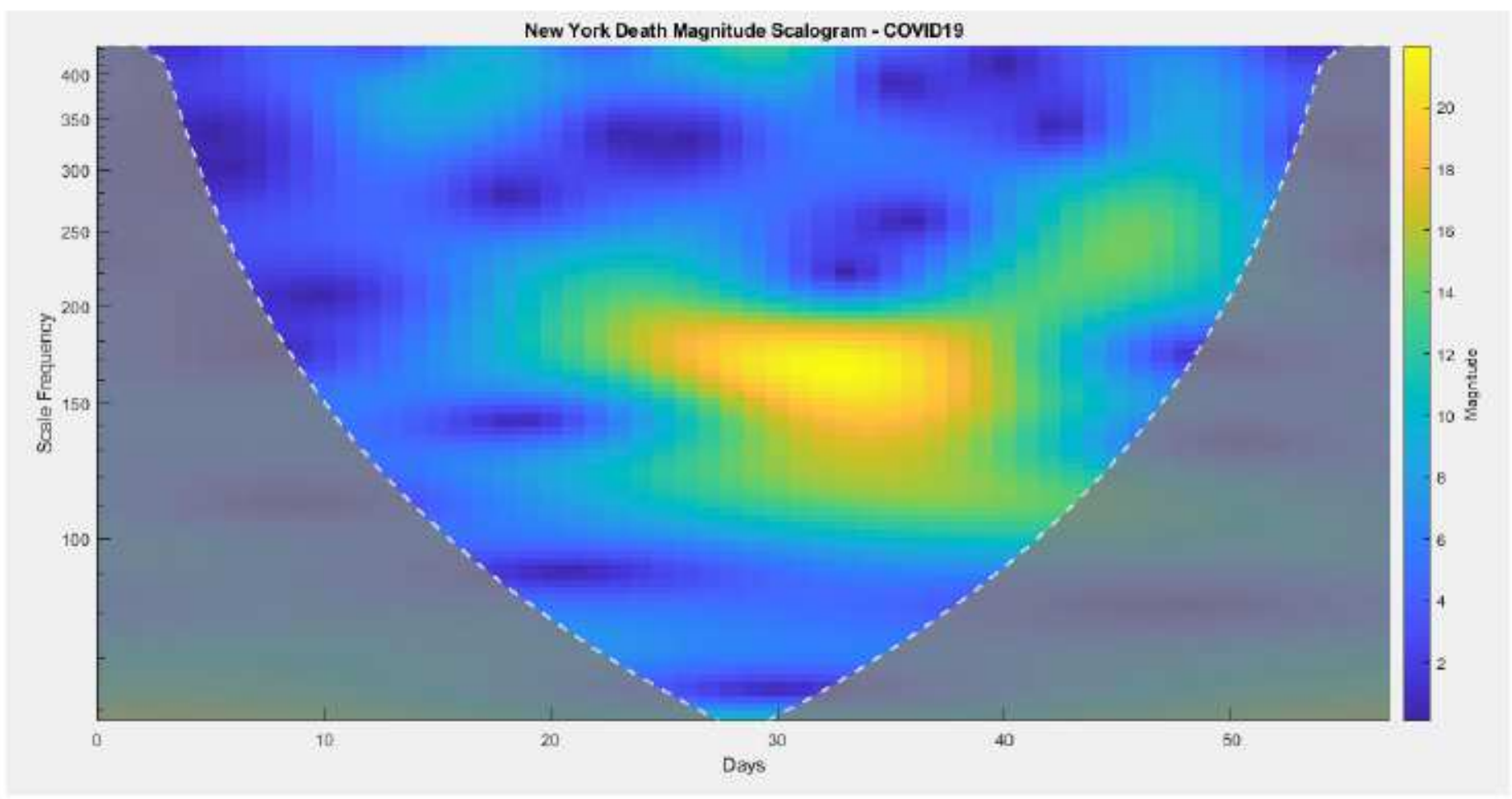


Figure 31

COVID 19 - New York City Daily Deaths Wavelet Magnitude Scalogram, the date is from 3/9/2020 to $5 / 5 / 2020$. 